\title{
Cosmic ray feedback in hydrodynamical simulations of galaxy formation
}

\author{
M. Jubelgas ${ }^{1}$, V. Springel ${ }^{1}$, T. Enßlin ${ }^{1}$, and C. Pfrommer ${ }^{1,2}$ \\ 1 Max-Planck-Institut für Astrophysik, Karl-Schwarzschild-Straße 1, 85740 Garching bei München, Germany \\ e-mail: [jubelgas;vspringel; ensslin] @mpa-garching.mpg.de \\ 2 Canadian Institute for Theoretical Astrophysics, University of Toronto, 60 St. George Street, Toronto, Ontario, M5S 3H8, Canada \\ e-mail: pfrommer@cita.utoronto.ca
}

Received 28 March 2006 / Accepted 16 November 2007

\begin{abstract}
It is well known that cosmic rays contribute significantly to the pressure of the interstellar medium in our own Galaxy, suggesting that they may play an important role in regulating star formation during the formation and evolution of galaxies. We here discuss a novel numerical treatment of the physics of cosmic rays and its implementation in the parallel smoothed particle hydrodynamics code GADGET-2. In our methodology, the non-thermal cosmic ray population of each gaseous fluid element is approximated by a simple power law spectrum in particle momentum, characterized by an amplitude, a cut-off, and a fixed slope. Adiabatic compression and a number of physical source and sink terms are modelled which modify the cosmic ray pressure of each particle. The most important sources considered are injection by supernovae and diffusive shock acceleration, while the primary sinks are thermalization by Coulomb interactions, and catastrophic losses by hadronic interactions. We also include diffusion of cosmic rays. Using a number of test problems, we show that our scheme is numerically robust and efficient, allowing us to carry out the first cosmological structure formation simulations that account for cosmic ray physics, together with radiative cooling and star formation. In simulations of isolated galaxies, we find that cosmic rays can significantly reduce the star formation efficiencies of small galaxies, with virial velocities below $\sim 80 \mathrm{~km} \mathrm{~s}^{-1}$, an effect that becomes progressively stronger towards low-mass scales. In cosmological simulations of the formation of dwarf galaxies at high redshift, we find that the total mass-to-light ratio of small halos and the faint end of the luminosity function are affected. The latter becomes slightly flatter. When cosmic ray acceleration in shock waves is followed as well, we find that up to $40 \%$ of the energy dissipated at structure formation shocks can appear as cosmic ray pressure at redshifts around $z \sim 3-6$, but this fraction drops to $\sim 10 \%$ at low redshifts when the shock distribution becomes increasingly dominated by lower Mach numbers. Despite this large cosmic ray energy content in the high-redshift intergalactic medium, the flux power spectrum of the Lyman- $\alpha$ forest is only affected on very small scales of $k>0.1 \mathrm{~km}^{-1} \mathrm{~s}$, and at a weak level of 5-15\%. Within virialized objects, we find lower contributions of CR-pressure, due to the increased efficiency of loss processes at higher densities, the lower Mach numbers of shocks inside halos, and the softer adiabatic index of CRs, which disadvantages them when a composite of thermal gas and cosmic rays is adiabatically compressed. The total energy in cosmic rays relative to the thermal energy within the virial radius drops from $20 \%$ for $10^{12} h^{-1} M_{\odot}$ halos to $5 \%$ for rich galaxy clusters of mass $10^{15} \mathrm{~h}^{-1} M_{\odot}$ in non-radiative simulations. Interestingly, the lower effective adiabatic index also increases the compressibility of the intrahalo medium, an effect that slightly increases the central concentration of the gas and the baryon fraction within the virial radius. We find that this can enhance the cooling rate onto central cluster galaxies, even though the galaxies in the cluster periphery become slightly less luminous as a result of cosmic ray feedback.
\end{abstract}

Key words. methods: numerical - acceleration of particles - ISM: general - galaxies: structure - galaxies: clusters: general intergalactic medium

\section{Introduction}

In recent years, the $\Lambda \mathrm{CDM}$ model has emerged as a highly successful "concordance" model for cosmological structure formation. It conjectures that the dominant mass component in the universe consists of cold dark matter and that a cosmological constant or dark energy field adds sufficient energy density to yield a spatially flat spacetime. This model is impressively successful in matching observational data on a wide range of scales and epochs, including the cosmic microwave background fluctuations (e.g. Spergel et al. 2003), galaxy clustering (e.g. Tegmark et al. 2004; Cole et al. 2005), cosmic flows in the present universe (e.g. Willick et al. 1997; Hudson et al. 2004), or the observational data on distant supernovae (Riess et al. 1998; Perlmutter et al. 1999).

While the dynamics of the dark matter component in the $\Lambda \mathrm{CDM}$ model is now quite well understood and can be followed with high accuracy in numerical simulations (Power et al. 2003; Navarro et al. 2004; Heitmann et al. 2005), the baryonic processes that regulate the formation of the luminous components of galaxies are much less well understood. Direct hydrodynamical simulations that follow the baryonic gas as well as the dark matter, face a number of "small-scale" problems. For example, they tend to produce too many stars as a result of a "cooling catastrophe", unless effects like galactic outflows are included in a phenomenological way (e.g. Springel \& Hernquist 2003b). They lead to disk galaxies that are too concentrated (e.g. Abadi et al. 2003) and fail to reproduce the observed shape of the luminosity function of galaxies in detail (Murali et al. 2002; Nagamine et al. 2004).

By invoking strong feedback processes, semi-analytic models of galaxy formation are able to overcome these problems and to explain a wide array of galaxy properties (White \& Frenk 1991; Kauffmann et al. 1993; Baugh et al. 1998; 
Somerville \& Primack 1999; Cole et al. 2000; Croton et al. 2006). While this supports the notion that feedback is crucial for the regulation of galaxy formation, it is unclear whether the physical nature of the feedback processes is correctly identified in the present semi-analytic models, or whether they merely give a more or less correct account of the consequences of this feedback. Direct hydrodynamic simulations can in principle be used to lift this ambiguity and to more directly constrain the physical processes at work.

In most current models of galaxy formation, feedback effects due to supernovae explosions and due to a photoionizing background are usually included, and more recently, some studies have considered quasar and radio activity by AGN as well (e.g. Di Matteo et al. 2005; Sijacki \& Springel 2006). However, perhaps surprisingly, magnetic fields and non-thermal pressure components from cosmic rays have received comparatively little attention thus far (with notable exceptions, including Kang et al. 1996; Miniati 2001; Miniati et al. 2001; Miniati 2002, 2003; Ryu \& Kang 2003, 2004), despite the fact that cosmic rays are known to contribute substantially to the pressure in the ISM of our own Galaxy. This is probably at least in part due to the complexity of the cosmic ray dynamics, which when coupled to the galaxy formation process is very hard to describe analytically. Even when numerical methods are invoked, the cosmic ray physics is so involved that a number of simplifying approximations are required to make it tractable in a cosmological simulation settings.

In recent years, a number of numerical studies have addressed this challenge, and provided very interesting results that suggest an important role of cosmic ray production for the $\gamma$-ray background and the structure of clusters of galaxies. Indeed, Miniati et al. (2001) simulated cosmic ray production at structure formation shock waves and found that up to $\sim 45 \%$ of the pressure in groups and clusters of galaxies could be due to cosmic rays. Similar results were also found by Ryu \& Kang (2003) who reported that the cosmic ray energy in the cluster ICM could account for up to half of the thermal energy. However, so far these numerical studies have focused on the acceleration process in structure formation shocks. While some studies accounted for CR transport and loss processes (e.g. Miniati 2001), they typically neglected radiative cooling and star formation that are important in forming individual galaxies.

In this study, our goal is to introduce the first cosmological code of galaxy formation that treats cosmic rays during the structure formation process. Our principal approach for capturing the cosmic ray physics has been laid out in a companion paper (Enßlin et al. 2007), where we introduced a number of approximations to reduce the complexity of the problem. Fundamentally, we model the cosmic ray population of each fluid element with a power law spectrum in particle momentum, characterized by an amplitude, a cut-off, and a fixed slope. Our model then accounts for adiabatic advection of cosmic rays, and for injection and loss terms due to a variety of physical sources. Finally, we also include cosmic ray diffusion. The primary injection mechanisms we consider are supernova shocks and diffusive shock acceleration at structure formation shock waves. Since the efficiency of the latter is a sensitive function of the Mach number of the shock, we have also developed an on-the-fly shock finder for SPH calculations, which is described in a second companion paper (Pfrommer et al. 2006).

In this paper, we use the theoretical model of Enßlin et al. (2007) and cast it into a numerical formulation of cosmic ray physics that we implement in the cosmological TreeSPH code GADGET-2 (Springel et al. 2001; Springel 2005). We discuss our numerical approach in detail, including also various

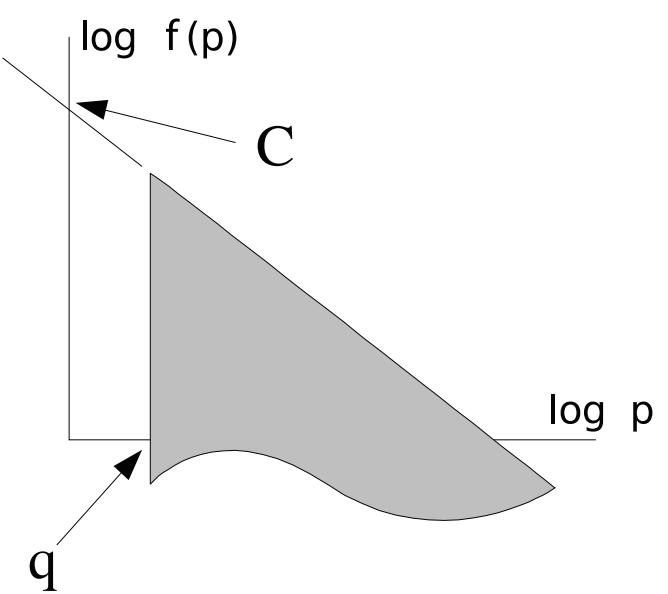

Fig. 1. Schematic illustration of the cosmic ray momentum spectrum in our two parameter model. We adopt a simple power-law description, where the slope of the cosmic ray spectrum is given by a spectral in$\operatorname{dex} \alpha$, kept constant throughout our simulation. The normalization of the spectrum is given by the variable $C$, and the low-momentum cutoff is expressed in terms of a dimensionless variable $q$, in units of $m_{\mathrm{p}} c$ where $m_{\mathrm{p}}$ is the proton rest mass.

optimisations needed to keep the scheme efficient and robust. We then move on to show first results from applications of the model, ranging from isolated galaxies of different sizes, to cosmological simulations of galaxy cluster formation, and of homogeneously sampled boxes. Interestingly, cosmic rays can have a substantial effect on dwarf galaxies, suppressing their star formation considerably. We show that this should leave a noticeable imprint in the luminosity function of galaxies, leading to a shallower faint-end slope.

This paper is laid out as follows. In Sect. 2, we describe the details of our implementation of cosmic ray physics, and in Sect. 3 we discuss our treatment of cosmic ray diffusion. Section 4 presents a number of test problems, which we used to verify the validity of results obtained by the code. We then describe in Sect. 5 a first set of simulations of isolated galaxies carried out with the new code. This establishes a number of principal effects found for the model. In Sect. 6, we then extend our analysis to more sophisticated, fully cosmological simulations of structure formation. We consider both galaxy clusters and dwarf galaxy formation at high redshift. Finally, Sect. 7 summarizes our conclusions and gives an outlook for future studies of cosmic rays in a cosmological context.

\section{Modelling cosmic ray physics}

In Enßlin et al. (2007), we have introduce a new theoretical formalism for a simplified treatment of cosmic ray physics during cosmological structure formation. We also gave a detailed discussion of the physical background and the relative importance of various physical source and sink processes, and how they can be incorporated within the simplified framework. In this section of the present study, we describe the practical implementation of this model within the Lagrangian TreeSPH code GADGET-2, including also a concise summary of the those parts of the framework of Enßlin et al. (2007) that we included in the code thus far.

\subsection{The cosmic ray spectrum and its adiabatic evolution}

As discussed in full detail in Enßlin et al. (2007), we assume that the cosmic ray population of each fluid element is made up 


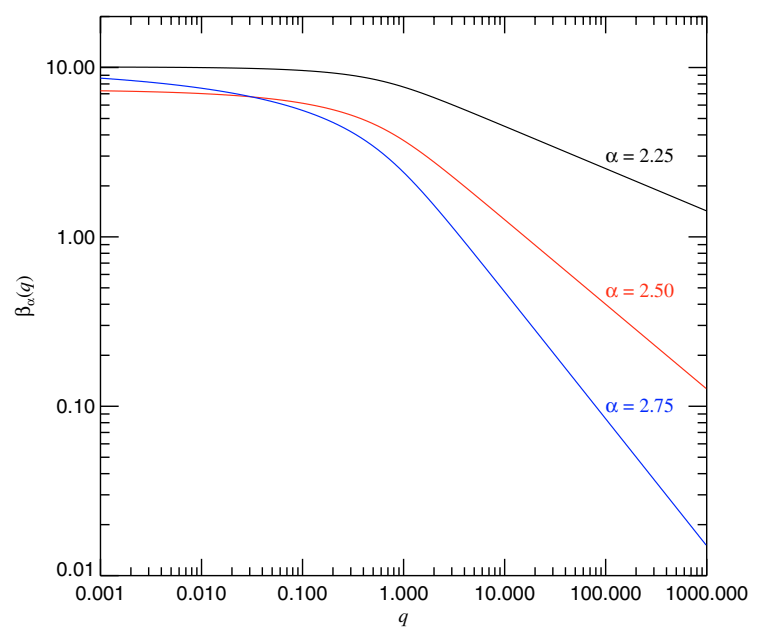

Fig. 2. The function $\beta_{\alpha}(q)$ introduced in Eq. (5), for several different values of the spectral slope $\alpha$.

of relativistic protons with an isotropic momentum distribution function of the form

$\frac{\mathrm{d}^{2} N}{\mathrm{~d} p \mathrm{~d} V}=C p^{-\alpha} \theta(p-q)$,

where $C$ gives the normalization, $q$ is a low momentum cut-off, and $\alpha$ is the power law slope. The momenta are expressed in dimensionless form in units of $m_{\mathrm{p}} c$, where $m_{\mathrm{p}}$ is the proton mass. For the purposes of this paper, we will generally take $\alpha$ to be constant $(\alpha \sim 2.5-2.8)$, which should be a reasonable first order approximation in most cases relevant to galactic structure formation. We note however that $\alpha$ can in principle be made to vary in our formalism, at the price of a substantially increased computational cost and complexity (Enßlin et al. 2007). The pressure of this cosmic ray population is given by

$P_{\mathrm{CR}}=\frac{C m_{\mathrm{p}} c^{2}}{6} \mathcal{B}_{\frac{1}{1+q^{2}}}\left(\frac{\alpha-2}{2}, \frac{3-\alpha}{2}\right)$,

while the number density is simply $n_{\mathrm{CR}}=C q^{1-\alpha} /(\alpha-1)$. Here

$\mathcal{B}_{n}(a, b) \equiv \int_{0}^{n} x^{a-1}(1-x)^{b-1} \mathrm{~d} x$

denotes incomplete Beta functions. To describe the kinetic energy per cosmic ray particle for such a power-law population we define the function

$T_{\mathrm{CR}}(\alpha, q) \equiv\left[\frac{1}{2} q^{\alpha-1} \beta_{\alpha}(q)+\sqrt{1+q^{2}}-1\right] m_{\mathrm{p}} c^{2}$,

which will be of later use. The quantity

$\beta_{\alpha}(q) \equiv \mathcal{B}_{\frac{1}{1+q^{2}}}\left(\frac{\alpha-2}{2}, \frac{3-\alpha}{2}\right)$

is here introduced as a convenient abbreviation for the incomplete Beta function. We show $\beta_{\alpha}(q)$ as a function of $q$ for a few values of $\alpha$ in Fig. 2.

We here implement the cosmic ray model in a Lagrangian simulation code, where the advection of the cosmic ray population can be conveniently described simply in terms of the motion of gas particles. In this approach, the normalization of the spectrum should be expressed in terms of a quantity normalized to mass, instead of the volume-normalized quantity $C$, with the translation between the two being simply given by the local gas density $\rho$. In our case, it is convenient to absorb the proton mass into a redefinition of the amplitude, so we define

$\tilde{C}=C \frac{m_{\mathrm{p}}}{\rho}$

as Lagrangian amplitude of the spectrum. Upon adiabatic changes of the gas density, the normalization of the spectrum changes according to

$\tilde{C}(\rho)=\left(\frac{\rho}{\rho_{0}}\right)^{\frac{\alpha-1}{3}} \tilde{C}_{0}$,

while the momentum cut-off shifts as

$q(\rho)=\left(\frac{\rho}{\rho_{0}}\right)^{\frac{1}{3}} q_{0}$.

Here, we introduced a reference density $\rho_{0}$ (for example set equal to the mean cosmic density) and a corresponding normalization $\tilde{C}_{0}$ and cut-off $q_{0}$ at this density. In our numerical implementation, we only have to follow the evolution of the adiabatic invariants $\tilde{C}_{0}$ and $q_{0}$ due to non-trivial physical source and sink processes, releasing us from the task to compute adiabatic changes of the normalization and cut-off explicitly. Instead, they are simply accounted for by Eqs. (7) and (8).

The number density $n_{\mathrm{CR}}$ of relativistic CR protons can also be conveniently expressed in terms of the total proton density ${ }^{1}$, yielding

$\tilde{n}=n_{\mathrm{CR}} \frac{m_{\mathrm{p}}}{\rho}=\tilde{C} \frac{q^{1-\alpha}}{\alpha-1}=\tilde{C}_{0} \frac{q_{0}^{1-\alpha}}{\alpha-1}$.

We can thus interpret $\tilde{n}$ as something like a "cosmic ray to baryon ratio". This quantity is an adiabatic invariant which can be followed accurately in dynamical simulations with our Lagrangian approach.

Note that in our model we do not explicitely remove baryons from the reservoir of ordinary thermal matter when they are accelerated to become relativistic cosmic ray particles. This is a valid approximation, provided we have $\tilde{n} \ll 1$, which is always expected in our applications. In the calculations we performed so far, the fraction of protons contained in the relativistic phase typically remained far below a maximum value of $\tilde{n} \approx 10^{-4}$. The latter is already an exceptionally large value which we encountered only in our most extreme tests, but it is still small enough that the reduction of the number density of thermal particles can be safely neglected. We note that cosmic ray confinement by magnetic fields holds in ideal magneto-hydrodynamics (MHD) when the mass fraction in relativistic particles is small.

In a Lagrangian code, it is natural to express the cosmic ray energy content in terms of energy per unit gas mass, $\tilde{\varepsilon}$, which is given by

$\tilde{\varepsilon}=c^{2} \frac{\tilde{C}}{\alpha-1}\left[\frac{1}{2} \beta_{\alpha}(q)+q^{1-\alpha}\left(\sqrt{1+q^{2}}-1\right)\right]$.

Note that $\tilde{\varepsilon}$ refers to the energy normalized by the total gas mass, not by the mass of the cosmic ray particles alone. The specific energy content can also be expressed as

$\tilde{\varepsilon}=\frac{T_{\mathrm{CR}} n_{\mathrm{CR}}}{\rho}=\frac{T_{\mathrm{CR}} \tilde{n}}{m_{\mathrm{p}}}$.

1 We here loosely call $\rho / m_{\mathrm{p}}$ the proton density. In our cosmological applications, we of course use the mean particle mass where appropriate to account for the presence of heavier elements and the ionization state of the gas. 


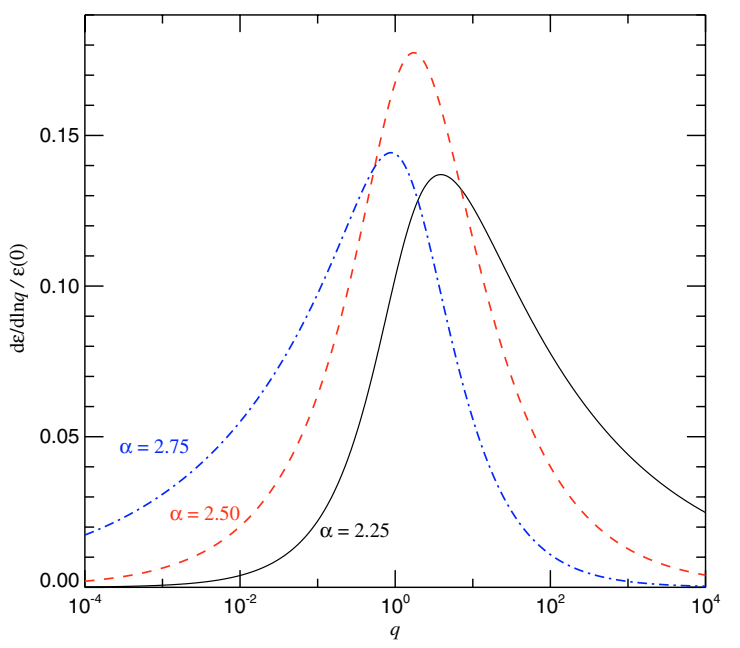

Fig. 3. The distribution of cosmic ray energy per unit logarithmic interval of proton momentum, for several different values of the spectral slope $\alpha$. The distributions have been normalized to $\tilde{\varepsilon}(0)$ in each case.

In Fig. 3, we show the distribution $\mathrm{d} \tilde{\varepsilon} / \mathrm{d} \ln q$ of energy per logarithmic momentum interval, normalized to a spectrum with vanishingly small cut-off. For spectral indices in the range $2<$ $\alpha<3$, most of the energy is typically contained around $q \simeq 1$, unless the cut-off of the actual spectrum lies higher than that, in which case the particles just above the cut-off will dominate the total energy. Due to our assumption that the momentum distribution extends as a power-law to infinity, the spectral index $\alpha$ is restricted to $\alpha>2$, otherwise the energy would diverge. For $\alpha<3$, the energy stays finite also for an arbitrarily low spectral cut-off.

In our numerical scheme, every baryonic SPH particle carries the adiabatic invariants $q_{0}$ and $\tilde{C}_{0}$ as internal degrees of freedom for the description of the cosmic ray physics. These variables are then used to compute all physical properties of the cosmic ray sector, as required for the force evaluations. For the gas dynamics, we are primarily interested in the effective pressure term due to the relativistic particles, which are confined by the ambient magnetic field. In our set of variables, this is compactly given as

$P_{\mathrm{CR}}=\frac{\tilde{C} \rho c^{2}}{6} \beta_{\alpha}(q)$.

In the calculation of the hydrodynamic accelerations with the Euler equation, we can simply add this partial pressure due to CRs to the ordinary thermal pressure (see Enßlin et al. 2007, for further discussion). This makes the interface with the ordinary hydrodynamical code conveniently small, requiring only a small number of changes at well defined places.

The effective adiabatic index of the cosmic ray pressure component upon local isentropic density changes is

$\gamma_{\mathrm{CR}} \equiv \frac{\partial \log P_{\mathrm{CR}}}{\partial \log \rho}=\frac{\alpha+2}{3}-\frac{2}{3} \frac{q^{3-\alpha}}{\beta_{\alpha}(q) \sqrt{1+q^{2}}}$.

On the other hand, when the pressure is expressed in terms of the cosmic ray energy density, we obtain

$\frac{P_{\mathrm{CR}}}{\rho \tilde{\varepsilon}}=\frac{(\alpha-1) \beta_{\alpha}(q)}{3 \beta_{\alpha}(q)+6 q^{1-\alpha}\left(\sqrt{1+q^{2}}-1\right)}$.

In Fig. 4, we show the dependence of the right-hand-side of Eq. (14) on the spectral cut-off $q$, for different values of the

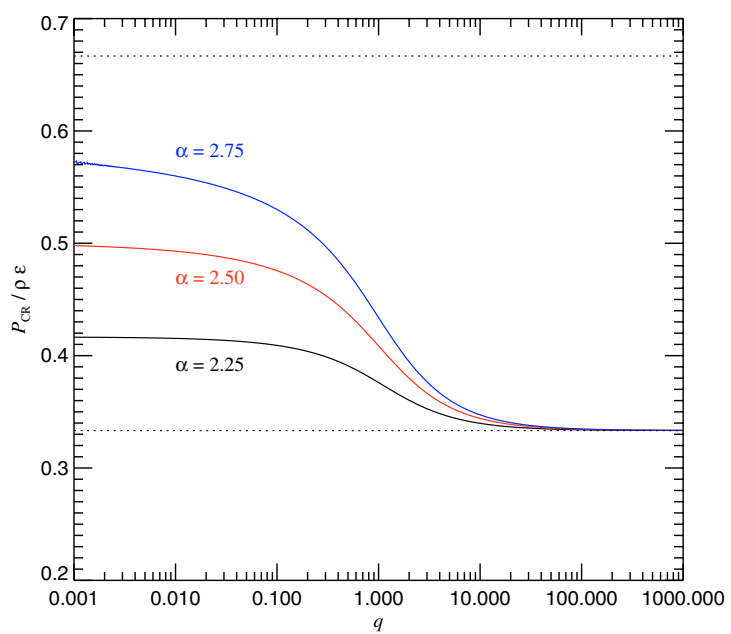

Fig. 4. Cosmic ray pressure in units of the cosmic ray energy density, as a function of the spectral cut-off $q$. Except in the transition region from non-relativistic to relativistic behaviour, the cosmic ray pressure depends only weakly on $q$. In the ultra-relativistic regime, the ratio approaches $P_{\mathrm{CR}} /(\rho \tilde{\varepsilon}) \simeq(4 / 3-1)$, which is shown as the lower dotted line. The upper dotted line gives the expected value of $(5 / 3-1)$ for an ideal gas. For the same energy content, cosmic rays always contribute less pressure than thermal gas.

slope $\alpha$. For large values of $q$ we obtain $P_{\mathrm{CR}} /(\rho \tilde{\varepsilon}) \simeq(4 / 3-1)$, as expected for particles in the ultra-relativistic regime, while for low values of $q$ the ratio is still significantly below the $(5 / 3-1)$ expected for an ideal gas. However, it is clear that for given cosmic ray energy density, the pressure depends only weakly on the spectral cut-off; the value of $\tilde{\varepsilon}$ is hence much more important for the dynamics than the value of $\alpha$.

\subsection{Including non-adiabatic $C R$ processes}

The adiabatic behaviour of cosmic rays that are locally locked into the fluid by magnetic fields can be well traced with the above prescriptions. However, there are also a multitude of physical processes that affect the CR spectrum of a gaseous masselement in a non-adiabatic fashion. For instance, particles can be accelerated in strong shock waves to relativistic momenta and become cosmic rays. This process of diffusive shock acceleration should be particularly effective in high Mach number accretion shocks during cosmological structure formation, which can be traced by the hydrodynamical solver of our simulation code. On sub-resolution scales, violent shocks due to supernova explosions associated with stellar evolution may inject cosmic rays as well. Other astrophysical sources include the ejection of highenergy particles in a jet from an accreting black hole.

On the other hand, the cosmic ray population suffers a number of loss processes which will diminish the abundance over time if there is no new supply of freshly injected or accelerated protons. We shall here consider only the most prominent loss processes in the form of Coloumb losses that thermalize the cosmic ray energy, catastrophic losses that let the energy escape as radiation, and diffusion which washes out cosmic ray pressure gradients.

As discussed above and in Enßlin et al. (2007), our cosmic ray model requires three parameters to describe the state of the relativistic particle component of the gas. One parameter is the spectral index $\alpha$, which we set to a constant value throughout the simulation volume, specified at the start of the simulation with a value motivated by the typically observed 
index in galactic systems. However, we do not restrict the range of non-adiabatic processes we consider to those with a similar injection index. Rather, we translate the injected cosmic ray properties into changes of amplitude and momentum cut-off within the framework of our simplified, fixed-slope model for the cosmic ray spectrum. This translation is based on basic principles of mass and energy conservation. Despite this considerable simplification, it is clear that the thermodynamic state of CR gas is considerably more complex than that of an ideal gas, where essentially everything is determined by the specific entropy alone.

Given some changes within a simulation time step of the cosmic ray specific energy $(\mathrm{d} \tilde{\varepsilon})$ and relative $\mathrm{CR}$ density $(\mathrm{d} \tilde{n})$ associated with a fluid element, these changes can be cast into variations of the adiabatic invariants $q_{0}$ and $\tilde{C}_{0}$ of the cosmic ray population using a Jacobian matrix. We then obtain

$\mathrm{d} \tilde{C}_{0}=\left(\frac{\rho}{\rho_{0}}\right)^{-\frac{\alpha-1}{3}} \mathrm{~d} \tilde{C}=\tilde{C}_{0} \frac{m_{\mathrm{p}} \mathrm{d} \tilde{\varepsilon}-T_{\mathrm{p}}(q) \mathrm{d} \tilde{n}}{m_{\mathrm{p}} \tilde{\varepsilon}-T_{\mathrm{p}}(q) \tilde{n}}$

and

$\mathrm{d} q_{0}=\left(\frac{\rho}{\rho_{0}}\right)^{-\frac{1}{3}} \mathrm{~d} q=\frac{q_{0}}{\alpha-1} \frac{m_{\mathrm{p}} \mathrm{d} \tilde{\varepsilon}-T_{\mathrm{CR}} \mathrm{d} \tilde{n}}{m_{\mathrm{p}} \tilde{\varepsilon}-T_{\mathrm{p}}(q) \tilde{n}}$,

where we used the mean kinetic energy per cosmic ray particle, viz. $T_{\mathrm{CR}}=\tilde{\varepsilon} m_{\mathrm{p}} / \tilde{n}$, as given by Eq. (4), and defined

$T_{\mathrm{p}}(q)=\left(\sqrt{1+q^{2}}-1\right) m_{\mathrm{p}} c^{2}$

as the kinetic energy of a proton with normalized momentum $q$. Recall that $T_{\mathrm{CR}}$ only depends on $q$ and $\alpha$, but does not depend on the normalization of the spectrum.

However, this simple and fast translation scheme will only work for sufficiently small changes of the cosmic ray population. In our implementation, we therefore apply Eqs. (15) and (16) only if the relative changes in cosmic ray energy and number density are less than a few percent. Otherwise, new cosmic ray spectral parameters in terms of $\tilde{C}_{0}$ and $q_{0}$ are computed by explicitly solving Eqs. (9) and (10), after applying the principles of energy and particle conservation. While (9) can be easily solved for either $q_{0}$ or $\tilde{C}_{0}$, Eq. (10) for the specific energy needs to be inverted numerically (see also the discussion in Sect. 4.1, and in Enßlin et al. 2007).

Still, a naive application of energy and particle conservation when adding a newly injected CR component to the current spectrum can cause unphysical results if the spectral cutoffs involved are very different. The reason lies in the strong dependence of the cosmic ray loss processes on particle momentum, together with our simplified spectral representation. As we will see, the life-time of cosmic ray particles grows monotonically with particle momentum. This dependence is particularly steep in the non-relativistic regime $\left(\tau_{\text {losses }}(p) \sim p^{3}\right)$, but becomes much shallower and eventually nearly flat in the mildly relativistic and strongly relativistic regimes. Simply injecting a CR component with very low cut-off to one with high cut-off while enforcing total energy and CR particle number conservation will then result in a new composite spectrum where many of the original CR particles are represented as lower momentum particles. Consequently, their cooling times would be artificially reduced. Ultimately, this problem arises because the number density of the injected particles is dominated by low momenta, and these have cooling times much shorter than any relevant dynamical timescale. If this is the case, then it would make more sense to never inject this population to begin with, and to rather thermalize it instantly, thereby avoiding an unphysical distortion of the composite spectrum.

The two injection processes we consider in this paper (shocks and supernova) both supply power-law distributions of cosmic ray particles which start at very low thermal momenta. For them, we define an injection cut-off $q_{\text {inj }}$ such that only the particles and the energy above the cut-off are injected, while the rest of the energy is instantly thermalized and added to the thermal reservoir. The criterion we choose for defining $q_{\text {inj }}$ is

$\tau_{\text {losses }}\left(q_{\text {inj }}\right)=\tau_{\text {inj }}\left(\tilde{\varepsilon}, q_{\text {inj }}\right)$

where $\tau_{\text {losses }}(q)$ is the cooling timescale

$\tau_{\text {losses }}(q) \equiv \frac{\tilde{\varepsilon}}{|\mathrm{d} \tilde{\varepsilon} / \mathrm{d} t|_{\text {losses }}}$

due to cosmic ray loss processes for a spectrum with cut-off at $q$ and spectral slope $\alpha$. As we will see, this timescale is independent of the normalization of the spectrum, and inversely proportional to the density, provided the weak density-dependence of the Coulomb logarithm in the Coulomb loss-rate is neglected. Given the present cosmic ray energy content $\tilde{\varepsilon}$, the timescale

$\tau_{\text {inj }}\left(\tilde{\varepsilon}, q_{\text {inj }}\right) \equiv \frac{\tilde{\varepsilon}}{f_{\alpha_{\text {inj }}}\left(q_{0}, q_{\text {inj }}\right)(\mathrm{d} \tilde{\varepsilon} / \mathrm{d} t)_{\text {inj }}}$

defines the current heating time due to the injection source, assuming that only the fraction $f\left(q_{0}, q_{\text {inj }}\right)$ above $q_{\text {inj }}$ of the raw energy input rate $(\mathrm{d} \tilde{\varepsilon} / \mathrm{d} t)_{\mathrm{inj}}$ contributes efficiently to the build up of the cosmic ray population. Here $q_{0}$ is the intrinsic injection cut-off of the source, which typically lies at very small thermal momenta, and $\alpha_{\text {inj }}$ is the slope of the source process. The factor $f_{\alpha_{\text {inj }}}\left(q_{0}, q_{\text {inj }}\right)$ is given by

$f_{\alpha_{\text {inj }}}\left(q_{0}, q_{\text {inj }}\right)=\left(\frac{q_{\text {inj }}}{q_{0}}\right)^{1-\alpha_{\text {inj }}} \frac{T_{\mathrm{CR}}\left(\alpha_{\text {inj }}, q_{\text {inj }}\right)}{T_{\mathrm{CR}}\left(\alpha_{\text {inj }}, q_{0}\right)}$

for $q_{\text {inj }} \geq q_{0}$, otherwise by unity.

Equation (18) simply says that we only inject cosmic ray particles at momenta where a spectral component could grow, given the rate of energy injection. It would be unphysical to assume that a spectrum develops that extends as a power-law to momenta lower than $q_{\text {inj. }}$. The addition of this injection rule is hence necessary to make our simple model with a fixed spectral shape physically well-behaved.

We note that Eq. (18) will typically have two solutions, or may not have a solution at all if the current energy $\tilde{\varepsilon}$ in cosmic rays is high enough. In the former case, the physical solution is the smaller of the two, which lies at $q_{\text {inj }} \leq q_{\max }$, where $q_{\max }$ is the place where the expression $\tau_{\text {losses }}\left(q_{\text {inj }}\right) f\left(q_{0}, q_{\text {inj }}\right)$ attains its maximum, i.e.

$$
\left.\frac{\mathrm{d}}{\mathrm{d} q}\left[\tau_{\text {losses }}(q) f\left(q_{0}, q\right)\right]\right|_{q=q_{\max }}=0 .
$$

In the latter case, we choose $q_{\text {inj }}=q_{\text {max }}$, which comes closest to solving Eq. (18) and naturally corresponds to the point where one expects the largest amount of cosmic ray energy that can be present as a power-law with a balance between loss and source processes. 


\subsection{Shock acceleration}

In our present model, we consider two primary sources for cosmic rays, diffusive shock acceleration and supernovae. In the former, a small fraction of the particles streaming through a shock front is assumed to diffuse back and forth over the shock interface, experiencing multiple accelerations. This can only happen to particles in the high-energy tail of the energy distribution, and eventually results in a power-law momentum distribution function for the accelerated particles.

In the linear regime of $\mathrm{CR}$ acceleration, particles above a threshold momentum $q_{\text {inj }}$ can be accelerated. They are redistributed into a power-law distribution in momentum that smoothly joins the Maxwell-Boltzmann distribution of the thermal post-shock gas. The slope of the injected CR spectrum is given by

$\alpha_{\text {inj }}=\frac{r+2}{r-1}$,

where $r=\rho_{2} / \rho_{1}$ is the density compression ratio at the shock (Bell 1978a,b). If the shock is dominated by the thermal pressure, the spectral index can also be expressed through the Mach number $M$ as

$\alpha_{\text {inj }}=\frac{4-M^{2}+3 \gamma M^{2}}{2\left(M^{2}-1\right)}$.

The stronger the shock becomes, the flatter the spectrum of the accelerated CR particles, and hence the more high-energy particles are produced. Weak shocks on the other hand produce only rather steep spectra where most of the particles thermalize quickly.

Due to the continuity between the power law and the thermal spectrum, the injected cosmic ray spectrum is completely specified by the injection threshold $q_{0}$, provided the shock strength is known (Miniati 2001, and references therein). We will assume that $q_{0}$ is at a fixed multitude $x_{\text {inj }}$ of the average thermal post-shock momentum, $p_{\text {th }}=\sqrt{2 k T_{2} /\left(m_{\mathrm{p}} c^{2}\right)}$, i.e. $q_{0}=x_{\text {inj }} p_{\text {th }}$. In this case, the fraction of particles that experience shock acceleration does not depend on the post-shock temperature $T_{2}$, and is given by

$\Delta \tilde{n}_{\text {lin }}=\frac{4}{\sqrt{\pi}} \frac{x_{\text {inj }}^{3}}{\alpha_{\text {inj }}-1} \mathrm{e}^{-x_{\text {inj }}^{2}}$.

We will typically adopt a fixed value of $x_{\text {inj }} \simeq 3.5$, motivated by theoretical studies of shocks in galactic supernova remnants (e.g. Drury et al. 1989). The fraction of injected supernovae particles in strong shocks is then a few times $10^{-4}$ (Drury et al. 1989; Jones \& Kang 1993; Kang \& Jones 1995).

In the linear regime of $\mathrm{CR}$ acceleration, the specific energy per unit gas mass in the injected cosmic ray population is given by

$\Delta \tilde{\varepsilon}_{\text {lin }}=\frac{T_{\mathrm{CR}}\left(\alpha_{\text {inj }}, q_{\text {inj }}\right) \Delta \tilde{n}_{\text {lin }}}{m_{\mathrm{p}}}$.

We can use this value to define a shock injection efficiency for CRs by relating the injected energy to the dissipated energy per unit mass at the shock front. The latter appears as extra thermal energy above the adiabatic compression at the shock and is given by $\Delta u_{\text {diss }}=u_{2}-u_{1} r^{\gamma-1}$, where $u_{1}$ and $u_{2}$ are the thermal energies per unit mass before and after the shock, respectively. The injection efficiency of linear theory is then given by

$\zeta_{\text {lin }} \equiv \frac{\Delta \tilde{\varepsilon}_{\text {lin }}}{\Delta u_{\text {diss }}}$.
However, the shock acceleration effect experiences saturation when the dynamical CR becomes comparable to the upstream ram pressure $\rho_{1} v_{1}^{2}$ of the flow. We account for this by adopting the limiter suggested by Enßlin et al. (2007), and define as final acceleration efficiency

$\zeta_{\text {inj }}=\left[1-\exp \left(-\frac{\zeta_{\text {lin }}}{\zeta_{\max }}\right)\right] \zeta_{\max }$.

We will adopt $\zeta_{\max }=0.5$ for the results of this study. Thus, we take the injected energy to be

$\Delta \tilde{\varepsilon}_{\text {inj }}=\zeta_{\text {inj }} \Delta u_{\text {diss }}$,

and correspondingly, the injected particle number is given by

$\Delta \tilde{n}_{\mathrm{inj}}=\frac{m_{\mathrm{p}} \Delta \tilde{\varepsilon}_{\mathrm{inj}}}{T_{\mathrm{CR}}\left(\alpha_{\mathrm{inj}}, q_{0}\right)}$,

where $T_{\mathrm{CR}}\left(\alpha_{\mathrm{inj}}, q_{0}\right)$ is the mean kinetic energy of the accelerated cosmic ray particles. In practice, both $\Delta \tilde{\varepsilon}_{\text {inj }}$ and $\Delta \tilde{n}_{\text {inj }}$ will be lowered when we shift the actual injection point from $q_{0}$ to $q_{\text {inj }}$, as determined by Eq. (18), with the difference of the energies fed to the thermal reservoir directly.

It is clear that the efficiency of CR particle acceleration depends strongly on the compression ratio, or equivalently on the Mach number of shocks. Interestingly, accretion shocks during cosmological structure formation can be particularly strong. Here we hence expect potentially interesting effects both for the forming intragroup and intracluster media, as well as for the intergalactic medium. However, in order to accurately account for the cosmic ray injection by structure formation shocks, we somehow need to be able to estimate the strength of shocks in SPH simulations. As SPH captures shocks with an artificial viscosity instead of an explicit shock detection scheme, this is a non-trivial problem.

In Pfrommer et al. (2006), our second companion paper to this study, we have proposed a practical solution to this problem and developed a novel method for measuring the Mach number of shocks on the fly during cosmological SPH calculation. The method relies on the entropy formulation for SPH by Springel \& Hernquist (2002), and uses the current rate of entropy injection due to viscosity, together with an approximation for the numerical shock transit time, to estimate the shock Mach number. The scheme works better than one may have expected, and it is in fact capable of producing quite accurate Mach number estimates, even for the case of composite gases with a thermal and a cosmic ray pressure component. In cosmological simulations, the method delivers Mach number statistics which agree well with results obtained with hydrodynamical mesh codes that use explicit Riemann solvers (Ryu et al. 2003; Pfrommer et al. 2006).

Having a reliable Mach number estimator solves an important problem when trying to account for cosmic ray injection by shocks in SPH. Another complication is posed by the shock broadening inherent in SPH, which implies a finite shock transit time for particles, i.e. a SPH particle may require several timesteps before it has passed through a shock and received the full dissipative heating. Note that the number of these steps depends on the timestep criterion employed, and can in principle be made very large for a sufficiently conservative choice of the Courant coefficient. Unlike assumed in the above treatment of diffusive shock acceleration, we hence are not dealing with a discrete injection event, but rather need to formulate the cosmic 
ray acceleration in a "continuous fashion", in parallel to the thermal dissipation, such that the final result does not depend on how many timesteps are taken to resolve a broadened shock front.

Fortunately, the above treatment can be easily adjusted to these conditions. We can simply insert for $\Delta u_{\text {diss }}$ in Eq. (29) the dissipated energy in the current timestep. This quantity is computed in the SPH formalism anyway, and in fact, we know that SPH will integrate $\Delta u_{\text {diss }}$ correctly through the shock profile, independent of the number of steps taken. This is because the correct pre- and post-shock state of the gas are enforced by the conservation laws, which are fulfilled by the conservative SPH code. For the same reason, the Rankine-Hugoniot jump conditions are reproduced across the broadened shock. Note however that for computing the linear shock injection efficiency according to Eq. (27), we need to continue to use an estimate for the total energy dissipated across the shock, based on the Mach number finder.

For simplified test calculation with the code, we have also implemented an option where we assume a constant injection efficiency of the shock acceleration process, along with a constant spectral index and momentum cut-off parameter. Values for these parameters can then be chosen to represent the energetically most important types of shocks in the environment to be simulated. Such a simplified injection mechanism can then also be used to get an idea about the importance of the Mach-number dependence of the shock acceleration for different environments.

\subsection{Injection of cosmic rays by supernovae}

Strong shock waves associated with supernovae explosions are believed to be one of the most important cosmic ray injection mechanisms in the interstellar medium. However, similar to star formation itself, individual supernovae are far below our resolution limit in cosmological simulations where we need to represent whole galaxies, or more challenging still, sizable parts of the observable universe. We therefore resort to a subresolution treatment for star formation and its regulation by supernovae (Yepes et al. 1997; Springel \& Hernquist 2003a). In this model, the interstellar medium is pictured as a multiphase medium composed of dense, cold clouds, embedded in a tenuous hot phase. The clouds form by thermal instability out of the diffuse medium, and are the sites of star formation. The massive stars of each formed stellar population are assumed to explode instantly, heating the hot phase, and evaporating some of the cold clouds. In this way, a tight self-regulation cycle for star formation in the ISM is established. In simulations where we include star formation, we employ the default parameters of Springel \& Hernquist (2003a), for which stars can form above a threshold gas density $\rho_{\star}=8.55 \times 10^{6} h^{-2} M_{\odot} \mathrm{kpc}^{-3}$, and $10 \%$ of the stellar mass is formed in massive stars that explode as supernovae. With these settings, the model reproduces the observed "Kennicutt Law" (Kennicutt 1989) of star formation in low-redshift disk galaxies.

To model the generation of cosmic rays, we assume that a certain fraction $\zeta_{\mathrm{SN}} \simeq 0.1-0.3$ of the supernova energy appears as a cosmic ray population (Aharonian et al. 2006; Kang \& Jones 2006). The total rate of energy injection by supernovae for a given star formation rate $\dot{\rho}_{\star}$ depends on the IMF. Assuming a Salpeter IMF and that stars above a mass of $8 M_{\odot}$ explode as supernova with a canonical energy release of $10^{51} \mathrm{erg}$, we obtain roughly one supernova per $250 M_{\odot}$ of stellar mass formed, translating to an energy injection rate per unit volume of $\epsilon_{\mathrm{SN}} \dot{\rho}_{\star}$, with
$\epsilon_{\mathrm{SN}}=4 \times 10^{48} \operatorname{erg} M_{\odot}^{-1}$. We then model the CR energy injection per timestep of a gas particle as

$\Delta \tilde{\varepsilon}_{\mathrm{SN}}=\zeta_{\mathrm{SN}} \epsilon_{\mathrm{SN}} \dot{m}_{\star} \Delta t$,

where $\dot{m}_{\star}=\dot{\rho}_{\star} / \rho$ is the particle's star formation rate per unit mass. Note that uncertainties in the IMF are not really important here as we have introduced a free parameter, $\zeta_{\mathrm{SN}}$, to control the amount of energy that is fed into cosmic rays.

For the slope of the injected cosmic ray power-law we assume a plausible value of $\alpha_{\mathrm{SN}}=2.4$ (Aharonian et al. 2004, 2006), and for the cut-off $q_{\mathrm{SN}}$, we can adopt the thermal momentum $q_{\mathrm{SN}}=\sqrt{k T_{\mathrm{SN}} /\left(m_{\mathrm{p}} c^{2}\right)}$ for a fiducial supernova temperature characteristic for the involved shock acceleration. Our choice of $\alpha_{\mathrm{SN}}=2.4$ for the injection slope is motivated by the observed slope of $\sim 2.75$ in the ISM, and the realization that momentum dependent diffusion in a turbulent magnetic field with a Kolmogorov-type spectrum on small scales should steepen the injected spectrum by $p^{-1 / 3}$ in equilibrium. Our results do not depend on the particular choice for $T_{\mathrm{SN}}$, provided $q_{\mathrm{SN}} \ll 1$. The change of the particle number density can then be computed with the mean kinetic energy $T_{\mathrm{CR}}\left(\alpha_{\mathrm{SN}}, q_{\mathrm{SN}}\right)$ of the injected power law. Using Eqs. (4) and (11), this results in

$\Delta \tilde{n}=m_{\mathrm{p}} \frac{\zeta_{\mathrm{SN}} \epsilon_{\mathrm{SN}} \dot{m}_{\star}}{T_{\mathrm{CR}}\left(\alpha_{\mathrm{SN}}, q_{\mathrm{SN}}\right)} \Delta t$

We note that in the formalism of Springel \& Hernquist (2003a), we need to reduce the supernovae energy injected into thermal feedback (and to an optional wind model if used) by the fraction $\zeta_{\mathrm{SN}}$ that we assume powers cosmic ray acceleration. Also, similar to our treatment of shock injection, $\Delta \tilde{n}$ will be lowered in practice when we shift the actual injection point from $q_{\mathrm{SN}}$ to $q_{\text {inj }}$, as determined by Eq. (18). The resulting difference of the energies is fed directly to the thermal reservoir, contributing to the ordinary supernova feedback modelled by the simulation code.

\subsection{Coulomb cooling and catastrophic losses}

Charged particles moving through a plasma will gradually dissipate their kinetic energy and transfer it to the surrounding ions and electrons by Coulomb interactions. The rate of this energy loss depends both on the physical properties of the surrounding medium and on the detailed momentum spectrum of the cosmic ray population. The latter in particular complicates an accurate determination of the Coulomb loss rate.

Since particles with low momenta are most strongly affected by the Coulomb interactions, a qualitative consequence of this effect is that it induces a flattening of the spectrum; the highmomentum tail remains unchanged while the low-momentum cosmic ray particles dissipate their energy effectively to the thermal gas, and eventually drop out of the cosmic ray population altogether.

In our model, we have deliberately abandoned a detailed representation of the cosmic ray spectrum of each fluid element, in favour of the high computational speed and low memory consumption allowed by our simplified spectral model. We even opted to use a globally fixed spectral index, which means that we cannot represent a spectral flattening in detail. However, we can account for the effect of thermalization of the low-momentum particles in a simple and efficient way. To this end, we compute the energy loss by Coulomb-cooling over the entire spectrum, and then shift the low-momentum cutoff of our spectral model such that just the right amount of energy is removed 
in low-momentum particles, keeping the high-momentum part unaffected.

More specifically, we follow Enßlin et al. (2007) and estimate the Coulomb loss rate of the $\mathrm{CR}$ population as

$$
\begin{aligned}
\left(\frac{\mathrm{d} \tilde{\varepsilon}}{\mathrm{d} t}\right)_{\mathrm{C}}= & -\frac{2 \pi \tilde{C} e^{4} n_{\mathrm{e}}}{m_{\mathrm{e}} m_{\mathrm{p}} c}\left[\ln \left(\frac{2 m_{\mathrm{e}} c^{2}\langle\beta p\rangle}{\hbar \omega}\right)\right. \\
& \left.\times \mathcal{B}_{\frac{1}{1+q^{2}}}\left(\frac{\alpha-1}{2},-\frac{\alpha}{2}\right)-\frac{1}{2} \mathcal{B}_{\frac{1}{1+q^{2}}}\left(\frac{\alpha-1}{2},-\frac{\alpha-2}{2}\right)\right] .
\end{aligned}
$$

Here $n_{\mathrm{e}}$ is the electron abundance, $\omega=\sqrt{4 \pi e^{2} n_{\mathrm{e}} / m_{\mathrm{e}}}$ the plasma frequency, and $\langle\beta p\rangle=3 P_{\mathrm{CR}} /\left(\rho \tilde{n} c^{2}\right)$ is a mean value for our assumed spectrum. We also define a cooling timescale due to Coulomb cooling as

$\tau_{\mathrm{C}}(q)=\frac{\tilde{\varepsilon}}{|\mathrm{d} \tilde{\varepsilon} / \mathrm{d} t|_{\mathrm{C}}}$

which depends only on the spectral cut-off $q$, and is inversely proportional to density, modulo a very weak additional logarithmic density-dependence through the plasma frequency. For a given energy loss $\Delta \tilde{\varepsilon}_{C}$ in a timestep based on this rate, we can then estimate the corresponding change in cosmic ray number density as

$\Delta \tilde{n}_{\mathrm{C}}=\Delta \tilde{\varepsilon}_{\mathrm{C}} \frac{m_{\mathrm{p}}}{T_{\mathrm{p}}(q)}$,

i.e. we assume that the particles are removed at the low momentum cut-off $q$. From Eqs. (15) and (16), we can see that this will result in a gradual rise of the spectral cutoff $q$ while the normalization will remain unchanged. The corresponding energy loss $\Delta \tilde{\varepsilon}_{\mathrm{C}}$ is added to the thermal energy in the ordinary gas component, i.e. the Coulomb cooling process leaves the total energy content of the gas unaffected. We note that for large Coulomb cooling rates, we use an implicit solver to compute the new position of the spectral cut-off, leaving the amplitude parameter exactly invariant. This ensures stability even if the cut-off increases substantially in one timestep.

Another class of loss processes for cosmic rays results occurs through inelastic collisions with atoms of the ambient gas, resulting in the hadronic production of pions, which subsequently decay into $\gamma$-rays, secondary electrons, and neutrinos. In this case, the energy is ultimately dissipated into photons which tend to escape. So here the net effect is a loss of energy from the system, unlike in the case of Coulomb losses, where the dissipated cosmic ray energy heats the thermal reservoir.

However, these "catastrophic losses" can only proceed efficiently when the cosmic ray particles exceed the energy threshold of $q_{\mathrm{thr}} m_{\mathrm{p}} c^{2}=0.78 \mathrm{GeV}$ for pion production $\left(q_{\mathrm{thr}}=0.83\right)$. The total loss rate can then be described by (Enßlin et al. 2007)

$$
\left(\frac{\mathrm{d} \tilde{\varepsilon}}{\mathrm{d} t}\right)_{\mathrm{had}}=-\frac{c \rho \bar{\sigma}_{\mathrm{pp}} \tilde{C} q_{\star}^{1-\alpha} T_{\mathrm{CR}}\left(\alpha, q_{\star}\right)}{2(\alpha-1) m_{\mathrm{p}}^{2}},
$$

where $\bar{\sigma}_{\mathrm{pp}} \simeq 32$ mbarn is the averaged pion production cross section, and $q_{\star}$ denotes $q_{\star}=\max \left\{q, q_{\text {thr }}\right\}$. The number density of cosmic ray particles stays constant, however, due to conservation of baryon number in strong and electroweak interactions, i.e. we have $\Delta \tilde{n}_{\text {had }}=0$. This condition in turn implies that the changes of amplitude and cut-off of our spectral model due to this cooling process are related by

$$
\frac{\Delta \tilde{C}}{\tilde{C}}=(\alpha-1) \frac{\Delta q}{q} \text {. }
$$
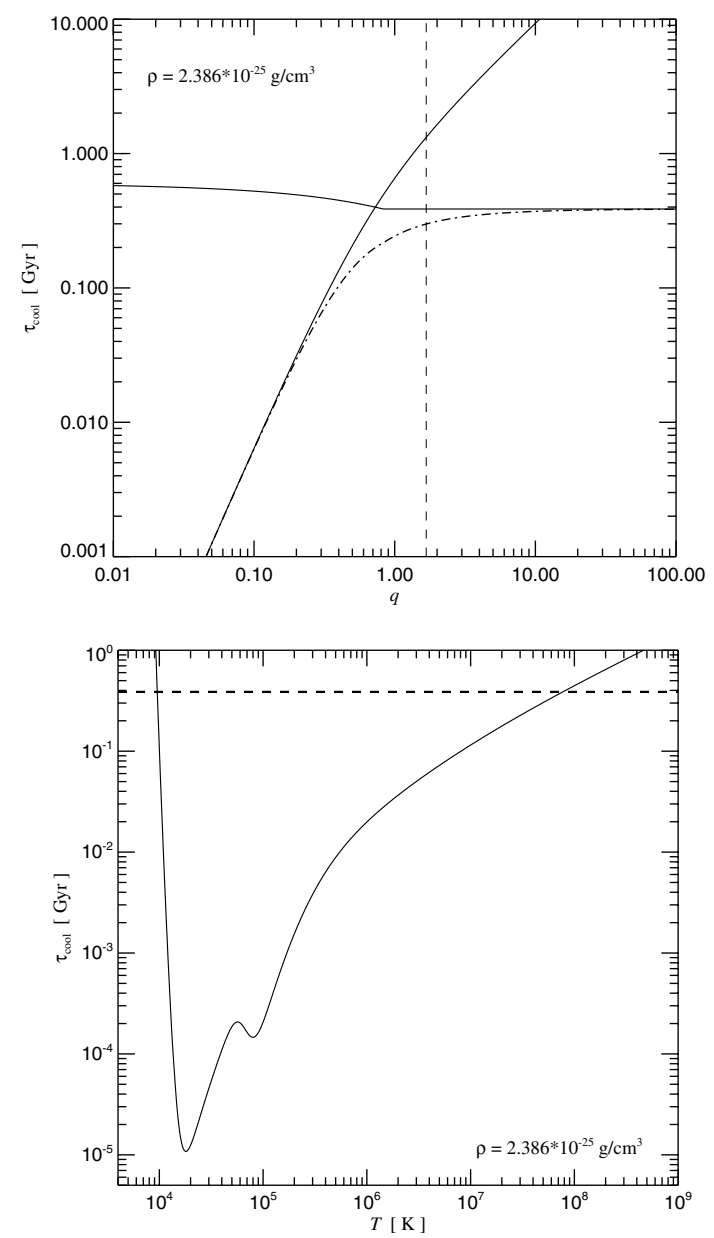

Fig. 5. The top panel shows the cooling times due to Coulomb losses (rising solid line) and hadronic dissipation (nearly horizontal line) as a function of the spectral cut-off. The dot-dashed line gives the total cooling time, while the vertical dashed lines marks the asymptotic equilibrium cut-off reached by the CR spectrum when no sources are present. The bottom panel shows the cooling time of ordinary thermal gas due to radiative cooling (for primordial metallicity), as a function of temperature. The horizontal dashed line marks the cooling time of CRs with a high momentum cut-off ( $q \gg 1)$, for comparison. In both panels, the times have been computed for a gas density of $\rho=2.386 \times$ $10^{-25} \mathrm{~g} \mathrm{~cm}^{-3}$, which corresponds to the density threshold for star formation that we usually adopt. Note however that the cooling times all scale as $\tau \propto 1 / \rho$, i.e. for different densities only the vertical scale would change but the relative position of the lines would remain unaltered.

As before, we also define a cooling timescale due to catastrophic losses, which is given by

$\tau_{\text {had }}(q) \equiv \frac{\tilde{\varepsilon}}{|\mathrm{d} \tilde{\varepsilon} / \mathrm{d} t|_{\text {had }}}=\frac{2 m_{\mathrm{p}}}{c \rho \bar{\sigma}_{\mathrm{pp}}} \frac{T_{\mathrm{CR}}(\alpha, q)}{T_{\mathrm{CR}}\left(\alpha, q_{\star}\right)}\left(\frac{q}{q_{\star}}\right)^{1-\alpha}$

Note that $\tau_{\text {had }}$ becomes constant for $q \geq q_{\mathrm{thr}}$.

In the top panel of Fig. 5, we show the cooling timescales for Coulomb and catastrophic losses as a function of the cutoff parameter $q$, at a fiducial density corresponding to our star formation threshold, assuming a spectral index $\alpha=2.5$. As expected, catastrophic losses dominate for high momentum cutoffs, and therefore limit the lifetime of any cosmic ray population to $\tau \sim 2 m_{\mathrm{p}} /\left(c \rho \bar{\sigma}_{\mathrm{pp}}\right)$, unless an injection process provides a resupply. For small cut-offs, Coulomb losses dominate and will 


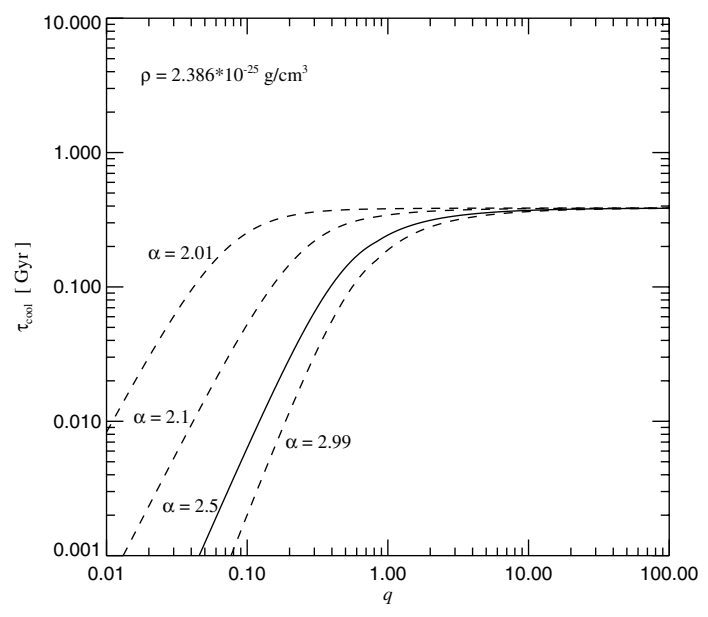

Fig. 6. The total cooling time due to Coulomb losses and hadronic dissipation as a function of the spectral cut-off, for different assumptions about the slope of the spectrum ( $\alpha=2.01,2.1,2.5$ and 2.99, as labelled). For low values of the cut-off, in the regime dominated by Coulomb cooling, the cooling time depends quite sensitively on $\alpha$. However, after a relatively short time, the cut-off of a cooling population moves to $q \sim 1$ and beyond, at which point the cooling shifts into the hadronically dominated regime, where it does not depend strongly on $\alpha$ any more.

rapidly thermalize the low momentum cosmic rays. The dotdashed line shows the total loss timescale, defined by

$\frac{1}{\tau_{\text {losses }}(q)}=\frac{1}{\tau_{\mathrm{C}}(q)}+\frac{1}{\tau_{\text {had }}(q)}$.

This timescale is monotonically rising with $q$. In Fig. 6, we show how this total cooling timescale depends on the assumed slope $\alpha$. For low values of the cut-off, in the regime dominated by Coulomb cooling, the cooling time depends quite sensitively on $\alpha$. However, after a relatively short time, the cut-off of a cooling population moves to $q \sim 1$ and beyond, at which point the cooling shifts into the hadronically dominated regime, where it does not depend strongly on $\alpha$ any more. If our prescribed $\alpha$ should not match the real slope of the CR spectrum well, there may hence be significant inaccuracies in the prompt cooling of the population, but the long-term errors should still be quite moderate.

In the absence of any injection, the cosmic ray population will always evolve towards a fixed cut-off $q_{\mathrm{fix}}$, driven by the tendency of Coulomb cooling to increase the cut-off, while catastrophic losses tend to lower it. A balance is reached at the solution of the equation

$1+\frac{\tau_{\mathrm{C}}\left(q_{\mathrm{fix}}\right)}{\tau_{\mathrm{had}}\left(q_{\mathrm{fix}}\right)}=\frac{T_{\mathrm{CR}}\left(\alpha, q_{\mathrm{fix}}\right)}{T_{\mathrm{p}}\left(q_{\mathrm{fix}}\right)}$,

which follows from Eqs. (15) and (16). The vertical dotted line in Fig. 5 marks this equilibrium point at $q_{\text {fix }}=1.685$ for $\alpha=2.5$. Once this fix-point is reached, only the spectral amplitude decays due to the cosmic ray cooling and dissipation processes. Finally, note that both the Coulomb cooling and the hadronic cooling time are inversely proportional to density. This also means that the fix-point $q_{\text {fix }}$ is density independent, but whether it can be reached in the available time at low density is a separate question.

It is also interesting to compare the cosmic ray loss timescale to the thermal cooling timescale of primordial gas. The latter is also inversely proportional to density, but has in addition a strong temperature dependence. In the bottom panel of Fig. 5, we show the thermal cooling timescale as a function of temperature, at the same fiducial density used in the top panel. For comparison, we show the asymptotic cosmic ray dissipation time scale (dashed line), which is reached if the cosmic ray population is dominated by the relativistic regime. In this case, cosmic rays decay much slower than the thermal gas pressure in the intermediate temperature regime. This could be interesting for cooling flows in halos or clusters. Even a moderate cosmic ray pressure contribution in the diffuse gas in a halo of temperature $T_{\text {vir }} \sim 10^{5}-10^{7} \mathrm{~K}$ should tend to survive longer than the thermal gas pressure, which could influence the cooling rate. We will examine this question explicitly in our numerical simulations of isolated halos.

\subsection{Equilibrium between source and sink terms}

The above considerations lead to an interesting question: What will the cosmic ray spectrum look like for a fluid element at a density $\rho$ high enough to allow star formation, such that is fed at a constant rate by supernova injection? We expect that after some time, a balance will be established between the supernova input on one hand and the cosmic ray losses due to Coulomb cooling and hadronic processes on the other hand. The energy content in the cosmic rays at this equilibrium point will then determine the $\mathrm{CR}$ pressure, and comparison of this pressure with the thermal pressure of the ISM will show whether "cosmic ray feedback" could be important in regulating star formation in galaxies.

To derive this equilibrium point, we first note that from the conditions $\Delta \tilde{\varepsilon}_{\mathrm{SN}}+\Delta \tilde{\varepsilon}_{\mathrm{C}}+\Delta \tilde{\varepsilon}_{\mathrm{had}}=0$ and $\Delta \tilde{n}_{\mathrm{SN}}+\Delta \tilde{n}_{\mathrm{C}}=0^{2}$ it directly follows that the mean energy per injected particle due to supernovae in equilibrium must be

$T_{\mathrm{CR}}\left(\alpha_{\mathrm{SN}}, q_{\mathrm{inj}}\right)=T_{\mathrm{p}}\left(q_{\mathrm{eq}}\right)\left[1+\frac{\tau_{\mathrm{C}}\left(q_{\mathrm{eq}}\right)}{\tau_{\mathrm{had}}\left(q_{\mathrm{eq}}\right)}\right]$.

This is a relation between the effective injection cut-off of the supernova feeding, and the equilibrium cut-off $q_{\text {eq }}$ of the CR spectrum. In equilibrium, we also know that the supernova input will just balance the cooling losses for the spectrum with equilibrium cut-off $q_{\text {eq }}$, yielding

$\tilde{\varepsilon}=\tau_{\text {losses }}\left(q_{\text {eq }}\right) f\left(q_{\mathrm{SN}}, q_{\text {inj }}\right)\left(\frac{\mathrm{d} \tilde{\varepsilon}}{\mathrm{d} t}\right)_{\mathrm{SN}}$.

On the other hand, our injection criterion of Eq. (18) tells us that $\tilde{\varepsilon}=\tau_{\text {losses }}\left(q_{\text {inj }}\right) f\left(q_{\mathrm{SN}}, q_{\text {inj }}\right)\left(\frac{\mathrm{d} \tilde{\varepsilon}}{\mathrm{d} t}\right)_{\mathrm{SN}}$, provided a solution for Eq. (18) actually exists when the system is in the dynamic equilibrium. Assuming this for the moment, it follows that the CR loss timescales at $q_{\text {inj }}$ and $q_{\text {eq }}$ are equal, which in turn implies $q_{\text {inj }}=q_{\text {eq }}$. In other words, the injection cut-off coincides with the cut-off of the equilibrium spectrum. The location of the equilibrium cut-off itself is given as solution of

$1+\frac{\tau_{\mathrm{C}}\left(q_{\mathrm{eq}}\right)}{\tau_{\mathrm{had}}\left(q_{\mathrm{eq}}\right)}=\frac{T_{\mathrm{CR}}\left(\alpha_{\mathrm{SN}}, q_{\mathrm{eq}}\right)}{T_{\mathrm{p}}\left(q_{\mathrm{eq}}\right)}$.

This is almost identical to Eq. (40) which describes the fix-point of the cut-off if there is no injection. The only difference is the occurrence of $\alpha_{\mathrm{SN}}$ instead of just $\alpha$ in the argument of the $T_{\mathrm{CR}}$ function. The result of this will be a slight shift of the equilibrium position once the assumed spectral indices for supernova injection and the general cosmic ray population differ, while for $\alpha_{\mathrm{SN}}=\alpha$, there will be no difference. At first sight it may seem

$\overline{2}$ Note that we always have $\Delta \tilde{n}_{\text {had }}=0$. 
surprising that the equilibrium position can be shifted away from the fix-point by an arbitrarily small supernova injection rate. However, recall that in the injection case we have described a truly invariant spectrum with a fixed amplitude (which may take very long time to be established), while in the case without injection, the amplitude keeps falling on the cooling timescale.

The above assumed that the injection condition (18) has a solution in equilibrium. This will be the case if $q_{\mathrm{eq}}$ determined by Eq. (43) is smaller or equal than $q_{\max }$ as given by Eq. (22). Otherwise the injection cut-off is given by $q_{\mathrm{inj}}=q_{\mathrm{max}}$, and the position of the equilibrium cut off is determined by Eq. (42). A detailed comparison of the steady-state CR spectrum with and without our approximate description is provided by Enßlin et al. (2007). There it is shown that dynamically important quantities like cosmic ray pressure and energy are calculated with $\sim 10 \%$ accuracy by our formalism.

Of primary importance for us is the equilibrium value of the cosmic ray energy content, because this will directly determine the CR pressure and hence the strength of potential feedback effects on star formation. The total cosmic ray energy injection rate by supernovae is related to the star formation rate by Eq. (31). Once the equilibrium cut-off $q_{\text {eq }}$ is known, the energy content in equilibrium will be given by Eq. (42), so that the pressure is fully specified. For example, in the case of $q_{\mathrm{eq}} \leq q_{\max }$, the final pressure is therefore given by

$P_{\mathrm{CR}}=\frac{(\alpha-1) \beta_{\alpha}\left(q_{\mathrm{eq}}\right) \tau_{\mathrm{loss}}\left(q_{\mathrm{eq}}\right) f_{\alpha_{\mathrm{SN}}}\left(q_{\mathrm{SN}}, q_{\mathrm{eq}}\right)}{3 \beta_{\alpha}\left(q_{\mathrm{eq}}\right)+6 q_{\mathrm{eq}}^{1-\alpha}\left(\sqrt{1+q_{\mathrm{eq}}^{2}}-1\right)} \zeta_{\mathrm{SN}} \epsilon_{\mathrm{SN}} \dot{\rho}_{\star}$.

In our simulations, we combine the cosmic ray formalism with the subresolution multiphase model for the regulation of star formation by Springel \& Hernquist (2003a). In the latter, the mean star formation rate is determined by the local density, and scales approximately as $\dot{\rho}_{\star} \propto \rho^{1.5}$ above a density threshold for the onset of star formation. A detailed discussion of the multiphase model together with the precise density dependence of the star formation rate is given in Springel \& Hernquist (2003a). These authors also derive an effective equation of state for the ISM, which governs the assumed two-phase structure of the ISM.

In Fig. 7, we show this effective pressure as a function of density, using the parameters for gas consumption timescale, initial mass function, and cloud evaporation efficiency discussed by Springel \& Hernquist (2003a), which result in a star formation threshold of $\rho_{\text {th }}=2.4 \times 10^{-25} \mathrm{~g} \mathrm{~cm}^{-3}$. We also plot the expected cosmic ray pressure in the same diagram, for two different injection efficiencies of $\zeta_{\mathrm{SN}}=0.1$ and $\zeta_{\mathrm{SN}}=0.3$. Quite interestingly, the cosmic ray pressure exceeds the thermal pressure at the threshold density for star formation, but due to the shallow dependence of the equilibrium pressure on density, with approximately $P_{\mathrm{CR}} \sim \rho^{0.5}$ (note that $\tau_{\text {losses }} \propto \rho^{-1}$ ), we find that the pressure of cosmic rays only plays a role for the low density part of the ISM model, while regions with very high specific star formation rates should be at most weakly affected.

For small efficiencies $\zeta_{\mathrm{SN}} \leq 0.01$, we essentially expect no significant influence of cosmic rays from supernova on the regulation of star formation whatsoever. Even for the fiducial choice of $\zeta_{\mathrm{SN}}=1$, the influence would vanish for densities $\rho>100 \rho_{\text {th }}$. Based on these findings, we expect that galaxies which form most of their stars at comparatively low densities should be potentially strongly affected by the cosmic ray feedback, while this influence should be weak or absent for vigorously star-forming galaxies with higher typical ISM densities. Our numerical results presented later will confirm this picture. The fact that CR-pressure seems to become dominant around

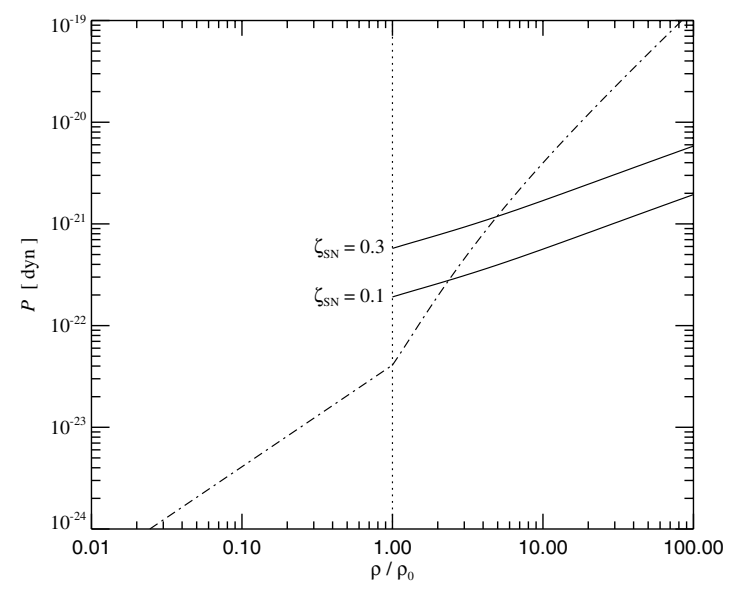

Fig. 7. Pressure of the cosmic ray population predicted for equilibrium between supernova injection on one hand, and Coulomb cooling and catastrophic losses on the other hand. The solid lines mark the pressure as a function of overdensity for two values of the injection efficiency $\zeta_{\mathrm{SN}}$. The assumed threshold density for star formation, $\rho_{0}=2.4 \times 10^{-25} \mathrm{~g} \mathrm{~cm}^{-3}$, is derived from the multiphase model of Springel \& Hernquist (2003a). The latter also predicts an effective equation of state for the star forming phase, shown as a dot-dashed line. The part below the threshold is an isothermal equation of state with temperature $10^{4} \mathrm{~K}$.

the star formation threshold may even suggest that cosmic rays could play an active role in defining this threshold.

\section{Cosmic ray diffusion}

Our treatment is based on the notion that the cosmic ray population is approximately locked into a gas fluid element by a locally present magnetic field. Even a weak ambient field makes the charged particle gyrate around the field lines, preventing them from freely travelling over macroscopic distances with their intrinsic velocity close to to the speed of light. The cosmic ray particles may move slowly along the field lines, but their perpendicular transport is strongly suppressed.

However, occasional scattering of particles on magnetic irregularities of MHD waves can displace the gyrocentres of particles, such that a particle effectively "changes its field line", allowing it to move perpendicular to the field. Since realistic magnetic field configurations are often highly tangled, or even chaotic, this can lead to sizable cross-field speeds of the particles. From a macroscopic point of view, the motion of the cosmic ray population can be described as a diffusion process, which is anisotropic with respect to the local magnetic field configuration. The theory of the respective diffusion coefficients is complicated, and uncertain for the case of turbulent magnetic field configurations (e.g. Rechester \& Rosenbluth 1978; Duffy et al. 1995; Bieber \& Matthaeus 1997; Giacalone \& Jokipii 1999; Narayan \& Medvedev 2001; Enßlin 2003).

In principle, one could try to simulate the magnetic field in $\mathrm{SPH}$ and then treat the diffusion in an anisotropic fashion. While recent advances in modelling MHD with SPH are promising (Dolag et al. 1999; Price \& Monaghan 2004), these techniques still face severe numerical challenges when applied to simulations with radiative cooling. We therefore defer such an approach to future work and model the diffusion isotropically. Also, since we have no direct information about the local magnetic field strength and field configuration, we will invoke a phenomenological approach to estimate the effective diffusion coefficient as 
a function of the local conditions of the gas. It would be easy however to refine the spatial dependence of the diffusion coefficient once a more detailed physical scenario becomes available.

Assuming isotropy, the diffusion in the $\mathrm{CR}$ distribution function $f(p, \boldsymbol{x}, t)$ can be written as

$$
\frac{\partial f}{\partial t}=\nabla(\kappa \nabla f)
$$

The diffusion coefficient $\kappa$ itself depends on the particle momentum (more energetic particles diffuse faster), and on the local magnetic field configuration. For definiteness we assume that the underlying MHD is turbulent with a Kolmogorov power spectrum, in which case the momentum dependence of $\kappa$ is given by

$\kappa(p)=\tilde{\kappa} p^{\frac{1}{3}}$,

where we assumed relativistic particle velocities with $v \simeq c$. Integrating the diffusion equation over particle momenta then yields

$$
\left.\frac{\partial n_{\mathrm{CR}}}{\partial t}\right|_{\mathrm{diff}}=\frac{\alpha-1}{\alpha-\frac{4}{3}} \nabla \tilde{\kappa} \nabla\left(q^{\frac{1}{3}} n_{\mathrm{CR}}\right),
$$

where $q$ is the spectral cut-off. Because more energetic particles diffuse faster, we expect that the diffusion speed for the cosmic ray energy density will be a bit higher than for the number density itself. To account for this effect, we first multiply the diffusion Eq. (45) with $T_{\mathrm{p}}(p)$, and then integrate over the particle momenta. This results in

$$
\left.\frac{\partial \varepsilon_{\mathrm{CR}}}{\partial t}\right|_{\mathrm{diff}}=\frac{\alpha-1}{\alpha-\frac{4}{3}} \nabla \tilde{\kappa} \nabla\left(q^{\frac{1}{3}} \frac{T_{\mathrm{CR}}\left(\alpha-\frac{1}{3}, q\right)}{T_{\mathrm{CR}}(\alpha, q)} \varepsilon_{\mathrm{CR}}\right),
$$

where $\varepsilon_{\mathrm{CR}}=\rho \tilde{\varepsilon}$ is the cosmic ray energy density per unit volume. The factor $T_{\mathrm{CR}}\left(\alpha-\frac{1}{3}, q\right) / T_{\mathrm{CR}}(\alpha, q)$ is larger than unity and encodes the fact that the diffusion in energy density proceeds faster than in particle number density. In Enßlin et al. (2007), we also give more general formulae for different power-law dependences of the diffusivity, and provide a more accurate treatment where the reduction of the diffusion rate at sub-relativistic energies is accounted for.

\subsection{Modelling the diffusivity}

Due to the lack of direct local information about the magnetic field strength in our present numerical models, we parameterize the dependence of the diffusion coefficient on local gas properties in terms of a fiducial power-law dependence on the local gas density and gas temperature. In particular, we make the ansatz

$\tilde{\kappa}=\kappa_{0}\left(\frac{\rho}{\rho_{0}}\right)^{n_{\rho}}\left(\frac{T}{T_{0}}\right)^{n_{T}}$,

for the diffusion constant, which is effectively a three parameter model for the diffusivity, specified by the overall strength $\kappa_{0}$ of the diffusion at a reference density and temperature, and by the power-law slopes $n_{\rho}$ and $n_{T}$ for the density and temperature dependence, respectively.

While clearly a schematic simplification, this parameterization is general enough to allow an analysis of a number of interesting cases, including models where the typical magnetic field strength has a simple density dependence, which can be a reasonable first order approximation for some systems, for example for the diffuse gas in cluster atmospheres (Dolag et al. 2004b).
For definiteness, we now construct such a very simple model, which will be the fiducial choice for the results on diffusion presented in this study. In Kolmogorov-like MHD turbulence, the dominant parallel diffusivity is expected to scale as (Enßlin 2003)

$\tilde{\kappa} \propto l_{B}^{2 / 3} B^{-1 / 3}$,

where $l_{B}$ gives a characteristic length scale for the magnetic field of strength $B$. We start out by assuming that the magnetic energy density is a fixed fraction of the thermal energy content, which corresponds to

$B \propto \rho^{1 / 2} T^{1 / 2}$.

An appropriate length scale for $l_{B}$ is more difficult to estimate, as it will sensitively depend on the level of local MHD turbulence, and on the build up of the magnetic field due to structure formation processes. For simplicity, we here assume that the length scale is related to the local Jeans scale, which may be appropriate for the conditions of a multiphase interstellar medium where local density fluctuations constantly form clouds of order a Jeans mass, which are then in part dispersed by supernova-driven turbulence. This then gives a scaling of the form

$l_{B} \propto \rho^{-1 / 2} T^{1 / 2}$.

Combining Eqs. (51) and (52), we obtain a model for the conductivity in the form $\tilde{\kappa} \propto T^{1 / 6} \rho^{-1 / 2}$, i.e. $n_{T}=1 / 6$ and $n_{\rho}=-1 / 2$. We fix the overall strength by alluding to measurements in our own Galaxy (Berezinskii et al. 1990; Schlickeiser 2002), which estimate a diffusivity along the magnetic field lines in the interstellar medium of approximately

$\kappa_{\mathrm{ISM}} \approx 3 \times 10^{27} \frac{\mathrm{cm}^{2}}{\mathrm{~s}} \approx 10 \frac{\mathrm{kpc}^{2}}{\mathrm{Gyr}}$.

Adopting our typical temperature and density values of the ISM as reference values, we end up with the following model for the diffusivity

$\tilde{\kappa}=10 \frac{\mathrm{kpc}^{2}}{\mathrm{Gyr}}\left(\frac{T}{10^{4} \mathrm{~K}}\right)^{1 / 6}\left(\frac{\rho}{10^{6} M_{\odot} \mathrm{kpc}^{-3}}\right)^{-1 / 2}$.

We will use this parameterization in the simulations with diffusion analysed in the this study. It is clear however that this model needs to be interpreted with a lot of caution, as the real diffusivity is highly uncertain, and may vary widely between different parts of the Universe. Improving the physical understanding of the strength of the diffusion will therefore remain an important goal for cosmic ray physics in the future.

\subsection{Discretizing the diffusion equation}

We still have to discuss how we numerically implement diffusion in our Lagrangian SPH code. We here follow a similar strategy as in Jubelgas et al. (2004), where a new treatment of thermal conduction in SPH was discussed and applied to simulations of clusters of galaxies. In essence, the very same techniques that can be used to solved the heat diffusion equation can also be applied to the cosmic ray diffusion needed here.

We first rewrite the diffusion equations into a Lagrangian form that is matched to the variables evolved in our simulation code. This results in

$\rho \frac{\mathrm{d} \tilde{\varepsilon}}{\mathrm{d} t}=\nabla \tilde{\kappa} \nabla D_{\varepsilon}$ 
and

$\rho \frac{\mathrm{d} \tilde{n}}{\mathrm{~d} t}=\nabla \tilde{\kappa} \nabla D_{n}$

where we have defined the abbreviations

$D_{\varepsilon}=\frac{\alpha-1}{\alpha-\frac{4}{3}} \rho q^{1 / 3} \frac{T_{\mathrm{CR}}\left(\alpha-\frac{1}{3}, q\right)}{T_{\mathrm{CR}}(\alpha, q)} \tilde{\varepsilon}$

and

$D_{n}=\frac{\alpha-1}{\alpha-\frac{4}{3}} \rho q^{1 / 3} \tilde{n}$

respectively. Our method for representing these equations in $\mathrm{SPH}$ is based on a discretisation scheme for the Laplace operator that avoids second order derivatives of the SPH kernel (Brookshaw 1985; Monaghan 1992), which makes the scheme robust against particle disorder and numerical noise. This gives us an evolution equation in the form of

$\frac{\mathrm{d} \tilde{\varepsilon}_{i}}{\mathrm{~d} t}=\sum_{j} \frac{m_{j}}{\rho_{i} \rho_{j}} \frac{2 \bar{\kappa}_{i j}\left(\bar{D}_{\varepsilon, j}-D_{\varepsilon, i}\right)}{\left|\boldsymbol{x}_{i j}\right|^{2}} \boldsymbol{x}_{i j} \nabla_{i} W_{i j}$,

and similarly for the cosmic ray number density. We here introduced a symmetrization of the diffusivities according to

$\bar{\kappa}_{i j}=2 \frac{\tilde{\kappa}_{i} \tilde{\kappa}_{j}}{\tilde{\kappa}_{i}+\tilde{\kappa}_{j}}$,

based on the suggestion by Cleary \& Monaghan (1999). Furthermore, we replaced one of the $D_{\varepsilon}$ terms in the pairwise diffusion term by the kernel interpolant

$\bar{D}_{\varepsilon, j}=\sum_{k} \frac{m_{k} D_{\varepsilon, k}}{\rho_{k}} W_{j k}$.

As Jubelgas et al. (2004) show, such a mixed formulation between intrinsic particle variables and SPH-smoothed interpolants substantially improves the numerical stability against small-scale particle noise. The smoothing process suppresses strong small-scale gradients, while long-range variations and their diffusive evolution remain unchanged. Since we use an explicit time integration scheme, this behaviour prevents stability problems due to the typical "overshooting" problem that otherwise may arise due to strong local gradients from local outliers.

Nevertheless, we still need to impose a comparatively strict timestep criterion to ensure proper integration of the diffusion. For the thermal conduction problem, we employed a simple criterion that limits the relative change of thermal energy of a particle within a single timestep. Although the diffusion studied here is in principle very similar to the conduction problem, an equivalent criterion is not a good choice, simply because unlike for thermal conduction, the relevant reservoir can often be empty. In fact, we typically start simulations from initial conditions where all the cosmic ray particle densities are identical to zero.

However, a closer look at the Green's function $G(x, t)=$ $(4 \pi \kappa t)^{-3 / 2} \exp \left[-x^{2} /(4 \kappa t)\right]$ of the diffusion process shows that differences between two points with a distance of $|\boldsymbol{x}|$ are diffused away with a characteristic timescale $x^{2} / \kappa$. Using the mean interparticle separation of SPH particles for the distance, this suggests the definition of a diffusion timescale in the form

$t_{\mathrm{diff}}=\frac{1}{\kappa}\left(\frac{m}{\rho}\right)^{2 / 3}$.
We use this to limit the maximum timestep for particles to be constrained by

$\Delta t<\varepsilon t_{\mathrm{diff}}=\varepsilon \frac{1}{\kappa}\left(\frac{m}{\rho}\right)^{2 / 3}$

where we used $\varepsilon=0.1$ for the simulations presented in this study. This has provided us with a numerically stable cosmic ray diffusion while at the same time timesteps are prevented from becoming impractically small.

As an additional refinement to the implementation of diffusion, we have implemented the method proposed by Jubelgas et al. (2004) to obtain a manifestly conservative scheme for cosmic ray energy and particle number, even when individual and adaptive timesteps are used. To this end, we rewrite Eq. (59) as

$m_{i} \frac{\mathrm{d} \tilde{\varepsilon}_{i}}{\mathrm{~d} t}=\sum_{j} \frac{\mathrm{d} E_{i j}}{\mathrm{~d} t}$

where we have defined a pairwise exchange term of cosmic ray energy in the form

$\frac{\mathrm{d} E_{i j}}{\mathrm{~d} t}=\frac{m_{i} m_{j}}{\rho_{i} \rho_{j}} \frac{2 \bar{\kappa}_{i j}\left(\bar{D}_{\varepsilon, j}-D_{\varepsilon, i}\right)}{\left|\boldsymbol{x}_{i j}\right|^{2}} \boldsymbol{x}_{i j} \nabla_{i} W_{i j}$

In each system step, we now determine the change of the cosmic ray energy of particle $i$ according to

$m_{i} \Delta \tilde{\varepsilon}_{i}=\frac{1}{2} \sum_{j k} \Delta t_{j}\left(\delta_{i j}-\delta_{i k}\right) \frac{\mathrm{d} E_{j k}}{\mathrm{~d} t}$.

The double sum on the right can be simply evaluated by the ordinary SPH sums over the active particles, provided that for each neighbour $j$ found for a particle $i$ one records a change of $\left(\Delta t_{i} / 2\right) \mathrm{d} E_{i j} / \mathrm{d} t$ for $i$, and a change of $-\left(\Delta t_{i} / 2\right) \mathrm{d} E_{i j} / \mathrm{d} t$ for the particle $j$. We then apply the accumulated changes of the cosmic ray energy (or particle number) to all particles at the end of the step, i.e. not only to the ones that are active on the current timestep. In this way, we arrive at a scheme that manifestly conserves total cosmic ray energy and number density for each diffusive step.

Finally, we note that we have implemented a further refinement in order to cope with technical problems associated with the situation in which isolated CR-pressurized particles are embedded in a background of particles with zero CR pressure. In the neighbourhood of such an isolated particle, the smoothed cosmic ray energy field $\bar{D}_{\varepsilon, j}$ will be non-zero for particles that have themselves no CR component (yet). This can then in turn lead to exchange terms $E_{i j}$ between particles which both have zero cosmic ray pressure, leading to unphysical negative values for the energy in one of them. We found that this can be avoided if we limit the value of the interpolant $\bar{D}_{\varepsilon, i}$ to be no more than a factor $\chi \simeq 2.0$ larger than the value $D_{\varepsilon, i}$ for the particle itself. With this change, we recovered numerical stability of the diffusion in this situation.

\section{Numerical details and tests}

The numerical framework for cosmic ray physics presented above allows for very complex dynamics that interacts in nonlinear and rather non-trivial manner with different aspects of ordinary hydrodynamics, and in particular with the physics of radiative cooling and star formation included in GADGET-2 to describe galaxy formation. Together with the nearly complete absence of analytic solutions, this makes a direct validation of 
the numerical implementation of cosmic ray physics in the simulation code particularly hard.

However, there are a few areas where the careful checks of individual subroutines of the code that we carried out can be augmented with tests of problems where analytical solutions are known. One such area are hydrodynamical shock waves that involve a cosmic ray pressure component. This allows us to test one of the most interesting dynamical aspects of cosmic ray physics which is the introduction of a variable adiabatic index $\gamma$. Note that the pressure of a "hybrid gas" with a thermal and a cosmic ray energy density can no longer be described with the simple parameterizations used for a polytropic gas. In particular, both the relative contribution of the cosmic ray pressure and the adiabatic index of the $\mathrm{CR}$ pressure component itself will change during an adiabatic compression, and in addition, new cosmic rays can be accelerated at a shock front. While more complex than for an ideal gas, the Riemann problem for a shocks in such a composite gas can be solved analytically (Pfrommer et al. 2006), and we will use this as a test for our numerical treatment.

Another aspect which can be tested with simple toy set-ups is the diffusion of cosmic rays. To this end we will consider a geometrically simple initial cosmic ray distribution, together with the gas being forced to be at rest. This allows us to test the correct diffusion speed, and the conservative properties of the diffusion process.

\subsection{Implementation issues}

When the mean CR energy is updated, we need to numerically invert the specific energy $T_{\mathrm{CR}}$ of Eq. (10) for $q$, which we do efficiently by means of pre-computed look-up tables, avoiding the need to frequently evaluate costly incomplete beta functions. Once the spectral cutoff and injected CR-to-baryon fraction is found, the new spectral normalization $\tilde{C}$ can be computed from Eq. (9). As a final step, we can then update the effective hydrodynamic pressure due to cosmic rays of the particle in question.

For treating Coulomb losses in an accurate and robust way, we use an implicit scheme because of the very sensitive dependence of the cooling rate on the spectral cut-off $q$. In fact, in order to ensure that this cooling process leaves the normalization of the spectrum constant and only increases the cut-off $q$, we solve the following implicit equation

$\tilde{\varepsilon}\left(q^{\prime}, \tilde{C}\right)=\tilde{\varepsilon}(q, \tilde{C})-\frac{\tilde{\varepsilon}\left(q^{\prime}, \tilde{C}\right)}{\tau_{\mathrm{C}}\left(q^{\prime}\right)} \Delta t$

for a new spectral cut-off $q^{\prime}$ when the cooling lasts for a time interval $\Delta t$. This scheme is robust also in cases where $\Delta t$ exceeds $\tau_{\mathrm{C}}(q)$. Unlike the Coulomb losses, the timescale of hadronic losses is comparatively long and varies little with the spectral cut-off, as seen in Fig. 5. It therefore is easier to integrate accurately and does not require an implicit solver.

\subsection{Shocks in cosmic ray pressurized gas}

We performed a number of three-dimensional test simulations that follow a shock wave in a rectangular slab of gas, which is extended along one spatial dimension. Periodic boundary conditions in the directions perpendicular to this axis are used to make sure that no boundary-effects occur. This allows us to simulate a planar shock in 3D which can then be compared to a corresponding one-dimensional analytic solution. The initial conditions of our shock-tube tests were set-up with relaxed "glass" structures of particles initially at rest, and by giving the two halves of the slab different temperatures and cosmic ray pressures. The particle mass was constant and chosen such that the mean particle spacing was 1 length unit in the high-density regime. While a reduction of the particle mass in the low-density region would have resulted in an increased spatial resolution there, our constant particle mass set-up is more appropriate for the conditions encountered in cosmological simulations. We used 64 smoothing neighbours in the SPH calculations.

In the left panel of Fig. 8, we show the state of a shock-tube test after a time of $t=0.3$, for an initial density contrast of 5 , a total pressure ratio of 35.674 , a homogeneous mixture of $1 / 3$ cosmic ray pressure and $2 / 3$ thermal pressure contribution on the left-hand side, and pressure equilibrium between CRs and thermal gas on the right-hand side. A shock with Mach number $\mathcal{M}=3$ travelling to the right, a rarefaction wave to the left, and a contact discontinuity in between develop for these initial conditions. In this test, no new CRs were accelerated at the shock front. The analytically predicted shock front position and density distribution are matched nicely by the simulation. Due to the smooth nature of SPH simulations, the density jump at the shock at $x \simeq 430$ is not a sharp discontinuity, but stretches over a small number of mean interparticle spacings. The contact discontinuity at $x \simeq 375$ is reproduced well, with only a small "blip" seen in both the density and the pressure profile, which is characteristic for SPH in shock tube tests. Note that cosmic ray pressure dominates over thermal pressure on the left side of the contact discontinuity due to adiabatic rarefaction of the initially CR-dominated state on the left-hand side, while this is reversed on the other side because CRs are adiabatically compressed at the shock while the thermal gas experiences entropy injection. The rarefaction wave traveling to the left shows the expected behaviour over most of its extent, only in the leftmost parts at $x \simeq 100$ some small differences between the analytic and numerical solution are seen. However, the overall agreement is very reassuring, despite the fact that here a shock in a composite gas was simulated. The right panel of Fig. 8 shows another shock-tube test were the gas was initially made purely thermal, and the pressure ratio was increased to obtain a stronger shock wave of Mach number $\mathcal{M}=30$. In this simulation we have accounted for the acceleration of CRs at the shock, visible as a significant CR population in the post-shock region. Again, the match between the analytic solution and the simulation result is very satisfactory. Note that the density jump at the shock is larger than 4 (the maximum value for a purely thermal gas) due to the significant post-shock pressure support by freshly injected CRs that have a soft equation of state, which makes the composite gas more compressible compared to a plain thermal gas.

This shows that the simulation code is able to correctly follow rapid compressions and rarefactions in a gas with substantial cosmic ray pressure support, including shocks that feed their dissipated energy both into the thermal component and into the cosmic ray population. We also note that our shock detection technique (Pfrommer et al. 2006) is able to correctly identify the shock location on-the-fly during the simulation, and returns the right Mach number in the peak of the shock profile, where most of the energy is dissipated. We can use this to accurately describe the Mach-number dependent shock-injection efficiency of cosmic rays in shocks.

\subsection{Cosmic ray diffusion}

In order to test the diffusion part of the code, we use a system of gas particles that are at rest, which avoids the complications that would otherwise occur due to the motions of particles. We 

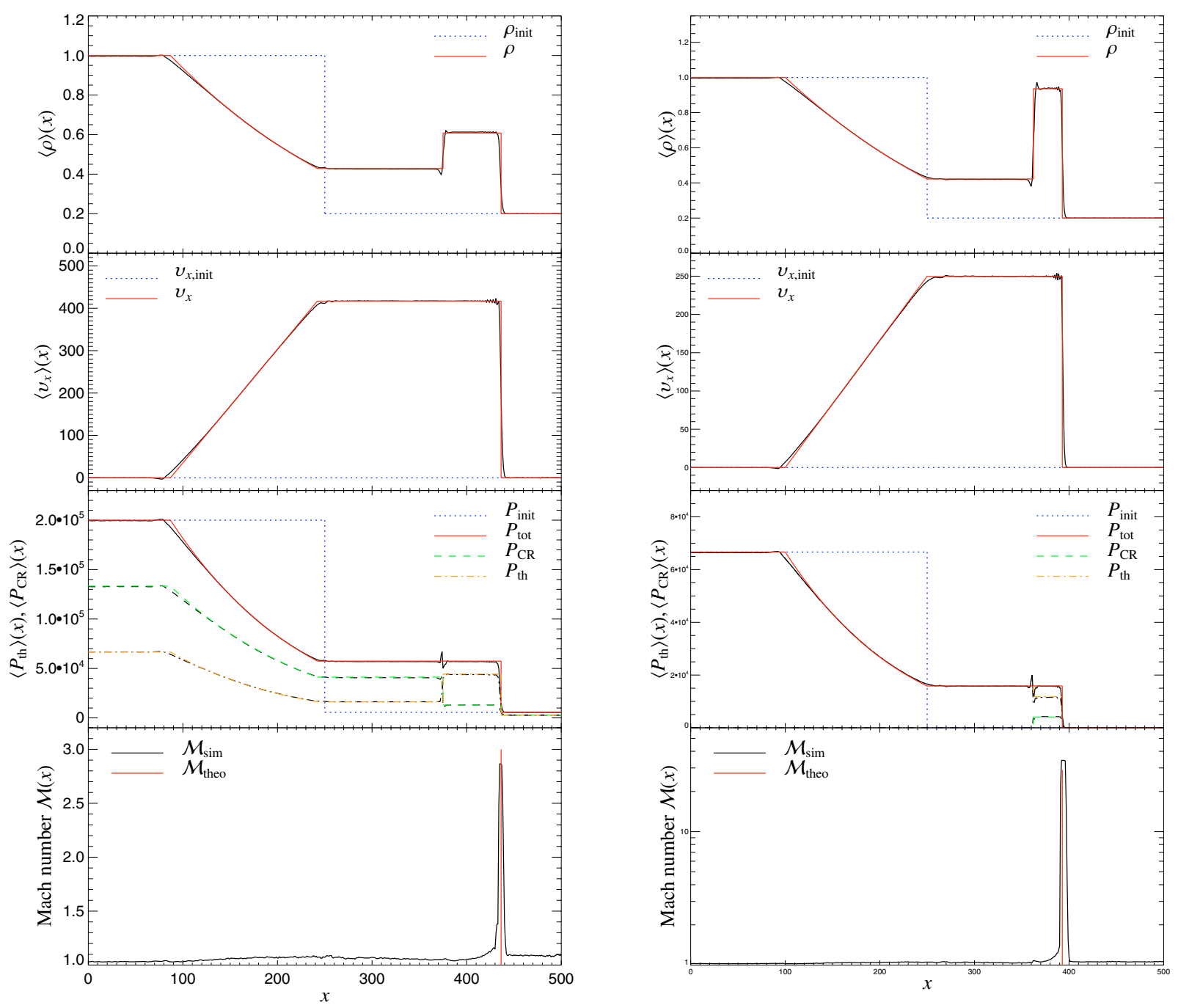

Fig. 8. Shock-tube tests for a gas with thermal and cosmic ray pressure components. The simulations are carried out in a three-dimensional periodic box which is longer in the $x$-direction than in the other two dimensions. The numerical result of the averaged hydrodynamical quantities of all SPH particles within bins with a spacing equal to the interparticle separation of the denser medium is shown in black and compared with the analytic result in colour. The typical rms-scatter in each bin among the particles is $\sim 10 \%$, reflecting the typical level of SPH noise for irregular particle distributions. The particle mass was constant and chosen such that the mean particle spacing was 1 length unit in the high-density regime (meaning that there were initially 250 particles along the $x$-axis in the left half and 146 in the right half of the tube). In the test shown on the left, the relative CR pressure is $X_{\mathrm{CR}}=P_{\mathrm{CR}} / P_{\mathrm{th}}=2$ in the left half-space $(x<250)$, while we assume pressure equilibrium between CRs and thermal gas for $x>250$. The evolution then produces a Mach number $\mathcal{M}=3$ shock wave. In this test, CR acceleration was disregarded at the shock front, so the cosmic ray component is merely adiabatically compressed by the shock. In the test shown on the right, the shock-tube was initially filled with purely thermal gas $(\gamma=5 / 3)$, and with a higher pressure ratio of 4625 at the initial interface. A shock with Mach number $\mathcal{M}=30$ develops and propagates to the right, and here we include cosmic ray acceleration at the shock front. We see that our numerical method correctly captures the production of cosmic rays by the shock, as the good agreement with the analytic solution of the Riemann problem demonstrates. To derive the latter (see Pfrommer et al. 2007, in preparation), we assumed a sufficiently strong shock that injects a hard population of CRs for which their ultra-relativistic equation of state holds.

achieve this by setting all particle accelerations to zero, implying that the densities remain constant over time. All variations in the distribution of cosmic rays are then entirely due to diffusive transport (we also switched off the Coloumb and catastrophic losses for this test). For this idealized situation, analytic solutions for the diffusion problem can be derived which can then be readily compared with numerical results.

For definiteness, we set up a periodic slab of matter with density $10^{10} M_{\odot} \mathrm{kpc}^{-3}$, spanning a basic volume of $10 \times 10 \times$ $100 \mathrm{kpc}^{3}$. The periodicity across the short dimensions ensures the absence of boundary effects, such that we can compare the numerical results to effectively one-dimensional analytic solutions. The cosmic ray distribution was initialized such that the energy density due to relativistic particles has a sharp step, being equal to $\tilde{\epsilon}_{1}$ for $x<0$, and equal to $\tilde{\epsilon}_{2}=\tilde{\epsilon}_{1} / 4$ for $x \geq 0$. The spectral cutoff at both sides of the step was set equal to $q=0.3$ in the test discussed here, but we note that identical results are also obtained for different choices. Again, we used an irregular glasslike configuration as initial particle distribution in order to realistically model the noise properties in density fields encountered in cosmological applications, and we employed 64 neighbours in the SPH calculations. Note that small-scale numerical noise can 

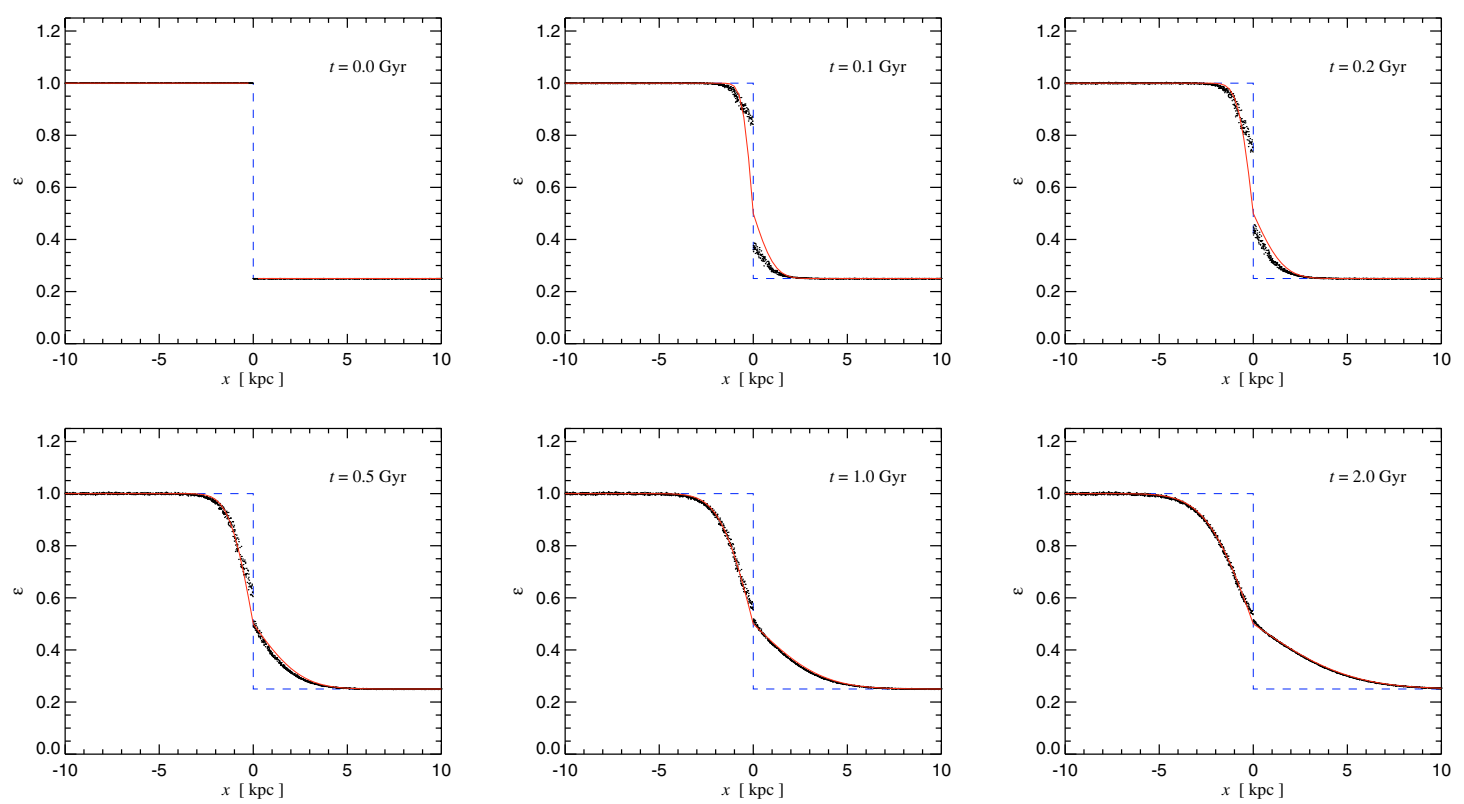

Fig. 9. Time evolution of a step function in cosmic ray energy density due to diffusion, for a gas that is at rest. The times shown in the different panels are (from top left to bottom right): $t=0,0.1,0.2,0.5,1.0$ and $2.0 \mathrm{Gyr}$. Black dots give particle values at the corresponding time, while the red line shows the analytical solution. The initial distribution is indicated by a thin dashed line. The diffusivity $\tilde{\kappa}$ is constant at $1 \mathrm{kpc}^{2} \mathrm{Gyr}^{-1} \mathrm{on}_{\mathrm{s}}$ left hand side, and four times higher on the right hand side. A "glass" like particle distribution was used with a mean particle separation of $1 \mathrm{kpc}$. A constant spectral cut-off and slope was assumed throughout the volume. The maximum deviation of SPH particles from the analytic results drops from $L_{\infty}=0.33$ at $t=0.1 \mathrm{Gyr}$ to $L_{\infty}=0.039$ at $t=2.0 \mathrm{Gyr}$, while the mean relative error per particle in the range $-10 \mathrm{kpc} \leq x \leq 10 \mathrm{kpc}$ is always below $L_{1} \leq 0.01$.

be problematic for our treatment of diffusion (e.g. Jubelgas et al. 2004), so this is an important aspect for testing the robustness of the implemented scheme.

In real physical applications, the diffusion implementation will have to deal with a spatially varying diffusion coefficient. In particular, there will be steep gradients in the diffusivity at phase transition between the cold, dense gas and the hot, yet thin ambient intergalactic and intra-cluster medium. It is therefore advisable to verify that the implemented numerical scheme for the diffusion is well behaved at sharp jumps of the diffusivity. We incorporate this aspect into our test scenario by introducing a step in the diffusivity at the initial interface, with $\kappa_{1}=1.0 \mathrm{kpc}^{2} \mathrm{Gyr}^{-1}$ in the left half of the slab, and a four times higher value of $\kappa_{2}=4.0 \mathrm{kpc}^{2} \mathrm{Gyr}^{-1}$ for the right half of the slab. In order to be able to compute an analytic solution, we will assume here that this diffusivity applies both to the CR energy density and CR number density.

In Fig. 9, we present the time evolution of this diffusion problem obtained with our numerical implementation. The simulation was run over a time span of $2 \mathrm{Gyr}$, and for a number of times in between, we compare the spatial distribution of the cosmic ray energy density obtained numerically with the analytical solution for the problem, which is given by

$$
\tilde{\epsilon}(x, t)=\left\{\begin{array}{l}
\tilde{\epsilon}_{1}+\frac{\tilde{\epsilon}_{2}-\tilde{\epsilon}_{1}}{1+\sqrt{\tilde{\kappa}_{1} / \tilde{\kappa}_{2}}}\left[\operatorname{erf}\left(\frac{x}{\sqrt{4 \tilde{\kappa}_{1} t}}\right)+1\right] \text { for } x<0 \\
\tilde{\epsilon}_{2}+\frac{\tilde{\epsilon}_{2}-\tilde{\epsilon}_{1}}{1+\sqrt{\tilde{\kappa}_{2} / \tilde{\kappa}_{1}}}\left[\operatorname{erf}\left(\frac{x}{\sqrt{4 \tilde{\kappa}_{2} t}}\right)-1\right] \text { for } x \geq 0 .
\end{array}\right.
$$

While some noticeable differences occur in the very early evolution of the initial discontinuity, the match of the numerical result and the analytic solution becomes quite good at later times. In fact, after $t=1 \mathrm{Gyr}$, we no longer see any significant deviation between the numerical solution and the analytical one. The fact that large differences occur in the very early phases of the evolution is not unexpected, given that SPH cannot easily represent sharp discontinuities. As a result of the smoothing inherent in SPH and of our diffusion formulation, sharp gradients on very small-scales are only washed out with some delay, but errors caused by this do not propagate to larger scales, such that the diffusion speed of large-scale gradients is quite accurate nevertheless. We note that we have verified the good accuracy of the diffusion results for a wide range of matter densities and diffusivities, including also cases with stronger spatial variations in diffusivity. We are hence confident that our numerical implementation should produce accurate and robust results in full cosmological simulations, where the diffusivity can show non-trivial spatial dependences.

\section{Simulations of isolated galaxies and halos}

We now turn to a discussion of the effects of our cosmic ray model on the galaxy formation process. Due to the complexity of the involved physics, which couples radiative cooling, star formation, supernova feedback, cosmic ray physics, self-gravity, and ordinary hydrodynamics, it is clear however that our analysis cannot be fully exhaustive in this methodology paper. Instead, our strategy is to provide a first exploration of the most important effects using a set of simulations with idealized initial conditions, and a restricted set of full cosmological simulations. This can then guide further in-depth studies of the individual effects.

One of the possible effects of cosmic ray physics is that the injection of CRs due to supernovae may alter the regulation of star formation by feedback, which may directly translate into observable differences in forming galaxies. Since CR-pressurized gas has a different equation of state than ordinary thermal gas, it may rise buoyantly from star-forming regions, which could perhaps help to produce outflows from galactic halos. Also, because energy stored in cosmic rays will be subject to different 
dissipative losses than thermal gas, we expect that the radiative cooling of galaxies could be altered. Of special importance is also whether the strength of any of these effects shows a dependence on halo mass, because a change of the efficiency of galaxy formation as a function of halo mass is expected to modify the shape of the resulting galaxy luminosity function.

Another intriguing possibility is that the total baryonic fraction ending up in galactic halos could be modified by the additional pressure component provided by the relativistic particle population. In particular, the softer equation of state of the cosmic-ray gas component (its adiabatic index varies in the range $4 / 3<\gamma_{\mathrm{CR}}<5 / 3$ ) could increase the concentration of baryonic matter in dark matter potential wells, because the pressure increases less strongly when the composite CR/thermal gas is compressed. On the other hand, a partial cosmic ray pressure support might reduce the overall cooling efficiency of gas in halos, causing a reduction of the condensated phase of cold gas in the centres.

To examine the non-linear interplay of all these effects, we will study them in a number of different scenarios. We first use isolated galaxy models which allow a precise control over the initial conditions and an easy analysis and interpretation of the results. Next, we use non-radiative cosmological simulations to investigate the efficiency of $\mathrm{CR}$ production at structure formation shock waves. We then use high-resolution cosmological simulations that include radiative cooling and star formation to study the formation of dwarf galaxies, with the aim to see whether our identified mass trends are also present in the full cosmological setting. We also use these simulations to investigate whether CRs influence the absorption properties of the intergalactic medium at high redshift. Finally, we use high-resolution "zoomed" simulations of the formation of clusters of galaxies to study how CR injection by accretion shocks and supernovae modifies the thermodynamic properties of the gas within halos.

\subsection{Formation of disk galaxies in isolation}

As a simple model for the effects of cosmic ray feedback on disk galaxy formation, we consider the time evolution of the gas atmospheres inside isolated dark matter halos. The initial conditions consist of a dark matter potential with a structure motivated from cosmological simulations, combined with a hydrostatic gas distribution initially in equilibrium within the halo. The halo carries a small amount of angular momentum, described by a spin parameter $\lambda=0.05$, which is close to the median found in cosmological simulations. We then consider the evolution of this system under radiative cooling, star formation and cosmic ray production by supernovae. We expect that the gas in the centre looses its pressure support by cooling, and then collapses into a rotationally supported disk that forms inside-out (Fall \& Efstathiou 1980).

It is clear that this is a highly idealized model for disk galaxy formation, which glosses over the fact that in a more realistic cosmological setting galaxies originate in a hierarchical process from the gravitational amplification of density fluctuation in the primordial mass distribution, gradually growing by accretion and merging with other halos into larger objects. However, the simplified approach we adopt here should still be able to capture some of the basic processes affecting this hierarchy, and it does so in a particular clean way that should allow us to identify trends with galaxy mass due to cosmic rays.

We model the dark matter and baryonic content of our isolated halos as NFW density profiles (Navarro et al. 1996), which we slightly soften at the centre to introduce a core into the gas density, with a maximum density value lying below the threshold for star formation, allowing for a "quiet" start of the simulations. The velocity dispersion of the dark matter and the temperature of the gas were chosen such that the halos are in equilibrium initially, i.e. when evolved without radiative cooling, the model halos are perfectly stable for times of order the Hubble time. We also impart angular momentum onto the halo with a distribution inside the halo consistent with results obtained from full cosmological simulations (Bullock et al. 2001).

We simulated a series of host halos with masses varying systematically between $10^{9} h^{-1} M_{\odot}$ and $10^{12} h^{-1} M_{\odot}$. In all cases, we adopted a baryon fraction of $\Omega_{\mathrm{b}} / \Omega_{\mathrm{m}}=0.133$, and a matter density of $\Omega_{\mathrm{m}}=0.3$. We typically represented the gas with $10^{5}$ particles and the dark matter with twice as many. In some of our simulations, we also replaced the live dark halo with an equivalent static dark matter potential to speed up the calculations. In this case, the contraction of the dark matter due to baryonic infall is not accounted for, but this has a negligible influence on our results. We have kept the concentration of the NFW halos fixed at a value of $c=12$ along the mass sequence, such that the initial conditions are scaled versions of each other which would evolve in a self-similar way if only gravity and ideal hydrodynamics were considered. However, this scale-invariance is broken by the physics of cooling, star formation and cosmic rays.

When one of these halos is evolved forward in time, radiative cooling leads to a pressure loss of the gas in the centre, which then collapses and settles into a rotationally supported cold disk. In the disk, the gas is compressed by self-gravity to such high densities that star formation ensues. Unfortunately, the physics of star formation is not understood in detail yet, and we also lack the huge dynamic range that would be necessary do directly follow the formation and fragmentation of individual star-forming molecular clouds in simulations of whole galaxies. In this study, we therefore invoke a sub-resolution treatment for star formation, in the form described by Springel \& Hernquist (2003a). The model assumes that the dense interstellar medium can be approximately described as a two-phase medium where cold clouds form by thermal instability out of a diffuse gaseous phase. The clouds are the sites of star formation, while the supernovae that accompany the star formation heat the diffuse medium, and, in particular, evaporate some of the cold clouds. In this way, a selfregulation cycle for star formation is established.

When our new cosmic ray model is included in our simulation code, a fraction of the deposited supernova energy is invested into the acceleration of relativistic protons, and hence is lost to the ordinary feedback cycle. While this energy no longer directly influences the star formation rate, it has an indirect effect on the star-forming gas by providing a pressure component that is not subject to the usual radiative cooling. If this pressure component prevails sufficiently long, it can cause the gas to expand and to lower its density, thereby reducing the rate of star formation.

In Fig. 10, we show the time evolution of the star formation rate for four different halos masses, ranging from $10^{9} h^{-1} M_{\odot}$ to $10^{12} h^{-1} M_{\odot}$. For each halo mass, we compare three different cases, a reference simulation where the ordinary model of Springel \& Hernquist (2003a) without cosmic rays was used, and two simulations where cosmic ray production by supernovae was included (without allowing for diffusion), differing only in the assumed efficiency of $\zeta_{\mathrm{SN}}=0.1$ and $\zeta_{\mathrm{SN}}=0.3$ for this process, respectively. The CR population was represented with a constant slope of $\alpha=2.5$. Interestingly, the simulations with cosmic rays show a substantial reduction of the star formation rate in the two small mass systems, but already for the $10^{11} h^{-1} M_{\odot}$ halo 

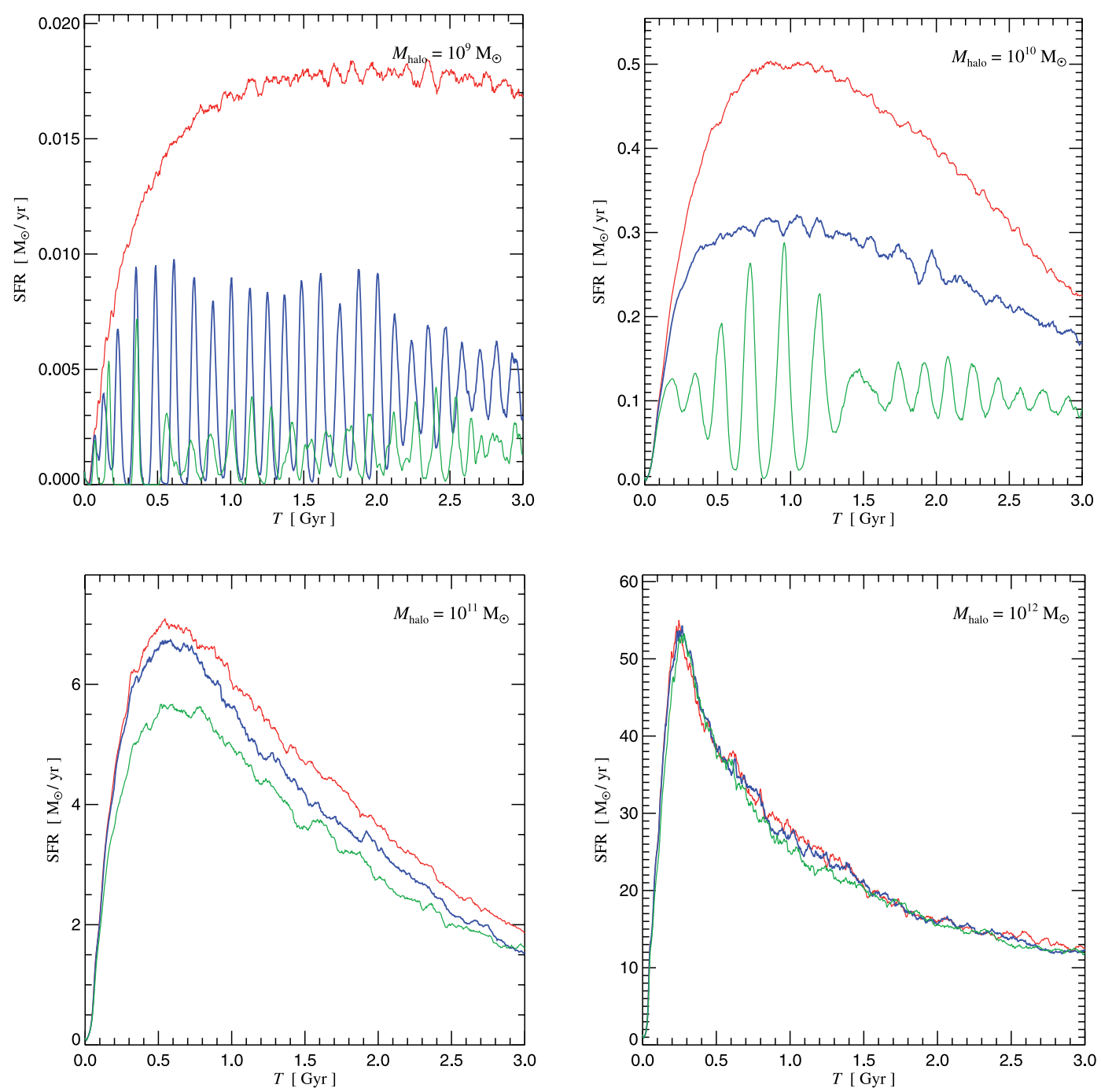

Fig. 10. Time evolution of the star formation rate in isolated halos of different mass which are initially in virial equilibrium. In each panel, we compare the star formation rate in simulations without cosmic ray physics (solid red line) to two runs with different injection efficiency of cosmic rays by supernovae, $\zeta_{\mathrm{SN}}=0.1$ (blue lines) and $\zeta_{\mathrm{SN}}=0.3$ (green lines), respectively. From top left to bottom right, results for halos of virial mass $10^{9} h^{-1} M_{\odot}$ to $10^{12} h^{-1} M_{\odot}$ are shown, as indicated in the panels. Efficient production of cosmic rays can significantly reduce the star formation rate in very small galaxies, but it has no effect in massive systems.

the effects becomes comparatively small, while for the massive halo of mass $10^{12} h^{-1} M_{\odot}$, no significant differences can be detected. Clearly, the ability of cosmic ray feedback to counteract star formation shows a rather strong mass dependence, with small systems being affected most. For higher efficiencies $\zeta_{\mathrm{SN}}$ of CR-production by supernovae, the reduction of the star formation rate becomes larger, as expected.

Figure 11 provides an explanation for this result, and also elucidates the origin of the oscillatory behaviour of the SFR in the CR-suppressed cases. In the figure, we show phase-space diagrams of the gas particles of the $10^{9} h^{-1} M_{\odot}$ and $10^{12} h^{-1} M_{\odot}$ halos, respectively, in a plane of effective temperature versus density. We plot the thermal pressure and the cosmic ray pressure separately. In order to cleanly show whether a dynamical effect of cosmic rays can be expected, we here use a fiducial simulation where the cosmic ray pressure is ignored in the equations of motion, but is otherwise computed with the full dynamical model. As Fig. 11 demonstrates, the bulk of the starforming gas in the massive halo lies at much higher density and higher effective pressure than in the low mass halo. Because the cosmic ray pressure exceeds the effective thermal pressure of the multi-phase ISM only for moderate overdensities relative to the star formation threshold, most of the gas in the $10^{12} \mathrm{~h}^{-1} M_{\odot}$ halo is simply too dense to be affected by the cosmic ray pressure. We note that the relative sizes of the two pressure components are consistent with the analytic expectations shown in Fig. 7. In fact, these expectations are replicated as dashed lines in Fig. 11 and are traced well by the bulk of the particles. Because the shallower potential wells in low-mass halos cannot compress the gas against the effective pressure of the ISM to comparably high overdensities as in high-mass halos, it is therefore not surprising that the cosmic ray pressure becomes dynamically important only in small systems.

Figure 11 also makes it clear that in the regime where cosmic ray pressure may dominate we cannot expect a dynamically stable quasi-equilibrium with a quiescent evolution of the star formation rate. This is simply due to the decline of the effective cosmic ray "temperature" $P_{\mathrm{CR}} / \rho$ with increasing density of the ISM, a situation which cannot result in a stable equilibrium configuration where self-gravity is balanced by the cosmic 

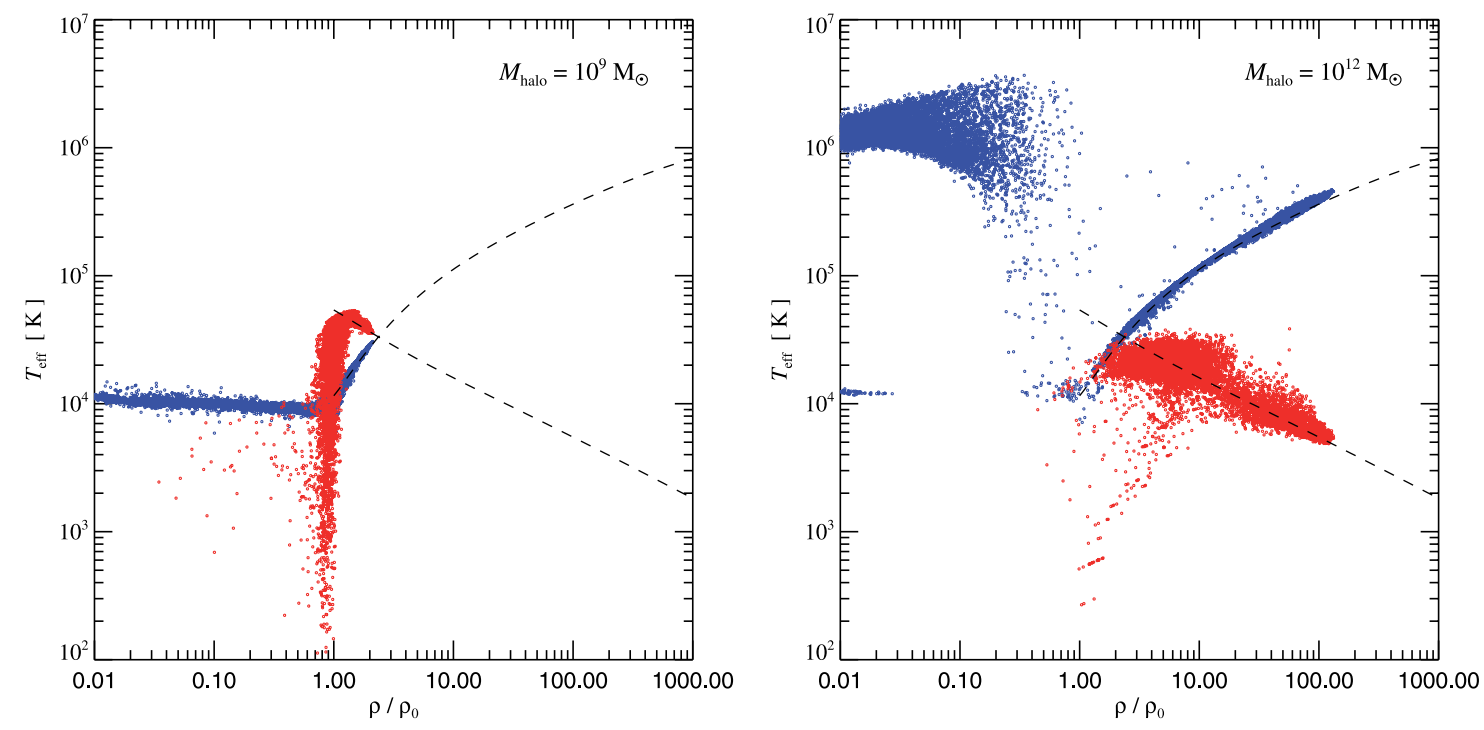

Fig. 11. Phase-space diagram of the star-forming phase in two simulations with halos of different mass. In these fiducial simulations, we included cosmic ray physics but ignored the cosmic ray pressure in the equations of motion, i.e. there is no dynamical feedback by cosmic rays. However, a comparison of the cosmic ray pressure and the thermal pressure allows us to clearly identify regions where the cosmic rays should have had an effect. For graphical clarity, we plot the pressures in terms of a corresponding effective temperature, $T_{\text {eff }}=(\mu / k) P / \rho$. Above the star formation threshold, the small galaxy of mass $10^{9} h^{-1} M_{\odot}$ shown in the left panel has a lot of gas in the low-density arm of the effective equation of state, shown by the curved dashed line. On the other hand, the massive $10^{12} h^{-1} M_{\odot}$ galaxy shown on the right has characteristically higher densities in the ISM. As a result, the cosmic ray pressure is insufficient to affect this galaxy significantly. Note that the falling dashed line marks the expected location where cosmic ray loss processes balance the production of cosmic rays by supernovae. We show the systems at time $t=2.0 \mathrm{Gyr}$ after the start of the evolution.

ray pressure. Instead, the system should be intrinsically unstable in this regime. When some gas becomes dense enough to start star formation, it will first have no cosmic ray pressure support but it will be stabilized against collapse by the thermal pressure of the ISM that is quickly established by supernova feedback. After some time, the ongoing star formation builds up a cosmic ray pressure component, which eventually starts to dominate, at which point the gas is driven to lower density. As a result, the star formation rate declines strongly. After some time, the CR pressure is dissipated such that the gas can collapse again. Star formation will then start again and the "cycle" can repeat. This scenario schematically describes the origin of the oscillations in the star formation rate seen in the results for the $10^{9} h^{-1} M_{\odot}$ and $10^{10} h^{-1} M_{\odot}$ halos when cosmic rays are included.

Another view of the halo mass dependence of the effects of cosmic ray feedback on star formation is given by Fig. 12. Here we show the integrated stellar mass formed up to time $t=3 \mathrm{Gyr}$, normalized by the total baryonic mass. Again, we compare two different injection efficiencies $\left(\zeta_{\mathrm{SN}}=0.1\right.$ and $\left.\zeta_{\mathrm{SN}}=0.3\right)$ with a reference case where no cosmic ray physics is included. In general, star formation is found to be most efficient on intermediate mass scales of $\sim 10^{11} M_{\odot}$ in these simulations. However, the simulations with cosmic ray production show a clear reduction of their integrated star formation rate for halos with mass below a few times $10^{11} h^{-1} M_{\odot}$, an effect that becomes progressively stronger towards lower mass scales. For the $10^{9} h^{-1} M_{\odot}$ halo, the suppression reaches more than an order of magnitude for $\zeta_{\mathrm{SN}}=0.3$.

In Fig. 13, we take a closer look at the spatial distribution of the cosmic ray pressure in the different cases, and the profiles of the stellar disks that form. To this end, we show the projected gas density distribution in an edge-on projection at time $t=2.0 \mathrm{Gyr}$, comparing the case without cosmic rays (left column) to the case with cosmic rays (middle column), for a range of halo masses from $10^{9} h^{-1} M_{\odot}$ to $10^{12} h^{-1} M_{\odot}$. For the simulation with

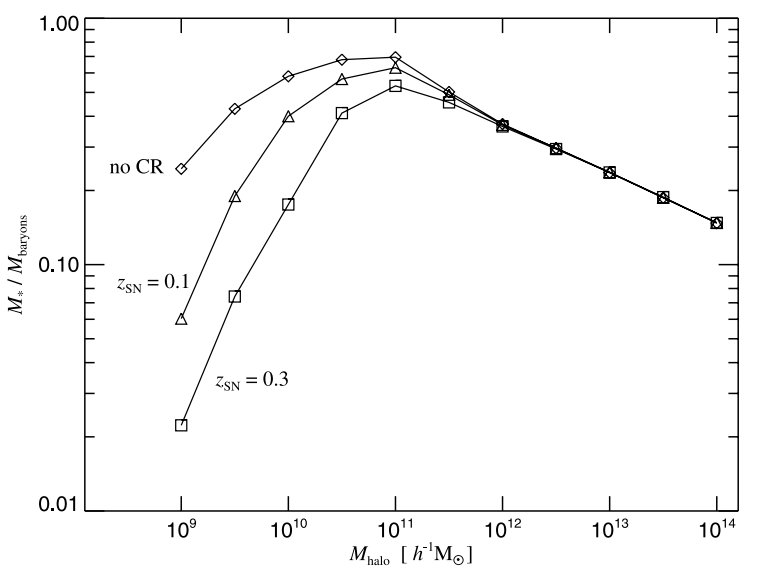

Fig. 12. Efficiency of star formation as a function of halo mass in our isolated disk formation simulations. We show the ratio of the stellar mass formed to the total baryonic mass in each halo, at time $t=3.0 \mathrm{Gyr}$ after the start of the simulations, and for two different efficiencies of cosmic ray production by supernovae. Comparison with the case without cosmic ray physics shows that star formation is strongly suppressed in small halos, by up to a factor $\sim 10-20$, but large systems are essentially unaffected.

cosmic rays, we overlay contours for the relative contribution of the projected cosmic ray energy to the total projected energy density. This illustrates, in particular, the spatial extent the cosmic ray pressure reaches relative to the star-forming region. Finally, the panels on the right compare surface density profiles of the stellar mass that has formed up to this time.

Consistent with our earlier results, the stellar density profiles of the low mass halos show a significant suppression when cosmic rays are included, while they are essentially unaffected in the high mass case. Interestingly, we also see that the gaseous disks in the low mass halos appear to be "puffed up" by the 

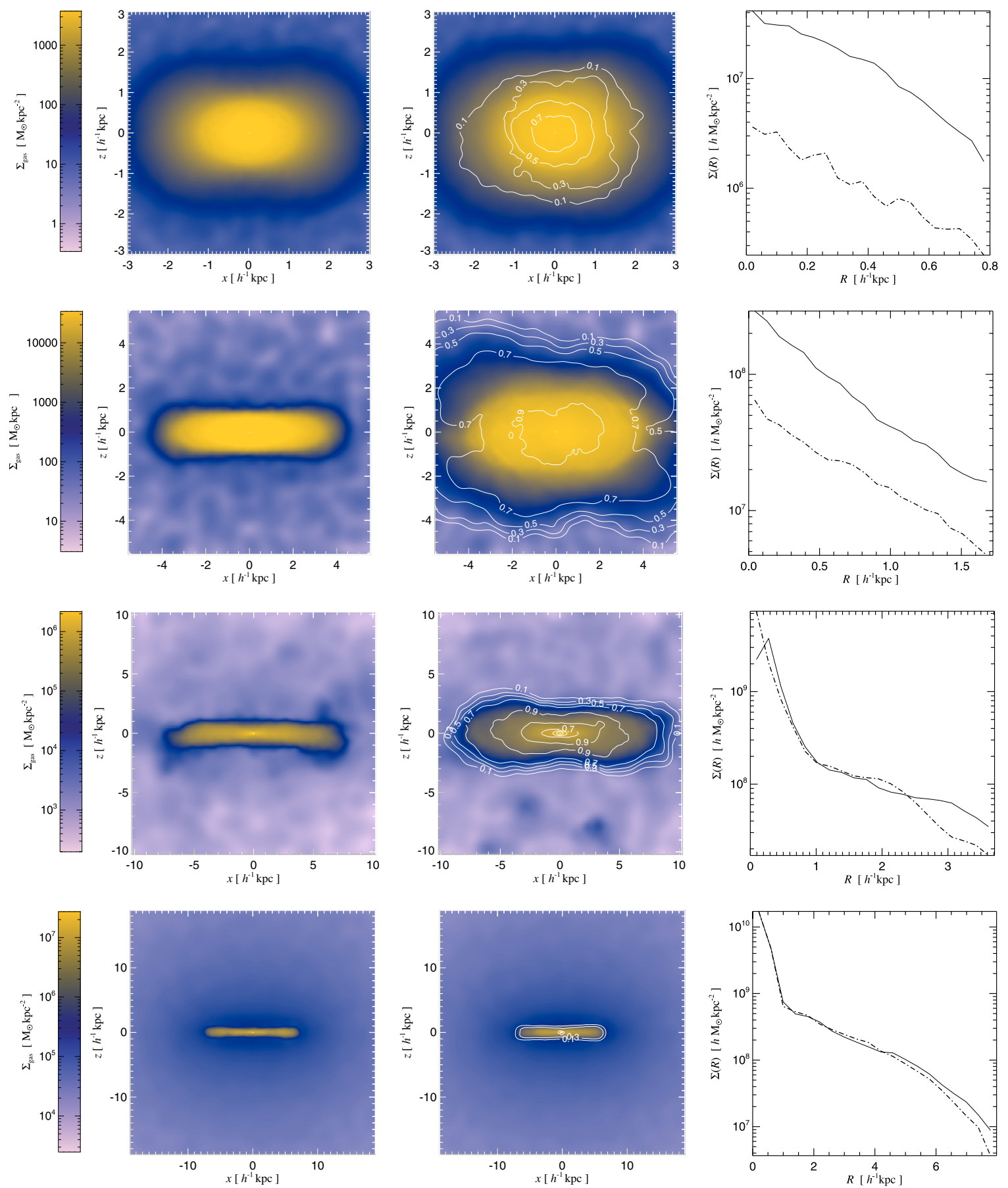

Fig. 13. Effect of cosmic ray feedback on star formation in simulations of isolated disk galaxy formation. Each row shows results for a different halo mass, for $M_{\text {halo }}=10^{9}, 10^{10}, 10^{11}$ and $10^{12} h^{-1} M_{\odot}$ from top to bottom. We compare the projected gas density fields at time $t=2.0$ Gyr of runs without cosmic ray feedback (left column) to that of runs with cosmic ray production by supernovae (middle column). The gas density field is colour-coded on a logarithmic scale. For the simulation with cosmic rays, we overplot contours that show the contribution of the projected cosmic ray energy density to the total projected energy density (i.e. thermal plus cosmic rays), with contour levels as indicated in the panels. Finally, the right column compares the azimuthally averaged stellar surface density profiles at time $t=2.0$ Gyr for these runs. Results for simulations without cosmic ray physics are shown with a solid line, those for simulations with CR feedback with a dot-dashed line.

additional pressure of the cosmic rays. It is remarkable that in the two low-mass systems there is substantial CR pressure found significantly above the star-forming regions, at densities much below the star formation threshold. This is despite the fact the acceleration of relativistic particles only occurs in star-forming regions of high density within the galactic disk in these simulations. Presumably, some of the CR-pressurized gas rises from the star-forming disk into the halo, a process that is suppressed by the stronger gravitational field in the high mass systems.

As a final analysis of our isolated disk simulations, we examine how well our simulation methodology for cosmic ray feedback converges when the numerical resolution is varied. To this end we repeat one of the simulations with cosmic ray feedback $\left(\zeta_{\mathrm{SN}}=0.1\right)$ of the $10^{11} h^{-1} M_{\odot}$ halo using both much lower and 


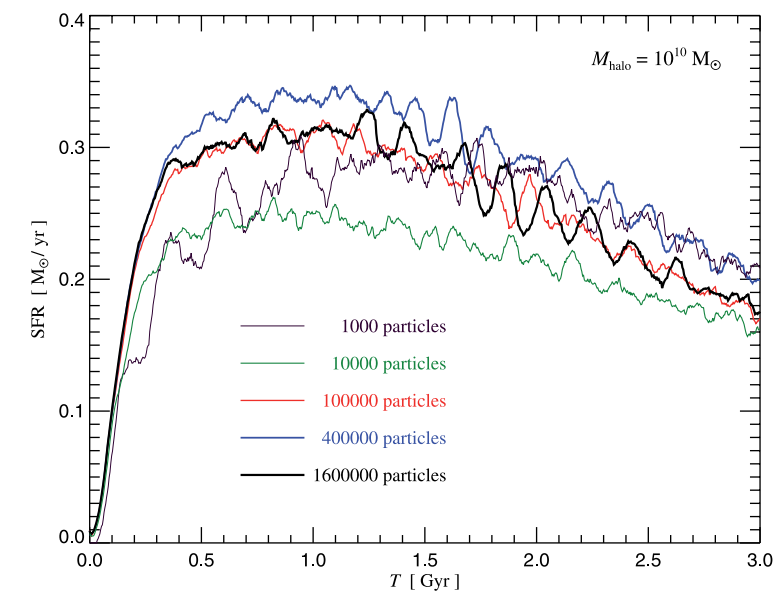

Fig. 14. Resolution study of the star formation rate during the formation of a galactic disk in a halo of mass $10^{10} h^{-1} M_{\odot}$, including production of CRs with an efficiency of $\zeta_{\mathrm{SN}}=0.1$. We compare results computed with $10^{3}, 10^{4}, 10^{5}, 4 \times 10^{5}$, and $1.6 \times 10^{6}$ gas particles, respectively.

much higher number of gas particles, namely $10^{3}, 4 \times 10^{5}$ and $1.6 \times 10^{6}$, respectively. In Fig. 14, we compare the resulting star formation rates. While there are some small fluctuations when the resolution is varied, there is clearly no systematic trend with resolution, and the results appear to be quite robust. In particular, the star formation rates for the simulations with $10^{5}$ and $1.6 \times$ $10^{6}$ particles are in very good agreement with each other despite a variation of the mass resolution by a factor of 16 . In fact, even the simulation with just $10^{3}$ produces a result that is still quite close to the high-resolution simulations. Note also that the oscillations are reproduced by all five resolutions, but they are not exactly in phase. Overall, this resolution test is very promising and suggests that the numerical model is well posed and can be applied to cosmological simulations where the first generation of galaxies is typically only poorly resolved. We can still expect meaningful results under these conditions.

\subsection{Cooling in isolated halos}

The comparison of the maximum cosmic ray cooling timescale with the thermal cooling time in the bottom panel of Fig. 5 has shown that for a relatively wide temperature range, the lifetime of CRs is larger than the thermal cooling time. In a composite gas with a substantial cosmic ray pressure component, this could potentially stabilize the gas temporarily and reduce the rate at which gas cools and accumulates at the bottom of the potential well of a halo. Also, models have been conjectured where the temperature structure of the intracluster medium, with its characteristic observed minimum of one-third of the ambient cluster temperature, could be explained by a strong CR component in the intracluster medium Cen (2005).

We here want to get an idea about the potential strength of this effect, and examine to this end a small set of toy simulations. To this end we consider a series of self-similar dark matter halos with a gas component that is initially in hydrostatic equilibrium, just as before in Sect. 5.1. In fact, we use the same initial conditions as before, except that we replace a fraction of the initial thermal pressure with cosmic ray pressure, keeping the total pressure constant. For definiteness, we choose a spectral cut-off of $q=1.685$ and a spectral index $\alpha=2.5$ for the initial CR population. We then evolve the halos forward in time, including radiative cooling processes for the thermal gas as well as cosmic ray

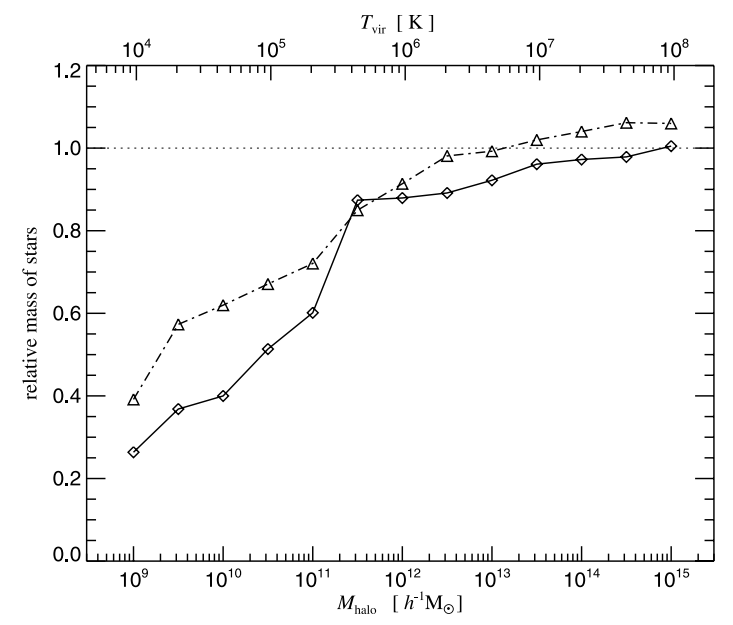

Fig. 15. Relative suppression of star formation in simulations of isolated halos when a fraction of 0.3 of the initial thermal pressure is replaced by a CR component of equal pressure. We show results as a function of halo virial mass for two different times after the simulations were started, for $t=1.0 \mathrm{Gyr}$ (solid line), and for $t=3.0 \mathrm{Gyr}$ (dot-dashed). For halos of low mass, the cosmic ray pressure contribution can delay the cooling in the halos.

loss processes, but we disregard any sources of new cosmic ray populations. We are interested in the question whether the cooling flows that develop in these halos are modified by the presence of the cosmic rays. Note that some studies have predicted cosmic ray pressure contributions of up to $\sim 50$ per cent in clusters of galaxies (Miniati et al. 2001; Ryu et al. 2003). These fiducial test simulations can give a first indication of the size of the change of the cooling rates if these claims are indeed realistic.

In Fig. 15, we show the results of these simulations as a function of halo mass, in the form of the integrated star formation rate relative to an equivalent simulation without initial CR population. The cumulative star formation activity can here be taken as a proxy for the integrated strength of the cooling flow in the halo. We see that the total star formation for cluster halos of mass $10^{15} h^{-1} M_{\odot}$ is essentially unchanged in the first $1-2 \mathrm{Gyr}$ of evolution, while at late times, it is even slightly increased. For systems of significantly lower mass, the star formation rates are however reduced in the CR case, by up to $\sim 40$ per cent. This can be qualitatively understood based on a comparison of the thermal radiative cooling time with the $\mathrm{CR}$ dissipative cooling time. As the lower panel of Fig. 5 has shown, the timescales are comparable at the virial temperature corresponding to the $10^{15} h^{-1} M_{\odot}$ halo, but are quite different for lower temperatures, where the radiative cooling is significantly faster. In fact, a naive comparison of these timescales would perhaps suggest an even stronger suppression of the cooling efficiency in systems of intermediate mass. In reality, the effect turns out to be much more moderate. This can be understood based on the softer equation of state of the CR component, combined with the fact that its cooling timescale typically declines faster than that of thermal gas when a composite gas is compressed. As a result, the ability of a CR pressure component to delay thermal collapse for a long time is quite limited, unless perhaps active sources for new populations of CR particles are present. Also, if metal line cooling is taken into account for the thermal gas, the relative influence of CRs may increase because the CR cooling time can then exceed the thermal cooling time at still higher temperatures, although the effect may be quite limited for very high gas temperatures, 
Table 1. Cosmological simulations of structure formation analysed in this study.

\begin{tabular}{|c|c|c|c|c|c|c|}
\hline Name & Physics & $\begin{array}{c} \\
{\left[h^{-1} \mathrm{Mpc}\right]}\end{array}$ & "Resolution & $\begin{array}{c}m_{\mathrm{DM}} \\
{\left[h^{-1} M_{\odot}\right]}\end{array}$ & $\begin{array}{c}m_{\text {gas }} \\
{\left[h^{-1} M_{\odot}\right]}\end{array}$ & $\begin{array}{c}\epsilon_{\text {grav }} \\
{\left[h^{-1} \mathrm{kpc}\right]}\end{array}$ \\
\hline S1 & non-radiative, no star formation (SF) & 100 & $2 \times 256^{3}$ & $4.30 \times 10^{9}$ & $6.62 \times 10^{8}$ & 15.0 \\
\hline S2 & $\begin{array}{l}\text { CR-injection at shocks with } \zeta_{\text {inj }}=0.5 \text {, } \\
\alpha_{\text {inj }}=2.5, \text { no star formation }\end{array}$ & 100 & $2 \times 256^{3}$ & $4.30 \times 10^{9}$ & $6.62 \times 10^{8}$ & 15.0 \\
\hline S3 & $\begin{array}{l}\text { Mach-number dependent CR injection at } \\
\text { shocks, no star formation }\end{array}$ & 100 & $2 \times 256^{3}$ & $4.30 \times 10^{9}$ & $6.62 \times 10^{8}$ & 15.0 \\
\hline D1 & $\begin{array}{l}\text { radiative cooling, star formation }(\mathrm{SF}) \text {, no } \\
\text { cosmic rays }\end{array}$ & 10 & $2 \times 256^{3}$ & $4.30 \times 10^{6}$ & $6.62 \times 10^{5}$ & 1.3 \\
\hline D2 & $\begin{array}{l}\text { radiative cooling, SF, CRs from supernovae } \\
(\mathrm{SNe})\end{array}$ & 10 & $2 \times 256^{3}$ & $4.30 \times 10^{6}$ & $6.62 \times 10^{5}$ & 1.3 \\
\hline D3 & $\begin{array}{l}\text { radiative cooling, SF, CRs from } \mathrm{SNe} \text { and } \\
\text { shocks (according to Mach-number) }\end{array}$ & 10 & $2 \times 256^{3}$ & $4.30 \times 10^{6}$ & $6.62 \times 10^{5}$ & 1.3 \\
\hline
\end{tabular}

where bremsstrahlung cooling dominates even in the presence of metals.

\section{Cosmological simulations}

Previous simulation work on the effects of cosmic rays on structure formation has not accounted for the dynamical effects due to cosmic ray pressure, i.e. the effectiveness of cosmic ray production has only been estimated passively. Interestingly though, these works suggested that the cosmic ray production may be quite efficient, with up to $\sim 50 \%$ of the pressure being due to CRs (Miniati et al. 2001; Ryu et al. 2003; Ryu \& Kang 2003, 2004). Here we present the first cosmological simulations of CR production that also account for the dynamical effects of cosmic rays. We first study the global efficiency of cosmic ray production at structure formation shocks. We then study the influence of cosmic ray feedback on star formation in cosmological simulations, and on possible modifications of the Lyman- $\alpha$ forest. Finally, we study the modification of the thermodynamic properties of the intracluster medium in high-resolution simulations of the formation of a cluster of galaxies. In all cosmological simulations were we included CRs, the cosmic ray population of the SPH particles was modelled with a constant slope of $\alpha=2.5$.

\subsection{Cosmic ray production in structure formation shocks}

In this subsection, we examine the efficiency of cosmic ray production at structure formation shock waves. To this end, we use simulations that include cosmic ray production injection at shocks and the cosmic ray loss processes (i.e. Coulomb cooling and hadronic losses), but we disregard radiative cooling and star formation. The cosmological model we have simulated is a concordance $\Lambda$ CDM model with parameters $\Lambda$ CDM model, with parameters $\Omega_{0}=0.3, \sigma_{8}=0.84$, baryon density $\Omega_{\mathrm{b}}=0.04$. We have picked a comoving box of side-length $L=100 \mathrm{~h}^{-1} \mathrm{Mpc}$, and simulated each of our models at two resolutions, with $2 \times 128^{3}$ and $2 \times 256^{3}$ particles, respectively. The results of the two resolutions are in good agreement with each other, so we restrict ourselves to reporting the results of the higher resolution runs with $2 \times 256^{3}$ particles in the following.

We compare three simulations that differ in the treatment of the cosmic ray physics. In our "full model", we account for shock injection using the Mach number estimator of our companion paper (Pfrommer et al. 2006) to determine the energy content and the slope of the proton populations accelerated at each shock front. This simulation hence represents our best estimate for the overall efficiency of the CR production process due to structure formation shocks. We contrast this simulation with a model where the CR injection has been artificially maximized by adopting a fixed efficiency $\zeta_{\text {inj }}=0.5$ and a fixed injection slope $\alpha_{\text {inj }}=2.5$ for all shocks. Note that this high efficiency is normally only reached as limiting case for high Mach number shocks, so that this model also allows us to assess the importance of the dependence of the shock injection efficiency on Mach number. Finally, we compare these two models with a reference simulation where no cosmic ray physics was included. This reference simulation is hence a standard non-radiative calculation where only shock-heating is included and the gas behaves adiabatically otherwise.

Table 1 lists the different cosmological simulations considered in this section, which are organized into two series of runs. Simulations $\mathrm{S} 1-\mathrm{S} 3$ focus on the effects of cosmic ray production at structure formation shocks, using a large cosmological box. Simulation S1 is a non-radiative reference run, while S2 includes $\mathrm{CR}$ production at structure formation shocks with a fixed high injection efficiency. In run S3, the injection efficiency is determined directly from the measured Mach numbers of the shocks. In the second series, D1-D3, we consider a much smaller volume at better resolution in order to study effects of CRs on the star formation rate in small galaxies. Simulation D1 is again a reference run with radiative cooling, star formation and ordinary supernova feedback, but no cosmic rays. Simulation D2 includes also CR injection by supernovae, while run D3 accounts for CRproduction both from supernovae and shocks. We note that we have also simulated lower resolution versions with $2 \times 128^{3}$ particles of the same runs to check for numerical convergence.

In Fig. 16, we compare the time evolution of the mean massweighted temperature of the full cosmic ray model to that in the ordinary non-radiative simulation. We also include a measurement of the mean energy in cosmic rays, converted to a fiducial temperature using the same factor that converts thermal energy per unit mass to temperature. Interestingly, at high redshift the cosmic ray energy content evolves nearly in parallel to the thermal energy, and both are roughly half what is obtained in the simulation without cosmic rays. Apparently, the thermalization of gas is dominated by strong shocks which reach the asymptotic injection efficiency of 50 percent. At late times, however, the CR energy does not grow as quickly as the thermal energy content any more, and the thermal energy in the CR simulation becomes closer to the thermal energy in the ordinary simulation.

These trends become more explicit when the energy content in CRs and in the thermal reservoir of the full CR simulation is 

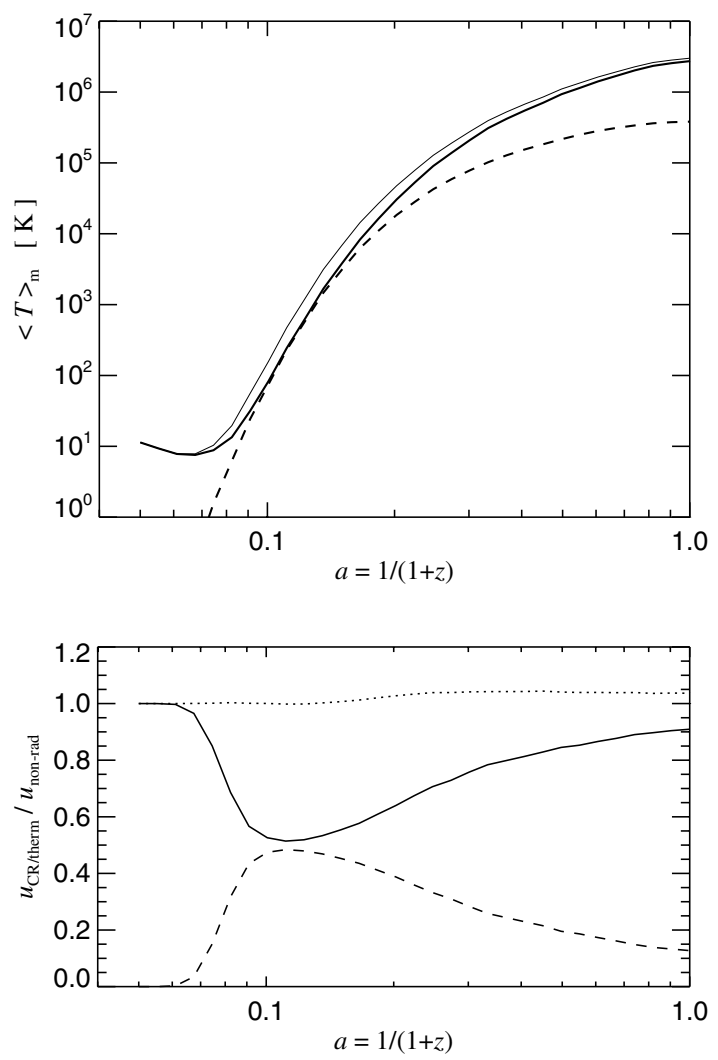

Fig. 16. Time evolution of the mean thermal energy and the cosmic ray energy content of the gas in non-radiative cosmological simulations. In the top panel, the solid thick line shows the mass-weighted temperature for a simulation where the efficiency of cosmic ray production at structure formation shocks is treated based on our on-the-fly Mach number estimator. The dashed line is the corresponding mean cosmic ray energy, which we here converted to a fiducial mean temperature by applying the same factor that converts thermal energy per unit mass to temperature. For comparison, the thin solid line shows the evolution of the mean mass-weighted temperature in an ordinary non-radiative simulation without cosmic ray physics. In the bottom panel, we show the ratio of the mean thermal energy in the cosmic ray case relative to the energy in the simulation without cosmic rays (solid line), while the dashed line is the corresponding ratio for the cosmic ray component. Finally, the dotted line gives the total energy in the cosmic ray simulation relative to the ordinary simulation without cosmic rays.

divided by the thermal energy content of the reference simulation, as shown in the bottom panel of Fig. 16. Around redshifts $z \sim 6-10$, the CR energy content nearly reaches the same value as the thermal energy in the full CR-model, and their sum is essentially equal to the thermal energy in the simulation without cosmic rays. Over time, the relative importance of the cosmic rays declines, however, and the thermal energy in the full CR model slowly climbs back to the value obtained in the nonradiative reference simulation. At the same time, the sum of cosmic ray and thermal energy obtained in the full model becomes a few percent higher at the end than that in the simulation without cosmic rays, despite the fact that the CR simulation loses some energy to radiation via the hadronic decay channels. Apparently, the simulation with cosmic rays extracts slightly more energy out of the gravitational potential wells of the dark matter. An explanation for this behaviour could derive from the fact that more energy needs to be invested into CRs to reach the same pressure compared with ordinary thermal gas. This should allow the gas in CR simulations to fall deeper into gravitational potential wells before it is stopped by shocks and pressure forces, such that more gravitational energy is liberated overall.

It is also interesting to ask how the relative importance of cosmic rays depends on gas density. This is addressed by Fig. 17, where we show the relative contribution of cosmic rays to the total gas pressure, as a function of baryonic overdensity, separately for different redshifts. We give results both for the simulation with Mach-number dependent shock injection (left panel), and for the one where we imposed a constant injection efficiency (right panel). In general, the importance of cosmic rays is largest for densities around the mean cosmic density, and declines towards higher densities. This is consistent with the expectation that the strongest shocks occur at low to moderate overdensities in the accretion regions around halos and filaments (Kang et al. 1996; Quilis et al. 1998; Miniati et al. 2000; Miniati 2002; Ryu et al. 2003; Pfrommer et al. 2006), and also with the growing importance of cosmic ray loss processes at high densities. Another interesting trend found in our simulation with Mach-number dependent injection (the "full model") is that cosmic rays are particularly important at high redshift, with a gradual decline towards lower redshift, suggesting that the mean dissipationweighted Mach number of shocks becomes lower at later times, as is indeed confirmed by studies of the cosmic Mach number distribution (Ryu et al. 2003; Pfrommer et al. 2006). Note that this trend is reversed in our fiducial simulation with a fixed shock injection efficiency, where at all overdensities the relative importance of CRs grows with cosmic time. This emphasizes that a correct accounting of the shock strengths is highly important even at a qualitative level to correctly model the evolution of the cosmic ray pressure distribution. We note that an implicit assumption we made in the above analysis is that weak magnetic fields are ubiquitous in the universe, even at low density. Whether they really exist and where they ultimately come from is an open question however.

In Fig. 18, we show the projected gas density field in a slice through the simulation box at $z=0$. To highlight the relative importance of cosmic rays, the panel on the right shows the ratio of the projected cosmic ray energy density to the projected thermal energy density. The relative importance of CRs is clearly highest in the volume-filling gas at low density. In the accretion regions around halos and filaments, the CR contribution is still comparatively large, but the high-density regions inside massive halos are avoided, in agreement with the results of Fig. 17. This raises the interesting question whether cosmic rays may perhaps modify the state of the intergalactic medium to the extent that the properties of Lyman- $\alpha$ absorption systems are modified. The latter arise primarily in gas that is largely unshocked, so that the effects might be weak even though the CR pressure contributions are predicted to be on average large exactly at overdensities of a few. We will come back to this question in Sect. 6.3.

Another interesting question is whether the bulk properties of halos are modified by the CR production at large-scale structure shocks. We are for example interested in the question whether the concentration of gas in halos is changed, which could manifest itself in a modification of the mean gas mass inside dark matter halos. To examine this question we determine halo catalogues for our simulations by means of the FOF algorithm with a standard linking-length of 0.2 , and measure the virial radii and masses by means of the spherical overdensity algorithm. In Fig. 19, we show the mean baryonic mass fraction in halos as a function of halo mass for the simulation with Machnumber dependent $\mathrm{CR}$ injection and for the run without cosmic ray physics. In both cases, the baryonic fraction within the virial radius lies slightly below the universal baryon fraction, reaching 

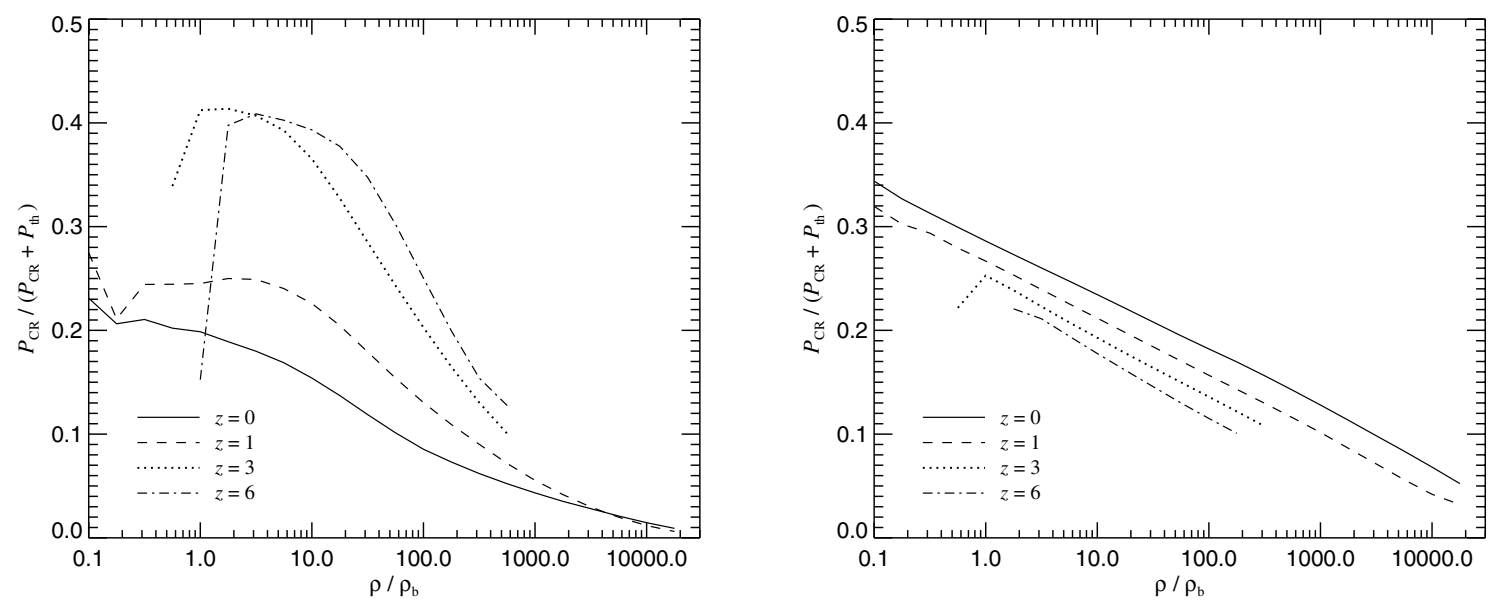

Fig. 17. Mean relative contribution of the cosmic ray pressure to the total pressure, as a function of gas overdensity in non-radiative cosmological simulations. We show measurements at epochs $z=0,1,3$, and 6 . The panel on the left gives our result for a simulation where the injection efficiency and slope of the injected cosmic ray spectrum are determined based on our on-the-fly Mach number estimation scheme. For comparison, the panel on the right shows the result for a simulation with a fixed injection efficiency $\zeta_{\text {lin }}=0.5$ and a soft spectral injection index of $\alpha_{\text {inj }}=2.9$. Clearly, the relative contribution of cosmic ray pressure becomes progressively smaller towards high densities. It is interesting that the trends with redshift are reversed in the two cases.
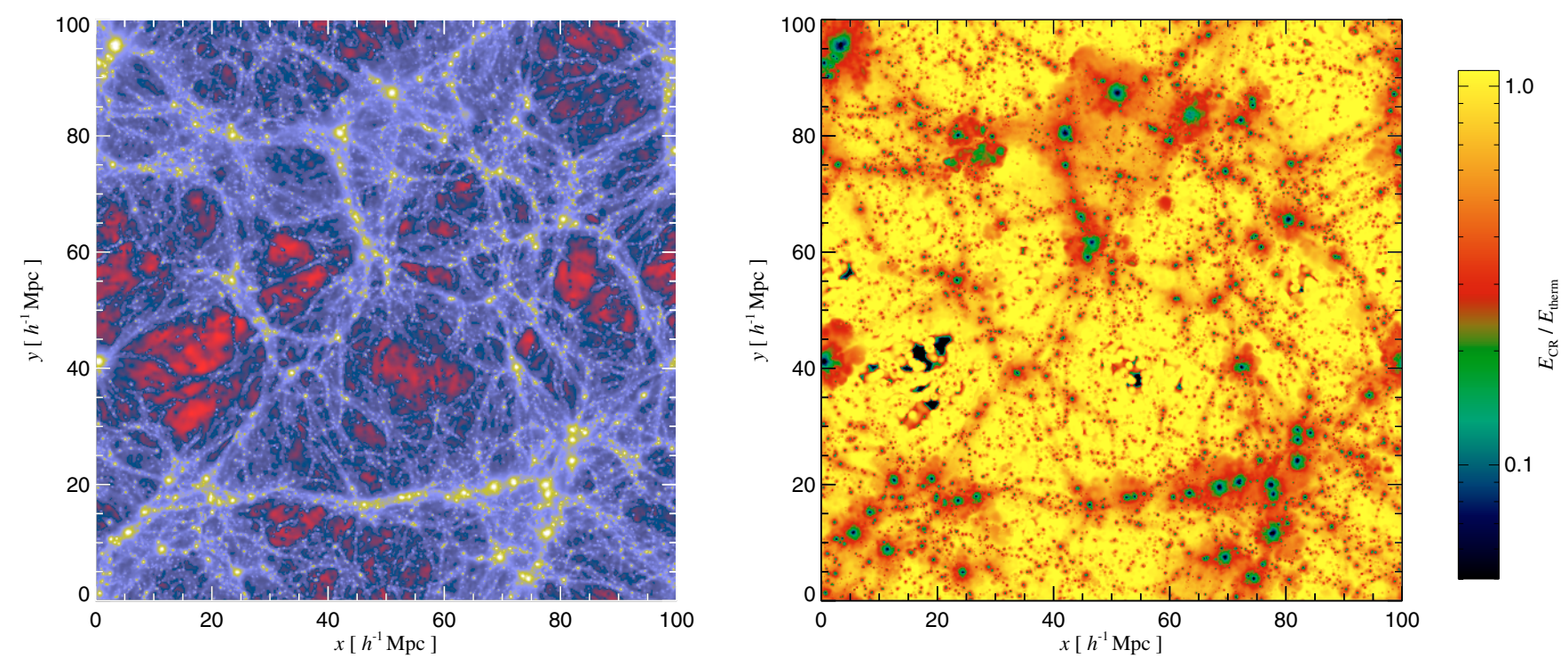

Fig. 18. Projected gas density field (left panel) in a slice of thickness $20 h^{-1} \mathrm{Mpc}$ through a non-radiative cosmological simulation at $z=0$. The simulation includes cosmic ray production at structure formation shocks using Mach-number dependent efficiencies based on an on-the-fly Mach number estimation scheme. The panel on the right shows the ratio of the projected cosmic ray energy density to the projected thermal energy density. We clearly see that the local contribution of cosmic rays is largest in voids. It is also still large in the accretion regions around halos and filaments, but is lower deep inside virialized objects.

$\sim 0.91-0.94$ of it, and for poorly resolved halos it drops a bit further. Such a depression of the universal baryon fraction is generally found in non-radiative SPH simulations (e.g. Frenk et al. 1999). However, a comparison of the two simulations shows that the halos in the run with CRs have systematically increased their baryonic fraction, albeit by only about $1-2$ per cent of the universal baryon fraction. This is consistent with expectations based on the higher compressibility of a composite gas with thermal and CR components.

Using the group catalogues, we can also measure the mean cosmic ray energy content inside the virial radius of halos. In Fig. 20, we show the ratio of cosmic ray to thermal energy as a function of halo mass. For the simulation with a fiducial shock injection efficiency of $\zeta_{\text {inj }}=0.5$ at all shocks, the ratio we obtain is $\sim 0.2$, independent of halo mass. Loss processes in the CR population and the shallower adiabatic index of the CR component make this value much smaller than expected in this case for the post-shock region of a single shock where one would expect $\sim 0.5$. In the simulation with Mach-number dependent injection of CRs, we find an interesting mass dependence where the ratio of CR-to-thermal energy drops from about 0.2 for $10^{12} h^{-1} M_{\odot}$ halos to $\sim 0.05$ for $10^{15} h^{-1} M_{\odot}$ halos. Apparently, for building up the thermal energy of clusters of galaxies, weaker shocks and adiabatic compression are comparatively more important than for galaxy-sized halos. We note that the value of $\sim 5-10 \%$ we predict here for the CR energy content due to structure formation shocks in clusters of galaxies is quite a bit lower than previous estimates Miniati et al. (2001). Future work is required 


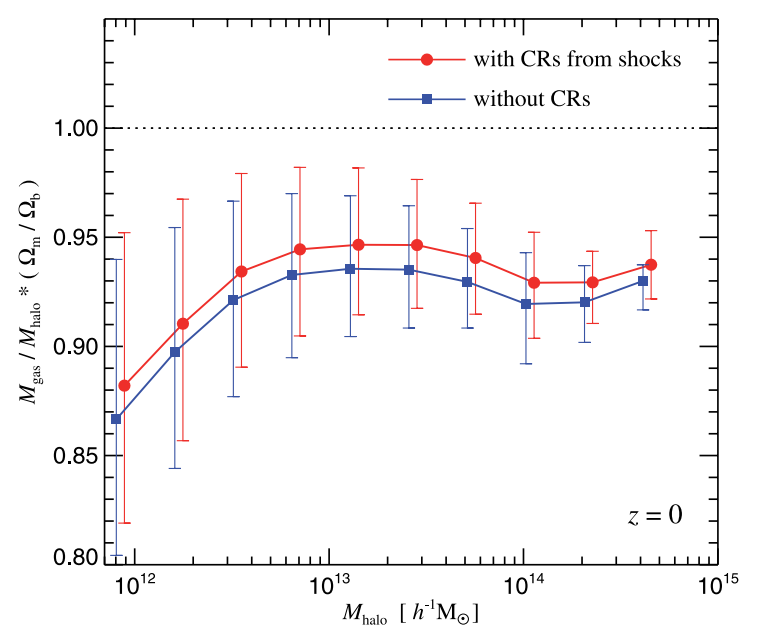

Fig. 19. Mean baryon fraction within the virial radius as a function of halo mass, normalized by the universal baryon fraction. We compare results for two non-radiative simulations, one with cosmic ray production by shocks, the other without cosmic ray physics. The bars show the $1 \sigma$ scatter among the halos in each bin. When cosmic rays are included, the compressibility of the gas in halos becomes larger, leading to a slight increase of the enclosed baryon fraction.

to further investigate the origin of this difference, which could partly be due to different parameterizations of the injection efficiency. However, it is in good agreement with CR constraints from gamma ray and radio observations (Pfrommer \& Enßlin 2003, 2004).

\subsection{Dwarf galaxy formation}

We now turn to studying the effects of cosmic ray feedback on galaxy formation in cosmological simulations. We have already found that small galaxies should be affected most. We hence expect small dwarf galaxies to be most susceptible to sizable effects of CR feedback from star formation. To reach a reasonably good mass resolution, we simulate periodic boxes of side-length $10 h^{-1} \mathrm{Mpc}$, using $2 \times 256^{3}$ particles. This gives a mass resolution of $6.62 \times 10^{5} h^{-1} M_{\odot}$ and $4.30 \times 10^{6} h^{-1} M_{\odot}$ in the gas and dark matter, respectively. We limit ourselves to evolving the simulations to a redshift of $z=3$, because at lower redshift the fundamental mode of the small simulation volume would start to evolve non-linearly, at which point the simulation as a whole could not be taken as representative for the universe any more. We are hence restricted to studying the high-redshift regime, but we expect that our results are indicative for the trends that would be seen in the dwarf galaxy population at lower redshifts as well, provided sufficiently well resolved simulations are available.

We have simulated the same initial conditions three times, varying the cosmic ray physics included. The first simulation is a reference run where we only included radiative cooling and star formation but no cosmic ray physics. The second simulation is a model where we also considered cosmic ray production by the supernovae associated with star formation, using an efficiency of $\zeta_{\text {SN }}=0.35$. Finally, our third simulation is a model where we in addition included cosmic ray production by structure formation shocks, using the Mach-number dependent efficiencies derived from our on-the-fly Mach number estimation scheme. The latter simulation hence represents our best estimate for the total effect of cosmic rays on dwarf galaxy formation.

In Fig. 21, we compare the cosmic star formation histories of the three simulations up to a redshift of $z \sim 2.9$. The

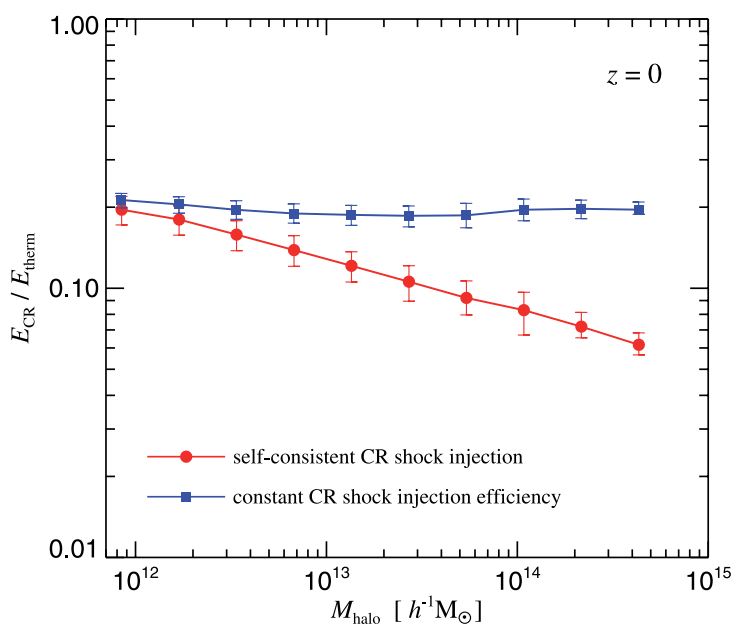

Fig. 20. Ratio of energy in cosmic rays to thermal energy within the virialized region of halos, shown as a function of halo mass. We compare results for two different non-radiative simulations, one treating the production of cosmic ray at shocks fronts using a Mach number estimator, the other invoking a constant injection efficiency. The bars give the $1 \sigma$ scatter among the halos in each bin. Interestingly, the Mach-number dependent injection scheme predicts a lower CR energy content in more massive systems. In contrast, a constant shock injection efficiency produced no significant trend of the CR energy content with halo mass.

incorporation of cosmic ray production by supernovae leads to a significant reduction of the high redshift star formation activity, but the shape of the star formation history, in particular its exponential rise, is not changed significantly. At these high redshifts, the star formation rate is dominated by small halos which are affected strongly by CR feedback, so this result is not unexpected given our previous findings. If CR production by structure formation shocks is included as well, the star formation is reduced further, although by only a small factor. This indicates that the cosmic ray pressure component injected into forming halos indeed tends to slightly slow down the cooling rates, consistent with the results we found for isolated halos. Towards redshift $z \sim 3$, the differences in the star formation rates become noticeably smaller however, suggesting that the low redshift star formation histories will differ at most by a small amount. Since the bulk of the star formation shifts to ever larger mass scales at low redshift (Springel \& Hernquist 2003b), this can be easily understood in terms of the smaller influence of CR feedback on large halos.

In order to make the effects of CR feedback on small halos more explicit, we determine halo catalogues in the simulations using a group finder. We are especially interested in the question how the efficiency of star formation is changed by the inclusion of cosmic rays as a function of halo mass. In Fig. 22, we show the total-to-stellar mass ratios of these groups as a function of halo mass, both for the simulation with CR production by supernovae, and for the simulation without cosmic ray feedback. The simulation where also CR production by shocks is included is quite similar on this plot to the simulation that only accounts for CR from supernovae, and is therefore not shown. The symbols show the mean total-to-stellar mass ratio in small logarithmic mass bins, while the bars indicate the scatter by marking the central $68 \%$ percentile of the distribution of individual halos. The results show clearly that the star formation is significantly reduced by CRs for low-mass halos, by factors of up to $\approx 10$ for host halo masses of $\sim 10^{10} M_{\odot} h^{-1}$ and below. On the other hand, the amount of stars produced in massive halos is hardly changed. 


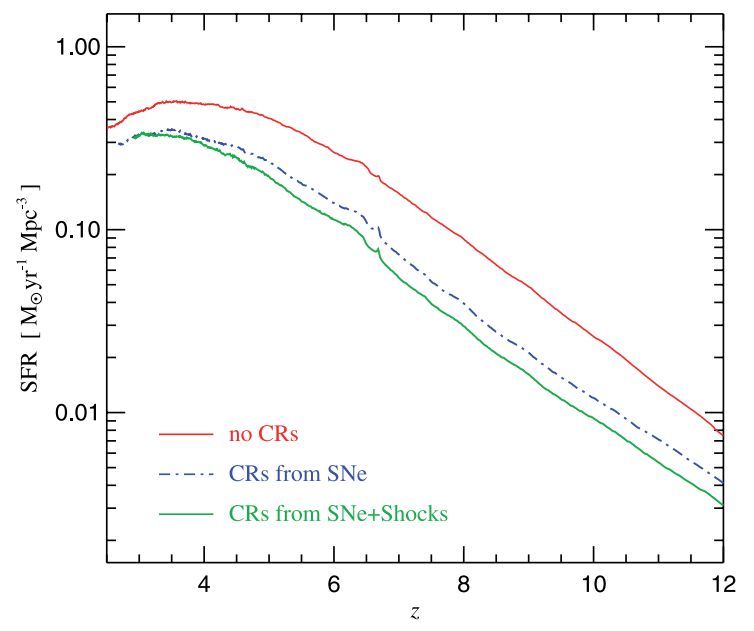

Fig. 21. Evolution of the cosmic star formation rate density in simulations of galaxy formation at high redshift. We compare results for three simulations that include different physics, a reference simulation without cosmic ray physics, a simulation with CR production by supernovae, and a third simulation which in addition accounts for CR acceleration at structure formation shocks with an efficiency that depends on the local Mach number.

It is particularly interesting that the effect of CRs manifests itself in a gradual rise of the total-to-stellar mass ratio towards lower masses. This can be interpreted as a prediction for a steeply rising "mass-to-light" ratio towards small halo masses, which is exactly what appears to be needed to explain the observed luminosity function of galaxies in the $\Lambda \mathrm{CDM}$ concordance model. The problem is here that the halo mass function increases steeply towards low mass scales. If the mass-to-light ratio is approximately constant for low masses, this leads to a steeply rising faint end of the galaxy luminosity function, in conflict with observations. However, a steeply rising mean mass-to-light ratio towards low mass halos could resolve this problem and provide a suitable "translation" between the halo mass function and the galaxy luminosity function.

We note that conditional luminosity function analysis of the 2 Degree Field Galaxy Redshift Survey (2dFGRS) has shown (van den Bosch et al. 2003) that there appears to be a minimum in the observed mass-to-light ratio of galaxies around a halo mass of $\approx 3 \times 10^{11} h^{-1} M_{\odot}$. This feature is reproduced surprisingly well in our simulations, although even with CR feedback included, the rise of the stellar mass to light ratio towards low masses appears to be not as sharp as required based on their analysis.

However, one needs to caution that the results of Fig. 22 cannot be naively translated into changes of the faint-end slope of the luminosity function, as seen when we directly compare the $K$-band luminosity functions at $z=3$. To determine those, we identify individual groups of stars as galaxies using a modification of the SUBFIND algorithm (Springel et al. 2001) for detecting bound substructures in halos. For each of the galaxies, we estimate magnitudes in standard observational band based on Bruzual \& Charlot (2003) population synthesis models. In Fig. 23, we compare the resulting rest-frame $K$-band luminosity functions at $z=3$ for the simulations with CR feedback by supernovae and the simulation without any cosmic ray physics. We see that both luminosity functions are well fit by Schechter functions, with faint-end slopes of $\alpha=1.15$ and $\alpha=1.10$, respectively, for the cases without and with $\mathrm{CR}$ feedback. We hence find that CRs only mildly reduce the faint-end slope

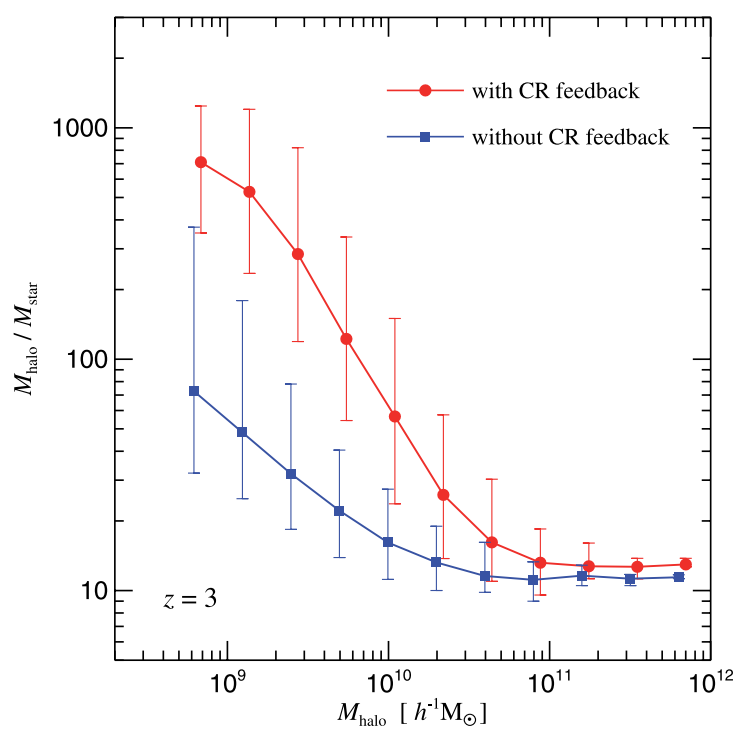

Fig. 22. Comparison of the averaged total mass-to-light-ratio within the virial radius of halos formed in two high-resolution cosmological simulations up to $z=3$. Both simulations follow radiative cooling and star formation, but one also includes CR-feedback in the form of cosmic production by supernovae, with an efficiency of $\zeta_{\mathrm{SN}}=0.35$ and an injection slope of $\alpha_{\mathrm{SN}}=2.4$. The bars indicate the scatter among halos in the logarithmic mass bins (68\% of the objects lie within the range marked by the bars). Clearly, for halo masses below $10^{11} h^{-1} M_{\odot}$, CR feedback progressively reduces the overall star formation efficiency in the halos.

despite their differential reduction of the star formation efficiency towards low mass scales. The result needs to be taken with a grain of salt though, as the faint-end slope could still be influenced by resolution effects in these simulations. A final assessment of the importance of CR feedback in shaping the faintend of the galaxy luminosity function needs therefore await future simulations with substantially increased resolution.

\subsection{Cosmic ray effects on the intergalactic medium}

As the Mach number distribution is dominated by strong shocks at high redshift, we expect that cosmic ray production is particularly efficient at early epochs and at the comparatively low densities where the strongest shocks occur, provided sufficient magnetization of the IGM existed to allow CR acceleration to operate. Also, the thermalization time scales of cosmic rays are quite long at low densities. Figure 17 has shown that the mean energy content of cosmic rays can reach a sizable fraction of the thermal energy content at around redshift $z \sim 3$, suggesting a potentially important influence on the intergalactic medium at this epoch. Note however that in computing the results of Fig. 17 we had neglected cosmic reionization, which will boost the thermal energy relative to the cosmic ray content. Also, large parts of the IGM at $z=3$, particularly those responsible for the absorption seen in the Lyman- $\alpha$ forest, consist largely of unshocked material. Whether the Lyman- $\alpha$ forest might show any trace of the influence of cosmic rays is therefore an interesting and open question.

To investigate this question further, we have computed Ly- $\alpha$ absorption spectra for the cosmological simulations with $10 \mathrm{~h}^{-1} \mathrm{Mpc}$ boxes analysed in the previous section. The two simulations we have picked both include radiative cooling, star formation, and heating by a spatially uniform UV background based on a slightly modified Haardt \& Madau (1996) model, 


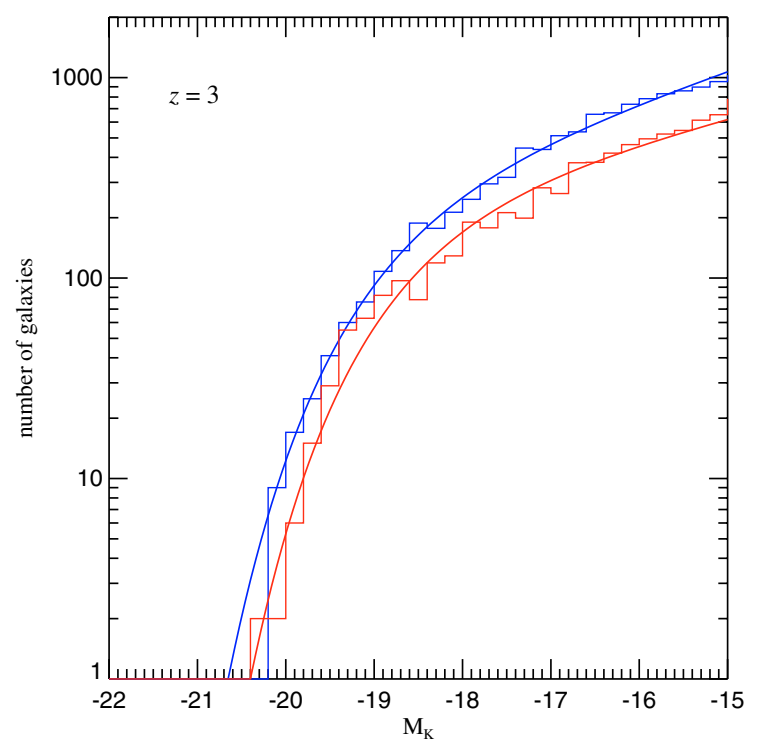

Fig. 23. The $K$-band galaxy luminosity functions at $z=3$ in two highresolution cosmological simulations. One of the simulations follows ordinary radiative cooling and star formation only (blue), the other additionally includes cosmic ray production by supernovae (red). The latter reduces the faint-end slope of the Schechter function fit (solid lines) to the data measured from the simulations (histograms). It is reduced from -1.15 to -1.10 in this case.

with reionization at redshift $z=6$. While one of the simulations did not account for any cosmic ray physics, the other included cosmic ray production by large-scale structure shocks and supernovae, as well as dissipative loss processes in the CR population.

For both simulations, we computed Lyman- $\alpha$ absorption spectra for 2048 lines of sights, along random directions parallel to the principal axes of the simulation boxes. By slightly adjusting the UV intensity, we have renormalized the spectra to the same mean transmission of $\langle\tau\rangle=0.68$. A direct comparison of the spectra along the same lines-of-sight through the two simulations shows essentially perfect agreement, with very small residuals. This already indicates that any systematic difference between the simulations must be quite subtle, if present. To objectively quantify this, we have computed the average 1-d flux power spectra for the two cases and compare them in Fig. 24. The top panel compares the two flux spectra directly with each other, and to observational data of McDonald et al. (2000). The results for the two simulations lie essentially on top of each other in this representation. The agreement with observational data is good, apart from a small excess of power on small scales, which can however be understood as a consequence of the too cool temperature of the IGM in our simulations compared with observations.

More interesting is perhaps an examination of the ratio of the flux power spectrum with cosmic rays to that without cosmic rays, as shown in the bottom panel of Fig. 24. While for largescale modes with $k<0.1 \mathrm{~km}^{-1} \mathrm{~s}$, no noticeable differences are seen, there is a $5-15 \%$ reduction of power in the wave-length range $0.1 \mathrm{~km}^{-1} \mathrm{~s}<k<0.7 \mathrm{~km}^{-1} \mathrm{~s}$, and at still smaller scales, the difference changes sign and turns into a growing excess of power in the CR simulation. These effects of CRs on the Ly- $\alpha$ therefore lie in a regime that is normally not used to constrain the matter power spectrum with Lyman- $\alpha$ forest data, at least in conservative treatments that focus on $k<0.03 \mathrm{~km}^{-1} \mathrm{~s}$ (Viel et al. 2004). In general we hence find that the effects on the Lyman- $\alpha$ forest are very small and subtle; the forest survives $\mathrm{CR}$ injection by
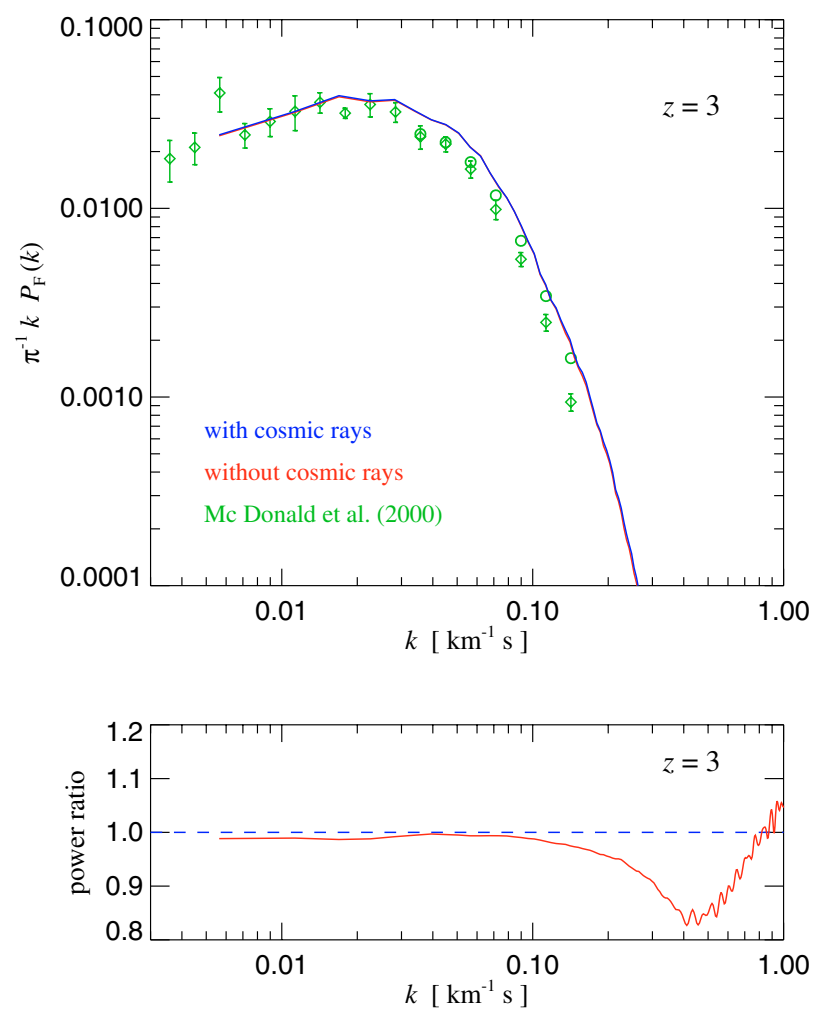

Fig. 24. Ly- $\alpha$ flux power spectrum (top) at $z=3$ in simulations with and without cosmic ray production in structure formation shocks. The results lie essentially on top of each other, and only by plotting their ratio (bottom panel), it is revealed that there are small differences. In the simulation with cosmic rays, the power is suppressed by up to $\sim 15 \%$ on scales $0.1 \mathrm{~km}^{-1} \mathrm{~s}<k<0.7 \mathrm{~km}^{-1} \mathrm{~s}$, while there is an excess on still smaller scales. However, on large scales $k<0.1 \mathrm{~km}^{-1} \mathrm{~s}$, which are the most relevant for determinations of the matter power spectrum from the Ly- $\alpha$ forest, the power spectrum is not changed by including CR physics. For comparison, we have also included observational data from McDonald et al. (2000) in the top panel (the open symbols are corrected by removing metal lines). A slightly warmer IGM in the simulations could account for the steeper thermal cut-off observed in the data.

large-scale structure shocks essentially unaltered, even though they contribute a sizable fraction to the mean energy content of the gas due to shock dissipation at densities at and around the mean density of the universe. Note that our simulations did not allow for a possible diffusion of CRs, but it seems unlikely that including this effect could change this conclusion.

\subsection{Formation of clusters of galaxies}

In this section, we study in more detail the influence of cosmic rays on individual halos formed in cosmological simulations. We focus on high-resolution "zoom" simulations of the formation of a massive cluster of galaxies. Such "zoom" simulations are resimulations of an object identified in a cosmological structure formation simulation with large box-size. Once the object of interest has been selected, its particles' are traced back through time to their origin in the unperturbed initial conditions. The Lagrangian region of the cluster thus identified is then populated with many more particles of lower mass, thereby increasing the local resolution, while in regions further away, the resolution is progressively degraded by using ever more massive particles. In this way, the computational effort can be concentrated in the 
object of interest, while at the same time its cosmological environment is still accounted for accurately during its formation.

We have computed 6 resimulations of the same cluster of galaxies, using different models for the physics of radiative cooling, star formation, and cosmic rays. The cluster has been selected from a set of zoomed cosmological initial conditions originally constructed by Dolag et al. (2004a) and has a virial mass of $\approx 10^{14} h^{-1} M_{\odot}$ at redshift $z=0$. The gas particle mass is $1.6 \times$ $10^{8} h^{-1} M_{\odot}$ in the high resolution region, implying that the cluster is resolved with roughly 300000 gas and 300000 dark matter particles within the virial radius. The gravitational softening length for the simulations was kept fixed in comoving units at redshifts $z \geq 5$, and then held constant in physical units at a value of $5 h^{-1} \mathrm{kpc}$ at lower redshifts.

Our 6 simulations fall into two groups of 3 each. In the first group, we have not included radiative cooling processes and star formation. Here we use a non-radiative ("adiabatic") simulation as a reference run, and compare it with two simulations that include cosmic ray production at large-scale structure formation shocks, one of them using the Mach-number dependent injection efficiency, and the other a fixed efficiency of $\zeta_{\text {inj }}=0.5$ with $\alpha_{\text {inj }}=2.5$ for all shocks. This set hence parallels the types of simulations analyzed in Sect. 6.1. In the second set of 3 simulations, we include radiative cooling and star formation. Again, we consider one reference simulation without any cosmic ray physics, and compare it with two simulations where cosmic rays are included. These two consist of one run where cosmic rays are only injected by supernovae associated with star formation (using $\zeta_{\mathrm{SN}}=0.35$, and $\alpha_{\mathrm{SN}}=2.4$ ), while the other also accounts for cosmic rays produced at shock waves. The second set of simulations hence corresponds to the types of simulations analyzed in Sect. 6.2. In all simulations with cosmic rays, we have assumed and index of $\alpha=2.5$ and included Coulomb cooling and hadronic losses for the CR populations.

In Fig. 25, we compare spherically averaged radial profiles of pressure, temperature, and gas density for the three non-radiative simulations. For the pressure, we show the thermal as well as the cosmic ray pressure for the two runs with cosmic ray physics. Interestingly, the cosmic ray pressure component is substantially below the thermal one, even in the fiducial case of a constant shock injection efficiency of $\zeta_{\text {inj }}=0.5$ for all shocks. However, in this case the thermal pressure is substantially elevated compared to the run without cosmic rays. This goes along with an increase of the gas density in the inner parts, and a reduction of the thermal temperature throughout the cluster volume. This is the expected behaviour based on the higher compressibility of the gas in this case.

However, the cosmic ray pressure in the simulation with a Mach-number dependent injection efficiency is substantially smaller, and even at the virial radius is at most $\sim 10$ percent of the thermal pressure, while for much of the inner parts, $r \leq 100 h^{-1} \mathrm{kpc}$, the cosmic ray pressure contribution drops to the percent level and below. Obviously, cosmic rays are not produced efficiently enough at shocks to fill much of the ICM with a dynamically significant cosmic ray pressure component, consistent with the results of Fig. 20. Consequently we find that in this case the profiles of gas density, temperature and thermal pressure are very similar to the corresponding results for the simulation without cosmic ray physics.

In Fig. 26, we show the equivalent results for the radial profiles for the 3 simulations that include radiative cooling and star formation. Compared to the non-radiative calculations, the ICM shows a markedly different structure. Due to the presence of a strong cooling flow, the temperature profile rises towards
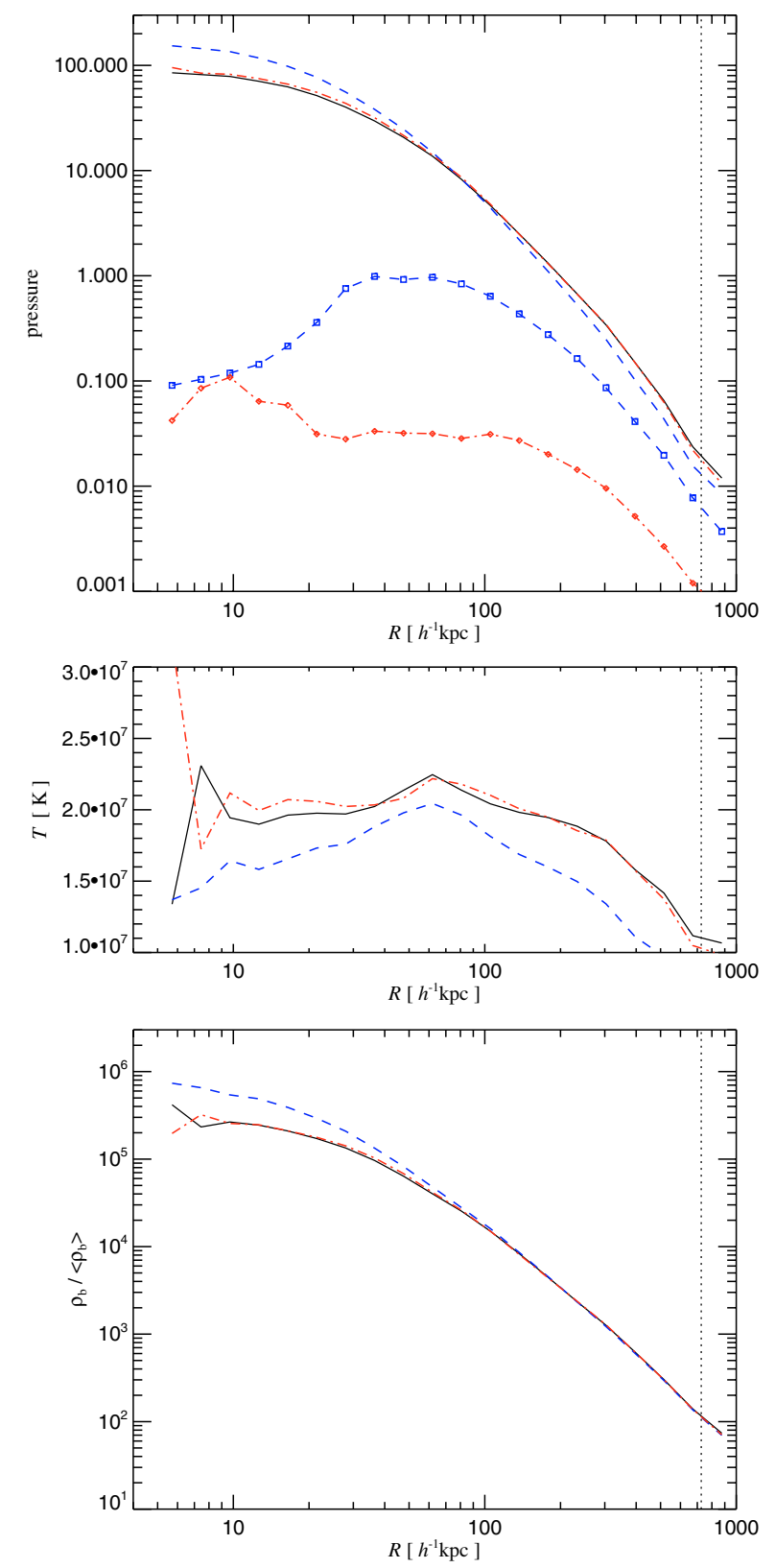

Fig. 25. Spherically averaged radial profiles of thermodynamic gas properties in three re-simulations of the same cluster of galaxies. All three simulations were not following radiative cooling and star formation, and the reference simulation shown with a solid line does not include any CR physics. However, the simulation shown with a dashed line accounted for CR production at structure formation shocks with a fixed efficiency $\left(\zeta_{\text {inj }}=0.5, \alpha_{\text {inj }}=2.9\right.$ ) while for the simulation shown with dot-dashed lines, the shock injection efficiency was determined with our Mach number estimation scheme. The panel on top compares the thermal pressure in the three simulations. For the two simulations with cosmic rays, we additionally plot the CR-pressure, marked with symbols. The panel in the middle compares the gas temperature, while the panel on the bottom shows the radial run of the gas density. The vertical dotted line marks the virial radius of the cluster.

the centre, until it eventually drops sharply at around $20 \mathrm{kpc}$ due to the onset of efficient cooling. The gas density has become significantly lower in the bulk of the cluster volume due to the large amount of gas that has cooled out, and correspondingly, the total pressure has fallen in much of the cluster volume. But there are also interesting differences in the simulations with 

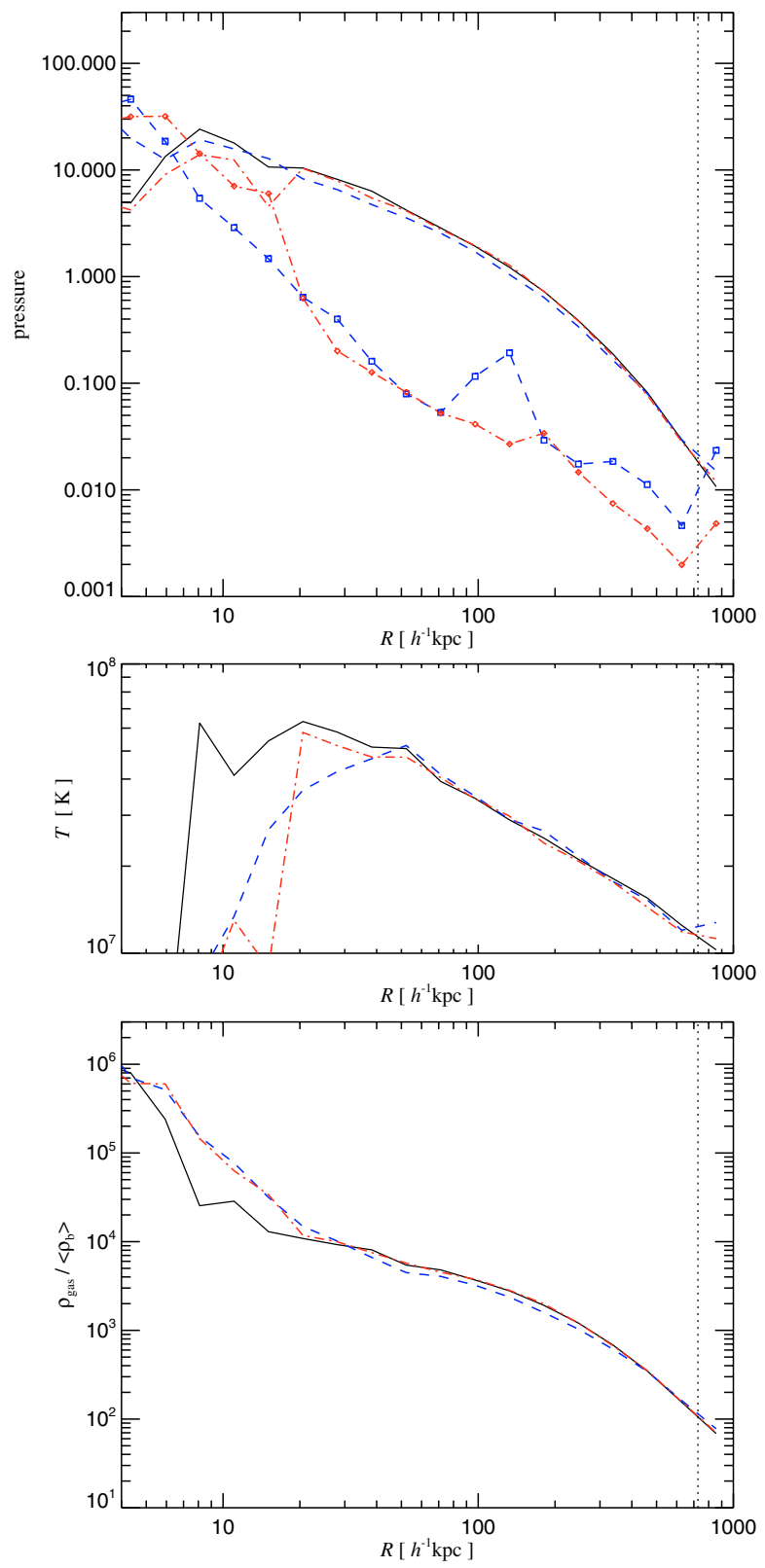

Fig. 26. Spherically averaged radial profiles of thermodynamic gas properties in three re-simulations of the same cluster of galaxies. All three simulations included radiative cooling of the gas, star formation and supernova feedback. The solid lines show the results of a reference simulation which did not include any cosmic ray physics. The other two simulations included $\mathrm{CR}$ production by supernovae, and the one shown with dot-dashed lines in addition accounted for CR injection at structure formation shocks, using Mach-number dependent efficiencies based on our Mach number estimation scheme. The panel on top compares the thermal pressure in the three simulations. For the two simulations with cosmic rays, we additionally plot the CR-pressure marked with symbols. The panel in the middle compares the gas temperature for the three cases, and the panel on the bottom shows the radial run of the gas density.

and without cosmic rays. Recall that both simulations included cosmic ray production by supernovae feedback, while only one of them accounted for cosmic rays by structure formation shocks. The CR pressure contribution in both simulations is quite similar through most of the cluster, at the level of a few percent of the thermal pressure. Also note that in the very inner parts, where the gas drops out through cooling, the cosmic ray

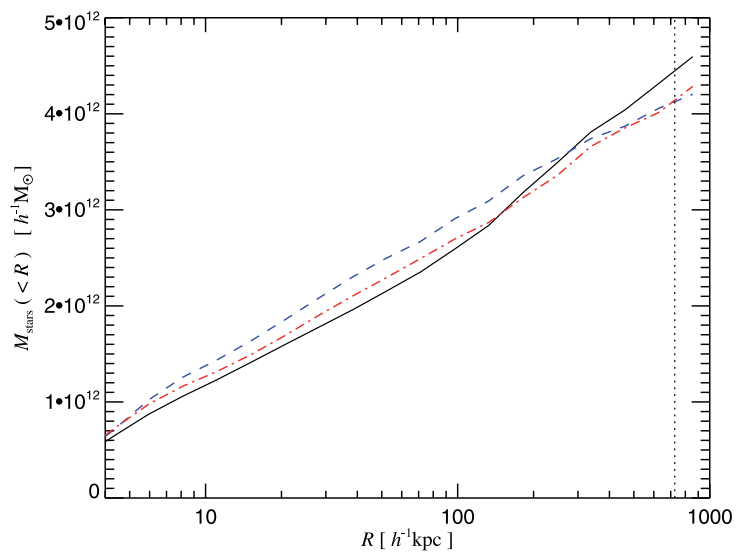

Fig. 27. Cumulative radial stellar mass profile in three re-simulations of the same cluster of galaxies. The simulations are the same ones also shown in Fig. 26. The solid line gives the result for a reference simulation without $\mathrm{CR}$ physics, the dashed line includes $\mathrm{CR}$ production by supernovae, and the dot-dashed line additionally accounts for CR injection at structure formation shocks. The vertical dotted line marks the virial radius of the cluster.

pressure rises sharply, even reaching and exceeding the thermal pressure. In this small region, the thermal pressure is dissipated more rapidly than the cosmic ray pressure.

Finally, in Fig. 27 we compare the cumulative stellar profile of the cluster in the three simulations with radiative cooling and star formation. While the total mass of stars formed within the virial radius has become smaller by the inclusion of cosmic ray feedback, the stellar mass in the central cluster galaxy has actually increased. The cluster cooling flow has therefore slightly increased in strength, consistent with the results we obtained for isolated halos of this mass range. On the other hand, the luminosity of the smaller galaxies orbiting within the cluster has become smaller, in line with our finding that small galaxies experience a reduction of their star formation activity. It is clear however that our results do not suggest that cosmic rays could provide a solution to the cooling flow problem in clusters of galaxies, at least not with the CR sources we have considered here. This conclusion could potentially change in interesting ways when $\mathrm{CR}$ production by AGN in clusters of galaxies is included as well (Churazov et al. 2001; Enßlin \& Vogt 2006).

\subsection{The influence of cosmic ray diffusion}

In all of our previous results, we have ignored the effects of cosmic ray diffusion, largely because of the uncertainty involved in constraining an appropriate diffusivity. However, diffusion could potentially be important in several environments, depending of course on the details of the magnetic field structure and the strength of the resulting diffusivity. While our present formalism implemented in the simulation code is capable of dealing with isotropic diffusion, in reality the diffusion is likely to be anisotropic, governed by the local magnetic field configuration. In principle, cosmological structure formation calculations with SPH are capable of following magneto-hydrodynamics (Dolag et al. 1999, 2005; Price \& Monaghan 2004, 2005), although this is presently still fraught with numerical and physical difficulties. We therefore postpone a detailed analysis of the influence of cosmic ray diffusion to future work. Here, we investigate instead a simple example, that gives a first illustration of the effects that can be expected. 

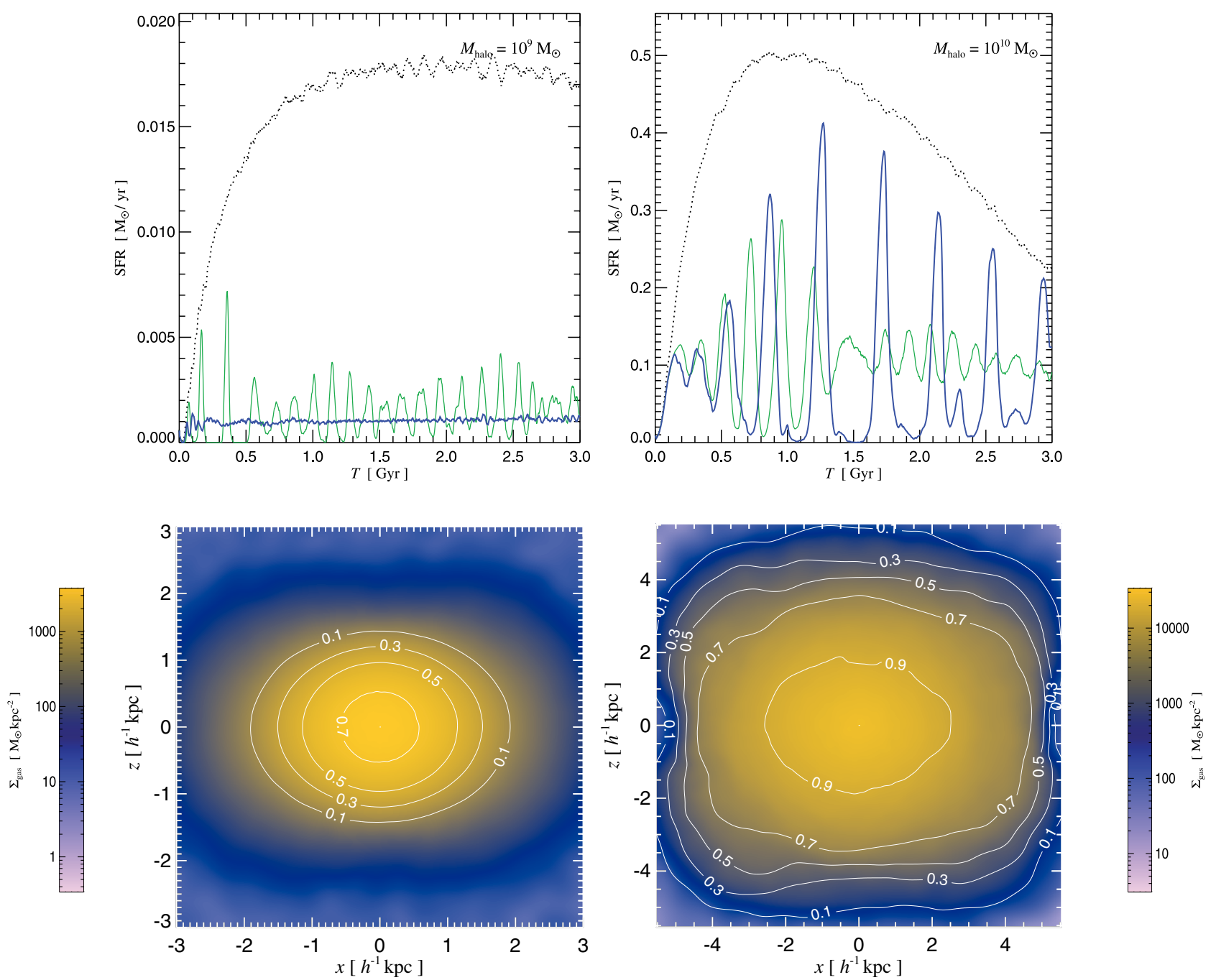

Fig. 28. Effects of cosmic ray diffusion on the star formation and the pressure distribution in isolated halos of mass $10^{9} h^{-1} M_{\odot}$ and $10^{10} h^{-1} M_{\odot}$. The panels on top compare the star formation rate when CR diffusion is included (thick blue line) to the case where it is neglected (thin green line). The dotted lines show the result when CR-production by supernovae is not included. In the bottom panels, we show projected gas density fields through the halos at time $t=2.0 \mathrm{Gyr}$, with contours overlaid that give the fractional contribution of the projected CR energy to the total projected energy. These panels correspond directly to equivalent maps shown in Fig. 13 for the case without CR diffusion.

To this end, we repeat our simulations of isolated disk galaxy formation with CR injection by supernovae, but this time with diffusion included. We use a parameterized diffusivity as described in Sect. 3, setting the values of the density and temperature scaling exponents to $n_{T}=1 / 6$ and $n_{\rho}=-1 / 2$, respectively, with a baseline diffusivity of $\sim 10 \mathrm{kpc}^{2} \mathrm{Gyr}^{-1}$ at the threshold for star formation, i.e. our diffusivity model is given by Eq. (54). The simulations we repeat are the ones considered in Sect. 5.1 with an injection efficiency of $\zeta_{\mathrm{SN}}=0.3$ for the production of CRs by supernovae.

In Fig. 28, we compare the resulting star formation rates for halos of mass $10^{9} h^{-1} M_{\odot}$ and $10^{10} h^{-1} \quad M_{\odot}$ as a function of time with the corresponding results without diffusion. Interestingly, the oscillations due to the unstable dynamics of a cosmic ray dominated ISM are substantially suppressed when diffusion is included. This effect is quite strong in the results for the $10^{9} h^{-1} M_{\odot}$ halo, where we now observe a nearly constant, quiescent star formation rate. For the $10^{10} h^{-1} M_{\odot}$ halo, the oscillations are only partially washed out and happen less frequently, but if they occur, they are stronger. Here the star formation rate of the galaxy develops a "bursty" character. Interestingly, diffusion actually reduces the integrated star formation still further; it drops by about $30 \%$ for the $10^{9} h^{-1} M_{\odot}$ halo, and by $21 \%$ for the $10^{10} h^{-1} M_{\odot}$ halo compared to the case without diffusion. Apparently, the cosmic rays that escape from the star-forming ISM into the less-dense gas in the halo are able to supply a partial pressure support that effectively reduces the rate at which gas cools.

The more extended and smoother spatial distribution of cosmic rays due to diffusion can also be appreciated in the bottom panels of Fig. 28, where we show projections of the gas density field with contours for the cosmic ray to thermal energy content overlaid. These panels directly correspond to the ones shown in Fig. 13 for the case without diffusion. However, for halos of mass $10^{11} h^{-1} M_{\odot}$ and more, diffusion with the strength considered here has no significant impact on the dynamics. The progressively larger size of more massive systems makes it ever more important for diffusion to efficiently transport CR energy across the system.

\section{Conclusions}

In this paper, we have presented the details of the first practical implementation of a simulation code capable of carrying 
out high-resolution simulations of cosmological structure formation with a treatment of cosmic ray physics. In particular, our method takes dynamical effects of pressure forces due to cosmic rays into account and therefore allows us to carry out studies of $\mathrm{CR}$ feedback in the context of galaxy formation. The underlying formalism for the treatment of cosmic rays is discussed in a companion paper (Enßlin et al. 2007) and forms a compromise between the complexity of cosmic ray physics and the requirements of computational efficiency. In particular, we use a simplified spectral representation in terms of power laws for the momentum distribution with a low momentum cut-off. This allows for a rather significant simplification at the prize of a moderate loss of accuracy. As Enßlin et al. (2007) have shown, the cosmic ray pressure is expected to be accurate to about 10 per cent in our model under steady state conditions. We argue that this is sufficiently accurate for our purposes given the other uncertainties and approximations involved.

Our formalism also makes use of an on-the-fly shock detection scheme for SPH developed in a second companion paper (Pfrommer et al. 2006). This method allows us to estimate Mach numbers in shocks captured during SPH simulations, such that we can use an appropriate efficiency for the CR injection at large-scale structure shock waves.

We have given an initial analysis of the principal effects of two sources of cosmic rays, namely CRs produced by supernova explosions, and by diffusive shock acceleration during structure formation. The loss processes we considered were Coloumb cooling and hadronic losses, which should be the most important ones. If desired, the modelling of these CR sink terms can be refined in the future within our methodology, and additional sources like cosmic rays from AGN can in principle be added as well.

There are several noteworthy results we obtained with our cosmic ray treatment in this study. First of all, our simulations of galaxy formation with cosmic ray production by supernovae indicate that cosmic ray pressure can play an important role in regulating star formation in small galaxies. Here we find a significant reduction of the star formation rate compared to the one without $\mathrm{CR}$ physics, provided cosmic ray production efficiencies of several tens of percent are assumed. In small galaxies, the mean densities reached in the ISM stay sufficiently low such that the CR pressure can exceed the effective pressure produced by the thermal supernova feedback. Once this occurs, the gas of the ISM is puffed up, quenching the star formation rate. Due to the comparatively long cosmic ray dissipation timescale, the CR-pressure survives for a sufficiently long time in these systems and develops a sizable impact on the star formation rate. In massive galaxies on the other hand, the ISM becomes so dense that the CR-pressure is unable to exceed the effective pressure predicted by the multi-phase model of Springel \& Hernquist (2003a), such that the star formation rates are not altered.

This effect on star formation also manifests itself in a reduction of the cosmic star formation rate density in cosmological simulations of galaxy formation. Here the SFR history is reduced at high redshift, where the bulk of star formation is dominated by small dwarf galaxies. As the star formation shifts to the scale of more massive halos towards low redshift, the reduction becomes progressively smaller. An interesting implication of the strong effect of CR feedback on small galaxies is that this reduces the faint-end slope of the resulting galaxy luminosity function, an area that continues to be a problematic issue for hydrodynamical simulations of galaxy formation within the $\Lambda$ CDM scenario. We have indeed detected this flattening, although with a weak strength overall. This difference compared with the stronger effect we found in our simulations of isolated galaxies could be due to resolution limitations in the cosmological runs, or perhaps be an effect of the idealized initial conditions used for the simulations of isolated galaxy formation. Another tantalizing effect of cosmic rays is that they help to keep gas in small galaxies more diffuse. This should in principle help to alleviate the "angular momentum problem", which describes the problem of an efficient angular momentum loss of gas to the dark matter caused by the early collapse of large amounts of gas in small halos. It is believed that this is a primary reason why present simulations generally fail to produce large spiral galaxies at low redshift. Cosmic rays physics might help to resolve this problem.

In simulations where we included cosmic ray injection by structure formation shocks, we find that they are produced efficiently at high redshift when structure formation ensues, driven by the high Mach-number shocks found at low to moderate overdensities. At low redshift on the other hand, most of the energy is thermalized in weaker shocks where the injection efficiency is much smaller. As a result, the mean energy content in cosmic rays can reach above $40 \%$ at redshifts $z \simeq 5$, but drops to $\sim 10 \%$ at low redshift. However, the relative energy content also shows a strong density dependence. It is highest at low to moderate overdensities and declines continously with density, such that deep inside halos, only comparatively little cosmic ray energy produced by shock waves survive. An important factor in this trend is the strong density dependence of cosmic ray loss processes, and the softer adiabatic index of CRs.

When non-radiative, fully cosmological simulations of the formation of galaxy clusters are considered, it is therefore not very surprising that we find that structure formation shocks can build up only a comparatively small cosmic ray pressure contribution inside clusters. Even at the virial radius this contribution reaches only about $10 \%$, but lies much lower in the inner parts of the cluster. When radiative cooling and cosmic ray production by supernovae are included, we find that supernovae can boost the mean CR energy density in the cluster, but the averaged contribution still stays at the percent level throughout the cluster volume, except at places where the gas rapidly cools. Here the $\mathrm{CR}$ pressure can temporarily dominate the pressure and delay the collapse briefly. Nevertheless, we find that CR production by supernovae and structure formation shocks is unable to reduce central cluster cooling flows. Instead, we in fact detect a slight increase of the cooling in the $10^{14} h^{-1} M_{\odot}$ cluster we have simulated. This can be understood as a result of the higher compressibility of the cluster gas in the cosmic ray simulations, leading to an increased central concentration of the gas and an elevated baryon fraction in the cluster, and thereby to higher cooling of gas in the centre overall. Note however that the currently discussed AGN feedback for cooling-flow quenching was not included in our simulations. On the other hand, the bulk of the cluster galaxies experience a reduction of their star formation rate when CR feedback is included, such that the cluster galaxy luminosity function is expected to develop a shallower faint-end slope.

Overall, our results suggest that cosmic ray physics is unlikely to drastically modify the physics of galaxy formation in the $\Lambda$ CDM model. However, cosmic rays help in areas where current model-building faces important problems, like for the faint-end slope of the galaxy luminosity function and the angular momentum problem. Our formalism for treating CRs in cosmological simulations should therefore be very valuable for future studies on the role of cosmic rays in cosmological structure formation. In particular, it would be highly interesting to examine the effects of CRs on the metal distribution of the universe, or on 
the dynamics of buoyant bubbles inflated by AGN in clusters of galaxies. It will also be important to provide an in-depth analysis of the role of cosmic ray diffusion in future work.

Acknowledgements. The simulations of this study were computed at the "Rechenzentrum der Max-Planck-Gesellschaft" (RZG), Garching. We are grateful to Nick Battaglia for useful discussions. We thank an anonymous referee for very constructive criticism that helped to significantly improve the presentation of the paper.

\section{References}

Abadi, M. G., Navarro, J. F., Steinmetz, M., \& Eke, V. R. 2003, ApJ, 591, 499

Aharonian, F., Akhperjanian, A. G., Bazer-Bachi, A. R., et al. 2006, Nature, 439, 695

Aharonian, F. A., Akhperjanian, A. G., Aye, K.-M., et al. 2004, Nature, 432, 75

Baugh, C. M., Cole, S., Frenk, C. S., \& Lacey, C. G. 1998, ApJ, 498, 504

Bell, A. R. 1978a, MNRAS, 182, 147

Bell, A. R. 1978b, MNRAS, 182, 443

Berezinskii, V. S., Bulanov, S. V., Dogiel, V. A., \& Ptuskin, V. S. 1990, Astrophysics of cosmic rays, Amsterdam: North-Holland, ed. V. L. Ginzburg Bieber, J. W., \& Matthaeus, W. H. 1997, ApJ, 485, 655

Brookshaw, L. 1985, Proc. Astron. Soc. Austr., 6, 207

Bruzual, G., \& Charlot, S. 2003, MNRAS, 344, 1000

Bullock, J. S., Dekel, A., Kolatt, T. S., et al. 2001, ApJ, 555, 240

Cen, R. 2005, ApJ, 620, 191

Churazov, E., Brüggen, M., Kaiser, C. R., Böhringer, H., \& Forman, W. 2001, ApJ, 554, 261

Cleary, P. W., \& Monaghan, J. J. 1999, J. Comput. Phys., 148, 227

Cole, S., Lacey, C. G., Baugh, C. M., \& Frenk, C. S. 2000, MNRAS, 319, 168

Cole, S., Percival, W. J., Peacock, J. A., et al. 2005, MNRAS, 362, 505

Croton, D. J., Springel, V., White, S. D. M., et al. 2006, MNRAS, 365, 11

Di Matteo, T., Springel, V., \& Hernquist, L. 2005, Nature, 433, 604

Dolag, K., Bartelmann, M., \& Lesch, H. 1999, A\&A, 348, 351

Dolag, K., Grasso, D., Springel, V., \& Tkachev, I. 2004a, J. Korean Astron. Soc., 37,427

Dolag, K., Jubelgas, M., Springel, V., Borgani, S., \& Rasia, E. 2004b, ApJ, 606, L97

Dolag K., Grasso D., Springel V., Tkachev I., 2005, J. Cosmol. Astro-Part. Phys., 1,9

Drury, L. O., Markiewicz, W. J., \& Voelk, H. J. 1989, A\&A, 225, 179

Duffy, P., Kirk, J. G., Gallant, Y. A., \& Dendy, R. O. 1995, A\&A, 302, L21

Enßlin, T. A. 2003, A\&A, 399, 409

Enßlin, T. A., \& Vogt, C. 2006, A\&A, 453, 447

Enßlin, T. A., Pfrommer, C., Springel, V., \& Jubelgas, M. 2007, A\&A, 473, 41

Fall, S. M., \& Efstathiou, G. 1980, MNRAS, 193, 189

Frenk, C. S., White, S. D. M., Bode, P., et al. 1999, ApJ, 525, 554

Giacalone, J., \& Jokipii, J. R. 1999, ApJ, 520, 204

Haardt, F., \& Madau, P. 1996, ApJ, 461, 20

Heitmann, K., Ricker, P. M., Warren, M. S., \& Habib, S. 2005, ApJS, 160, 28
Hudson, M. J., Smith, R. J., Lucey, J. R., \& Branchini, E. 2004, MNRAS, 352 , 61

Jones, T. W., \& Kang, H. 1993, ApJ, 402, 560

Jubelgas, M., Springel, V., \& Dolag, K. 2004, MNRAS, 351, 423

Kang H., Jones T. W., 1995, ApJ, 447, 944

Kang, H., \& Jones, T. W. 2006, Astropart. Phys., 25, 246

Kang, H., Ryu, D., \& Jones, T. W. 1996, ApJ, 456, 422

Kauffmann, G., White, S. D. M., \& Guiderdoni, B. 1993, MNRAS, 264, 201

Kennicutt Jr., R. C. 1989, ApJ, 344, 685

McDonald, P., Miralda-Escudé, J., Rauch, M., et al. 2000, ApJ, 543, 1

Miniati, F. 2001, Comp. Phys. Comm., 141, 17

Miniati, F. 2002, MNRAS, 337, 199

Miniati, F. 2003, MNRAS, 342, 1009

Miniati, F., Ryu, D., Kang, H., et al. 2000, ApJ, 542, 608

Miniati, F., Ryu, D., Kang, H., \& Jones, T. W. 2001, ApJ, 559, 59

Monaghan, J. J. 1992, ARA\&A, 30, 543

Murali, C., Katz, N., Hernquist, L., Weinberg, D. H., \& Davé, R. 2002, ApJ, 571, 1

Nagamine, K., Springel, V., Hernquist, L., \& Machacek, M. 2004, MNRAS, 350, 385

Narayan, R., \& Medvedev, M. V. 2001, ApJ, 562, L129

Navarro, J. F., Frenk, C. S., \& White, S. D. M. 1996, ApJ, 462, 563

Navarro, J. F., Hayashi, E., Power, C., et al. 2004, MNRAS, 349, 1039

Perlmutter, S., Aldering, G., Goldhaber, G., et al. 1999, ApJ, 517, 565

Pfrommer, C., \& Enßlin, T. A. 2003, A\&A, 407, L73

Pfrommer, C., \& Enßlin, T. A. 2004, A\&A, 413, 17

Pfrommer, C., Springel, V., Enßlin, T. A., \& Jubelgas, M. 2006, MNRAS, 367, 113

Power, C., Navarro, J. F., Jenkins, A., et al. 2003, MNRAS, 338, 14

Price, D. J., \& Monaghan, J. J. 2004, MNRAS, 348, 139

Price, D. J., \& Monaghan, J. J. 2005, MNRAS, 364, 384

Quilis, V., Ibanez, J. M. A., \& Saez, D. 1998, ApJ, 502, 518

Rechester, A., \& Rosenbluth, M. 1978, Phys. Rev. Lett., 40, 38

Riess, A. G., Filippenko, A. V., Challis, P., et al. 1998, AJ, 116, 1009

Ryu, D., \& Kang, H. 2003, J. Korean Astron. Soc., 36, 105

Ryu, D., \& Kang, H. 2004, J. Korean Astron. Soc., 37, 477

Ryu, D., Kang, H., Hallman, E., \& Jones, T. W. 2003, ApJ, 593, 599

Schlickeiser, R. 2002, Cosmic ray astrophysics, Astronomy and Astrophysics Library (Berlin: Springer)

Sijacki, D., \& Springel, V. 2006, MNRAS, 366, 397

Somerville, R. S., \& Primack, J. R. 1999, MNRAS, 310, 1087

Spergel, D. N., Verde, L., Peiris, H. V., et al. 2003, ApJS, 148, 175

Springel, V. 2005, MNRAS, 364, 1105

Springel, V., \& Hernquist, L. 2002, MNRAS, 333, 649

Springel, V., \& Hernquist, L. 2003a, MNRAS, 339, 289

Springel, V., \& Hernquist, L. 2003b, MNRAS, 339, 312

Springel, V., Yoshida, N., \& White, S. D. M. 2001, New Astron., 6, 79

Tegmark, M., Blanton, M. R., Strauss, M. A., et al. 2004, ApJ, 606, 702 van den Bosch, F. C., Yang, X., \& Mo, H. J. 2003, MNRAS, 340, 771

Viel, M., Haehnelt, M. G., \& Springel, V. 2004, MNRAS, 354, 684

White, S. D. M., \& Frenk, C. S. 1991, ApJ, 379, 52

Willick, J. A., Strauss, M. A., Dekel, A., \& Kolatt, T. 1997, ApJ, 486, 629

Yepes, G., Kates, R., Khokhlov, A., \& Klypin, A. 1997, MNRAS, 284, 235 